\title{
Designing ethical artifacts has resulted in creative design
}

\section{Empirical studies on the effect of an ethical design support tool}

\author{
Kaira Sekiguchi ${ }^{1} \cdot$ Koichi Hori ${ }^{2}$
}

Received: 11 December 2019 / Accepted: 30 July 2020 / Published online: 12 October 2020

(c) The Author(s) 2020

\begin{abstract}
Ethical aspects in engineering design have become increasingly important in recent years. A typical example is the recent rise of artificial intelligence (AI) ethics. This paper applies user studies of a design support tool to empirically verify that our ethical framework improves the creativity of an engineer's design activity. The design support tool provides an environment for the promotion of ethical design perspectives and description. The experiments focus on two functionalities: semiautomatic generation and scenario path recommendation. These functions are designed around the application of ethical design theory, which extends the hierarchical representation of artifacts. Doing this enables users to reconsider their themes at the highest level of the hierarchy and to apply a wider conceptual space of design solutions. For example, by reconsidering the positions of their research themes in the space of the representation field, users can semi-automatically edit them and identify focal areas. Using the scenario path recommendation, designers can update their research themes after considering the ethical impacts of those themes on stakeholders. Both functions are realized by exploiting a knowledge base of ethical and technological discourses. Finally, the ethical design theory is updated based on some unexpected results of our user studies with regards to the cyclic relationship among theory, tools (i.e., experimental equipment), and observed data. For example, temporal dimensional aspects were confirmed as important.
\end{abstract}

Keywords Creativity support · Design theory $\cdot$ Ethics $\cdot$ Ethical design $\cdot$ User study $\cdot$ Design science

\section{Introduction}

\subsection{Background and objectives}

The objective of this work is to empirically verify the creativity support effect of ethics to enhance creative design activities using our proposed design support tool. Our results show that engineers, business persons, and organizations, when integrating ethics into practical design, can use our

\section{Kaira Sekiguchi}

kaira@dfrome.com

Koichi Hori

hori@acm.org

1 Department of Aeronautics and Astronautics, Graduate School of Engineering, The University of Tokyo, 7-3-1 Hongo, Bunkyo-ku, Tokyo 113-8656, Japan

2 Department of Aeronautics and Astronautics, Graduate School of Engineering, The University of Tokyo, Tokyo, Japan theory and creativity support tool to contribute to a better society.

Recently, the importance of considering ethics in engineering has increased. A typical example is the rise in the discussion of artificial intelligence (AI) ethics. AI ethics is an area of applied ethics that investigates concerns about AI's impact on society. Research has offered guidelines, principles, and use cases for AI-related technicians and other stakeholders. Representative examples of AI ethics include IEEE's ethically aligned design (IEEE 2016, 2017, 2019), Asilomar AI Principles (Future of Life Institute 2017) and AI criteria of administrative organizations, such as (AI Network Society 2017a, b, c). Important characteristics of AI ethics is the integration of social-scale values, e.g., human dignity, rights, freedoms and cultural diversity to AI design, such as with Asilomar AI principles.

However, merely insisting on ethics integration is insufficient when promoting the practice of ethical design among engineers. Few AI engineers actively focus on AI ethics when they cultivate their functional themes, regardless of the 


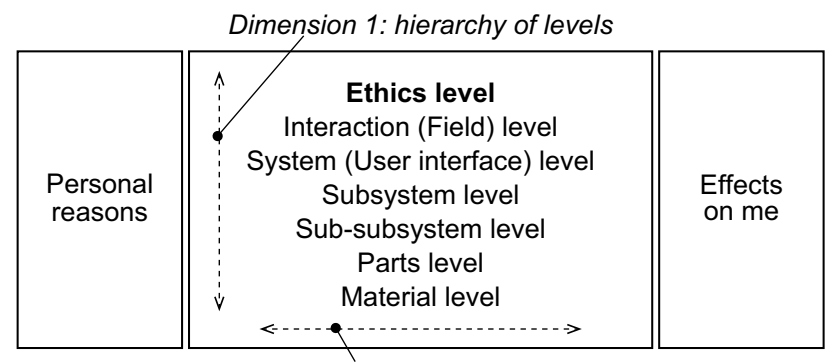

Dimension 2: comprehensiveness of ideas

Dimension 3: personal concerns

Fig. 1 Simplified version of the hierarchical and orthogonal representation of artifacts. Dimensions 1 and 2 comprise the hierarchical representation of artifacts, and dimension 3 corresponds to the orthogonal representation of personal concerns. See also Sekiguchi et al. (2010) and Sekiguchi and Hori (2018a, 2018b, 2019)

amount of discussion. Therefore, encouragement and good effects on such engineers should be necessary.

According to our ethical design theory (Sekiguchi and Hori 2018a, b, 2019), which will be detailed in Sect. 3, ethics comprises product constraints that could promote creative activities. Here, the theory deals with the impacts of integrating ethical values into an engineer's hierarchical representation of artifacts. We have positioned the level at which social ethical issues are to be dealt at the top of the engineering hierarchy (i.e., the "ethics level"). Ethical design theory also positions an orthogonal dimension to the hierarchy to express two types of personal concerns. The first that we apply includes personal reasons, such as designer motivations, problem awareness, interests, etc. The second applies effects on each stakeholder, such as incentives to the designer, choices by individuals, awards, etc. by connecting design aspects to consequences of the designer himself/herself or other stakeholders. These perspectives congeal into a model called "design from the ethics level." An overview of these perspectives is shown in Fig. 1. Details are found in Sect. 3.

Ethical design theory also describes a method that incorporates both ethical and technical perspectives. The basic idea is to describe changes caused by the designed artifacts and to associate them with the personal concerns and actual effects on stakeholders described above. A description of each change or each reason is called "item" and is positioned on the above-mentioned perspectives. These items are to be connected by "arrows" if they are considered to be causal. Example working descriptions include, "since it is a personal reason that I believe AI is a powerful tool, then I can generate a design that will change a car to be a self-driving vehicle," and "if a car is changed to be a self-driving vehicle, then a manufacturer will change to assume more responsibility towards car accidents." Using this method, design ideas will become traceable in a cause-and-effect chain which leads to the ethical issues connected by arrows. And the causality is to be a form of tree (or more precisely, a treelike ${ }^{1}$ description). This description method is what we call "design with discourse." Details are found in Sect. 3, too.

If it is true that ethics can affect creativity support, then it can inherently provide good personal reasons and effects for engineers. This hypothesis of the creativity support effect of ethics was presented by Sekiguchi and Hori (2018a), which provided use cases of our original design support tool: Dfrome. Dfrome is an organic and dynamic design support tool that promotes engineering practices of ethical design, especially ethical AI design (Sekiguchi and Hori 2018a, b, 2019). "Organic" implies that Dfrome handles complex relationships among different ethical and technical discourses, which can change dynamically. "Dynamic" implies that Dfrome adaptively adopts new issues and helps engineers think through their own contexts.

Two services of Dfrome are applied to this work: semiautomatic generation and scenario path recommendation. The first is a function that supports a designer's editing ideas by providing the tree-like forms described earlier. When drafting, the semi-automatic generation function automatically maps items from the tree-like description and connects them with arrows if causality appears to exist between them. Because this function is to be used during an interactive editorial task with the user, it is called "semi-automatic." Thus, the generated tree-like description placed on the perspectives will enable the designer to notice if and where his or her design ideas focuses on an ethical representation. The second is a function that provides scenario considerations regarding a designer's own context. The designer can choose that recommended scenarios be ethical or technical and similar or divergent. For example, a car manufacturer that produces a self-driving vehicle can use Dfrome to obtain ethical scenarios that organize the causal effects of the technology with regards to how it might change society, e.g., to realize greater freedoms for humans. Details are found in Sect. 4 .

We next organize hypotheses to be verified with user studies. The main hypothesis is about the existence of the creativity support effect of ethics. Then, there are some subhypotheses. For example, we can assume the mechanism of the effect: if a designer reconsiders a design decision because of ethical issues, he or she can obtain a wider conceptual space for design solutions that will allow him or her to develop even original design ideas. The former reconsideration corresponds to vertical operations in the hierarchical representation of artifacts (dimension 1 in Fig. 1). And the latter awareness of a wider conceptual space corresponds

\footnotetext{
1 This point to call the structure "tree-like" was also highlighted in a hearing of the user study. We adopted this subject's suggestion in the current paper.
} 
to the horizontal operations in it which corresponds to the comprehensiveness of design ideas (dimension 2 in Fig. 1). Additionally, the strong impact of the creativity support effect of ethics can be explained by its clear link to what is good for people and society. Details and the other hypotheses are found in Sect. 5.

We perform three types of user studies to empirically confirm the creativity support effect of ethics. In the first type, designers are required to consider the stakeholder impact that might be caused as a result of their research, development, and implementation. In the second type, designers are required to edit their research themes into a form defined by the ethical design theory in a short time. In the third type, designers are required to perform the second task over a longer period of time. Details are found in Sect. 6.

Our experimental environment exists on the Dfrome, where designers can interactively reconsider their ideas within a framework of ethical design theory and can interact with ethical and technological discourses accumulated in our knowledge base. With a series of user studies, we demonstrate that ethics can improve the creative activity of designers. This verification supports the introduction of ethics into engineering, which often proceeds by empirical approaches alone. Through its impact on innovation, the knowledge gained in from this research can also influence business practices. Details are found in Sects. 7 and 8.

\subsection{An example: Al-based privacy violation at a supermarket}

To present how ethics relate to engineering design and how they contribute to creative design, we introduce an imaginary example of an AI-based privacy violation that takes place at a fictitious supermarket. By developing this example, the introduction of ethical perspectives can be seen to cause a designer to reconsider the design purposes at higher conceptual levels and in wider conceptual space of design solutions. The contribution of this paper is that we explain and verify these kinds of phenomena using observed data.

We assume that a supermarket exists that considers privacy violations as non-problematic as long as the violations are not detected. This supermarket plans to earn as much money as possible to fulfill owner expectations. Thus, this supermarket aims to not only accurately suggest what a customer should buy, but to also sell their accumulated big data. Ultimately, when a person visits this supermarket, he or she is met with an automatic suggestion regarding what he or she should buy (e.g., via a coupon). The supermarket plans to adopt an AI system to accomplish this via face recognition technologies. By leveraging the identification data and associating them with a customer's purchase history, the system can also correlate information with internet databanks via social network services to build further predictive analyses.
The key problem with this scenario is the lack of consent. It is likely that many customers would be grateful to be met with accurate shopping predictions and associated benefits. However, a significant portion of the population would not be happy because of their lack of consent, and they would be faced with a dilemma regarding whether or not to continue shopping there. Even if the customer enjoys the capability, the supermarket's practice is still patently unethical.

Using our theory, we can redesign the supermarket AI system from an ethical background that, from the highest levels of ideation, can merge and balance the needs of privacy protection and sustainable development. For example, anonymisation could be applied, or, the supermarket could obtain overt consent from customers, such as through membership contracts. An advanced solution would be to provide two types of shopping baskets for the AI system: one that implies consent to share personal consumer information and another that does not, e.g., similar mechanism has been realized "for customers to indicate whether or not they want to speak to a salesperson" Springwise (2016), and we apply this mechanism to indicate such will to the AI system. It should also be possible to utilize the supermarket's personal data store (PDS) for privacy protection, which changes the controller of the personal information from the supermarket to the individual customers. An example of a PDS is the Personal Life Repository developed by Koiti Hasida (2004). Additionally, there may be a necessity to design a new mechanism to utilize PDS for the coupon service via face recognition, because it would be unique and proactive. Therefore, the supermarket could realize several new sustainable business alternatives, such as profiteering by obtaining a patent.

In this case, the ethical design theory is used to introduce ethics into the supermarket's AI business/engineering design by forcing the designers to reconsider their ideas at the highest levels of ethical purpose and to realize the wider conceptual space of design solutions. Using this theory, an extended version of the hierarchical engineering representation of artifacts could be used to address ethical artifacts. For example, privacy and sustainability issues could be considered at the ethics level. Customer experiences could be considered at the interaction level. Supermarket service could be considered at the system level, ... and facial recognition technologies could be considered at the parts level. The theory also has an axis for an orthogonal representation of personal concerns, which can be used to account for the personal reasons of the design, e.g., designers' and owners' ignorance of privacy protection, the motivation of fulfilling owner responsibilities, the utility of the AI system, etc. It can also be used to account for the individual effects of certain stakeholders, such that when a supermarket will earn a lot of money, some customers will want to utilize a coupon service, whereas others will not. 

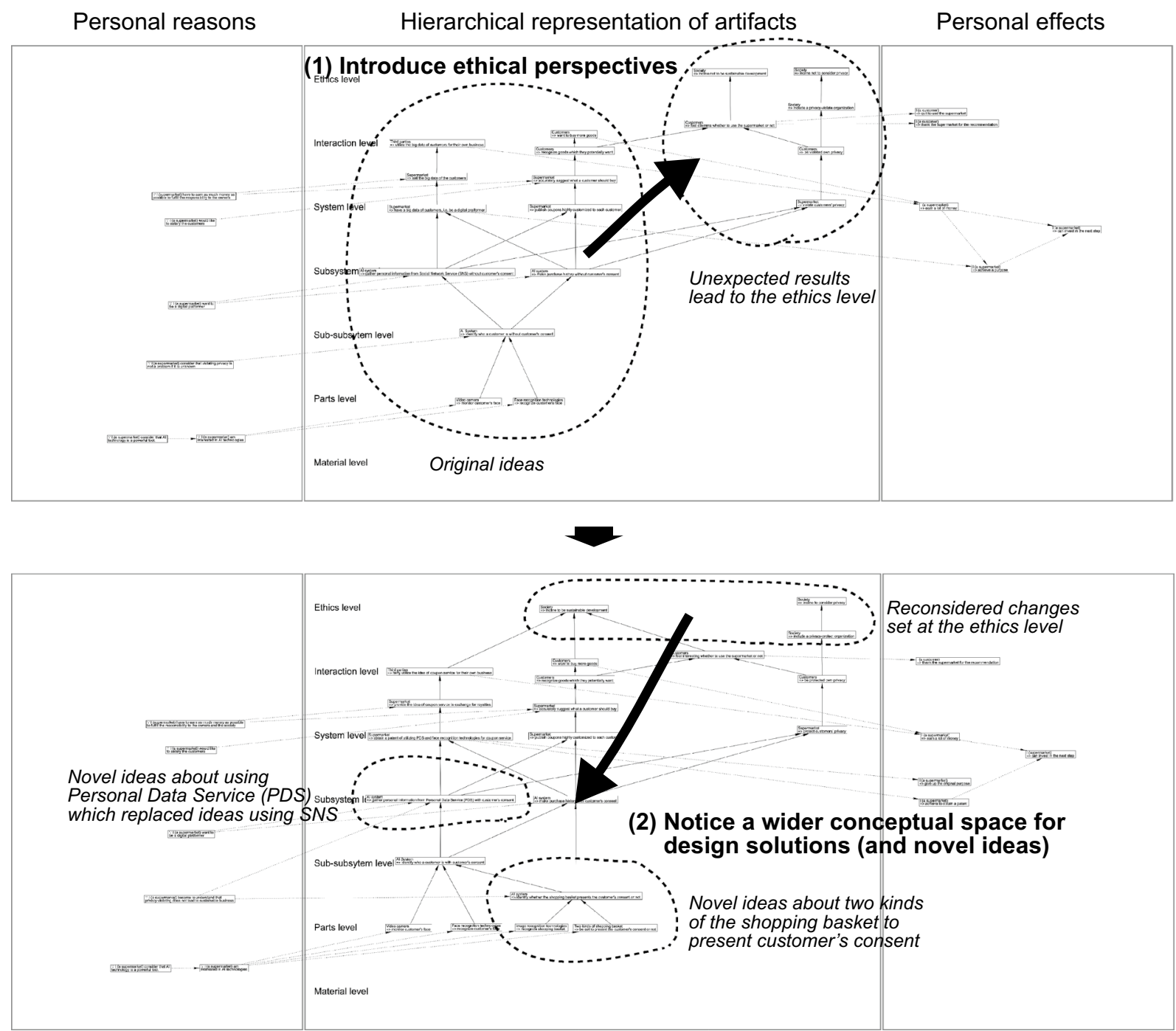

Fig. 2 Visualized image of the redesign of AI-based privacy violation at a supermarket

An illustration of this redesign is shown in Fig. 2. The design representation comprises three areas of the figure. The leftmost describes the personal reasons. The center reveals the hierarchical representation of artifacts. The rightmost describes personal effects. Then, the upper part Fig. 2 corresponds to the first element of the creativity support effect by ethics in which a designer introduces ethical perspectives. The lower part corresponds to the second element in which a designer notices a wider conceptual space for design solutions. These ideas coalesce into a tree-like form of items that describe changes connected by arrows that reveal causalities. Through this, we can achieve visual confirmation of the effects of ethics.

In Sect. 2, we introduce related work. In Sects. 3 and 4, we briefly review ethical design theory and Dfrome, respectively. We describe the hypotheses to be verified in Sect. 5. Then, we present the settings and results of the user studies in Sects. 6 and 7, respectively. A comprehensive discussion is presented in Sect. 8, and the paper concludes with a summary and suggestions for future work in Sect. 9. ${ }^{2}$

\section{Related work}

In this paper, our approach is the application of ethical design science, comprising the cyclic relationship among theory, tool (experimental equipment), and observed data where they intersect ethical design science and creativity support. Concretely, the theory is the ethical design theory,

\footnotetext{
2 Partial results of the work have been presented at ICED19, CRASSH2019 (poster) and Design Symposium 2019 (in Japanese).
} 
the tool is Dfrome, and the method of obtaining observed data is the user studies we conduct. Through this, we confirm the hypothesis that ethics support creativity, etc. Therefore, our work is positioned in relation to three disciplines upon which ethical design science is based: ethics, design science, and creativity support studies.

In recent years, the importance of introducing ethics into engineering, science, and technology has become quite extreme, especially with the rise of AI. Although many AI ethical discourses have taken place (IEEE 2016, 2017; AI Network Society 2017a, b, c; Future of Life Institute 2017), few engineers have applied these discourses to their own research and development. One probable cause of this lack of use is that ethical fields tend to rely on discussion rather than direct application. Verbeek (2011) provided a philosophical foundation for understanding technology as an ethical medium, assuming that technological ethical consideration should be a matter public attention. He further advocated for stakeholder engagement in public spaces to address the impact of technology on society. However, he did not emphasize the infusion of ethical practices by engineers into their everyday work. Ethical design theory provides a language that can be useful in public discourse. And the theory can also be directly applied to an engineer's backlog. Dfrome, in fact, expands the public space of ethical conversation to the online space of computer-aided engineering.

General ethics taught in ethical, legal, and social issues (ELSI) programs tend to imply that ethical considerations are barriers to innovation. For example, Mikami mentioned that science and technology researchers seldom engaged in ELSI-related activities, and that such activities were viewed by some researchers as unnecessary barriers to their own work. Furthermore, the exclusive reliance of such activities on ethics and law experts, "undermining the ' $S$ ' among E, L, and S of ELSI" Mikami (2019). Therefore, a mechanism that promotes the overt and voluntary ethical participation of engineers is necessary.

Then, design science further provides the way to explain what design is, namely the hierarchical representation of artifacts, as suggested by Simon (1996) and Yoshikawa $(1979,1981)$. However, these perspectives reach only the interaction level of the hierarchy and do not deal with ethics per se Nakakoji (2007). Then, by connecting ethics to design science, ethical design theory is considered capable of filling the gap between ethics and engineering (Sekiguchi and Hori 2018a, b, 2019). Concretely, we introduce a hierarchical representation of artifacts using ethical design theory, bringing it into a state of consistency with the engineering fields. Therefore, the ethical perspectives in ethical design theory are organically connected to engineering perspectives, suggesting that ethics could be incorporated by extending current engineering activities.
Approaches, such as value-sensitive design, also combine ethics and engineering design (Freedman et al. 2006; Miller et al. 2007; Spiekermann 2016; van der Poel 2015). However, they are not based on the hierarchical representation of artifacts. Therefore, it remains unfamiliar to engineers.

Another approach of design science is applied via design workshops at major design schools. For example, the d.school at Stanford University and the i.school at the University of Tokyo have adopted the thinking style of the IDEO Corp., an international design and consulting firm established in California, USA, in 1991 Kelly (2001). The style is based upon workshops and prototypes of humancentered design, but they have not yet provided a theoretical model to explain how ethics and engineering can be integrated.

Whitbeck (1998) utilized the similarities between ethical and design problems to explain ethics. She argued the pluralistic character of design solutions, wherein ethics was implied to be "ethics for professionals." In relation to ethical design theory, this sort of ethics relates to the orthogonal representation of personal concerns.

The creativity support effect has been generally reported in creativity support studies. However, problem-finding approaches have rarely been found (Wang and Nickerson 2017). A typical example of this general approach was described by Finke et al., who claimed that proper constraints could enhance creativity in design (Finke et al. 1992). However, they did not consider the ethical considerations under which each designer worked as applicable to the highest objectives of design. They argued that a general approach was meaningful and should differ from previous approaches with regards to particular tasks and problems. Our approach takes the middle position. Ours is more concrete than that of Finke et al., because we are determined to directly deal with ethical issues in design activities. However, our approach is still general in the sense that it can be applied to full range of artifacts, such as airplanes, spacecrafts, automobiles, commodities, buildings, social enterprises, etc. Because the ethical considerations relate to the highest purposes of design, their introduction makes our position more meaningful than the purely general position of Finke et al. Ethical design theory predicts that introducing ethics will enhance the creativity of design activities, because designers will be forced to reconsider the problem domains of each design. Finke et al. introduced several criteria for evaluating creative products, such as originality, practicality, sensibility, productivity, flexibility, etc. In this study, we focus on practicality and originality. If the scenario paths of design ideas could consistently be described from the lower to the highest levels in a hierarchical representation of artifacts, the design would become practical, because 
Fig. 3 An image of how ethical design theory can organize the three critiquing mechanisms, namely interpretive, specific and generic, and can add a new mechanism for reflection on realized artifacts which we call reflective (see Fischer et al. 1993 for the three critiquing mechanisms.)

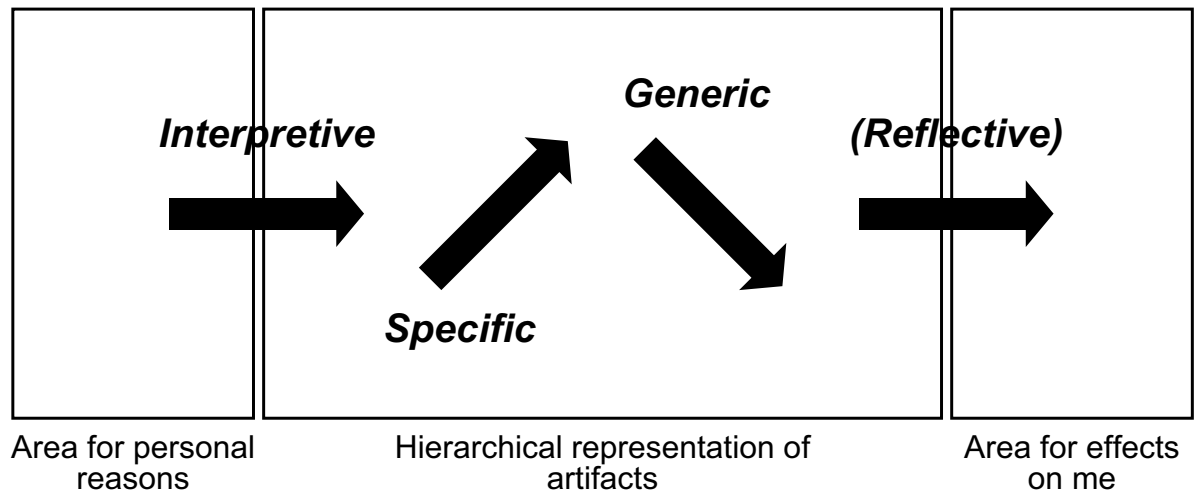

the means of realizing the artifact make better sense. When a designer can realize a wider conceptual space for design solutions, the originality of design ideas becomes more genuine. Thus, practicality, in this sense, corresponds to vertical operations within the hierarchy, and originality corresponds to its horizontal operations. From our user studies, we show that improved design ideas become more original, approaching the H-creativity condition of original thought (Boden 1991), because all subjects deal with original ideas during their studies or business operations.

Additionally, creativity-support studies have revealed the implementation features of creativity support tools and the actions of users applying the tools. Our creativity support tool, Dfrome, is an experimental tool that obtains observation data based on ethical design theory. The intention is to promote the practice of ethical design theory with engineers (Sekiguchi and Hori 2018a, b, 2019). The observed data must be suitable for checking and updating ethical design theory via trials that reflect real-world phenomena. This coherency is an important prerequisite of the cyclic relation of theory-experimental equipment-observed data in ethical design science. Therefore, another contribution of this paper is an updated ethical design theory based on real-world feedback. Via the given cyclic relationships, our outputs are more practical and quite valid.

Examples corresponding to the described cyclic relationship include the studies of Fischer et al. (1993). They utilized a successful human-critiquing paradigm to support the design of human-computer interaction capabilities. They classified three critiquing mechanisms: generic, specific, and interpretive. They additionally built creativity support tools and conducted user observations. From their work, we find that the generic critique mechanism corresponds to the generation of design solution. The specific mechanism corresponds to problem framing, and the interpretive mechanism corresponds to views. Moreover, they dealt with values such as good, safety, and efficiency, which, as a group, tends to be discussed from within ethical perspectives, although they seem to consider these values as characteristics of a system, i.e., at the system level. In their work, they dealt with a safe-kitchen system without considering social-scale values and whether a safe society would be a result of their system or even if it was desirable. Therefore the ethical issues were implicit in their work. They argued that "computer-based critiquing systems are most effective when they are embedded in domain-oriented design environments." Ethical design theory could be rendered more comprehensive if it were to combine the three critiquing mechanisms into one framework. A description of personal reasons corresponds to the interpretive mechanism. An upward consideration within the hierarchical representation of artifacts corresponds to the specific mechanism, and a downward consideration corresponds to the generic mechanism. Additionally, our improvement to the theory adds a new critiquing mechanism that corresponds to the description of effects on the designer (i.e., realized artifacts as depicted in Fig. 3). The reflective consideration can be described as imaginary when the design is being performed. It can also be practiced in the real world during social experimentation (Schinzinger and Martin 2000) or as an informationcycle loop (Yoshikawa 2012). In short, using ethical design theory, a designer can experience the various critical mechanisms in a more organic manner. Furthermore, ethical design theory demands that a designer be the most critical, because ethical values relate to the highest purposes of design. Thus, the creativity support effect is expected to be the strongest when a design is reconsidered for such purposes. Via user studies, this paper verifies the following point: criticism is still useful for supporting creative activities in relation to ethical perspectives.

An example that dealt with the given cyclic relationship was the Function-Behaviour-State (FBS) diagram of Umeda et al. (1997) and user studies that used the FBS modeler (Umeda et al. 1990). However, these work did not deal with ethical perspectives. Their definition of terms for FBS are as shown in Table 1.

Regarding the distinction between functions and Behaviours: functions are subjective, whereas behaviours can be objectively described when agents' views are selected (Umeda et al. 1997). Although these two concepts are similar to a certain extent, the grammar used to describe them is clearly different. The explanation of this point by themselves is as follows: 
Table 1 Definition of state, behaviour, function, and view (aspect) (Umeda et al. 1990)

\begin{tabular}{|c|c|}
\hline Term & Definition \\
\hline State & $\begin{array}{l}\text { W set of states } S \text { of an entity is described using internal states } S_{i} \text { and external states } S_{o} \text {. And } S_{i} \text { is described using entity, attribute and } \\
\text { relation }\end{array}$ \\
\hline Behaviour & "Aequential one and more changes of states." We consider a state which does not change for a while as a kind of behaviour \\
\hline Function & $\begin{array}{l}\text { "A description of behaviour abstracted by human thorough recognition of the behaviour in order to utilise it." ... it is repre- } \\
\text { sented in the form of " } t \text { to do something" }\end{array}$ \\
\hline View & $\begin{array}{l}\text { A view consists of a vocabulary of states, a vocabulary of behaviours, and a set of physical laws. Only after choosing a view, } \\
\text { we can describe states and behaviours }\end{array}$ \\
\hline
\end{tabular}

The boldface parts are the cited phrases

Fig. 4 An image of correspondence between ethical design theory and FBS diagram (see Umeda et al. 1990, 1997 for FBS diagram)

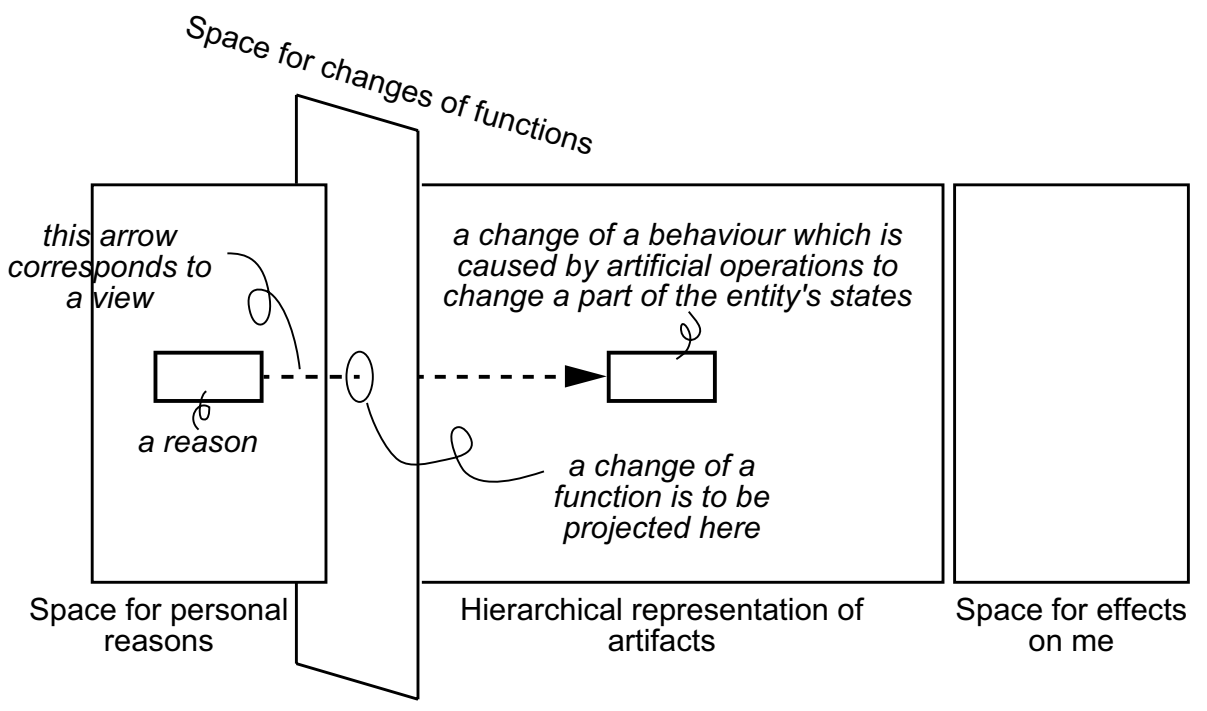

The vocabulary used for representing functions is often similar to that for behaviours. For example, support something for manufacturing it is a representation of a function, while $A$ is supporting $B$ is a representation of a behaviour of A. Umeda et al. (1990)

The problem suggested by Umeda et al. was that this definition of function is difficult to use. They described that their user studies showed that many designers felt it difficult to symbolize functions, especially those of unfixed entities. They then considered that describing behaviours was easier. In fact, they showed that describing behaviours was helpful to aiding the description of functions (Umeda et al. 1997). This situation can be clearly understood when we consider the correspondence between FBS diagrams and ethical design theory. First, the hierarchical representation of artifacts defined by the ethical design theory describes changes of behaviors that are caused by the artificial operations that change a part of the entity's state. Then, the ethical design theory establishes the hierarchical representation of artifacts to describe objective phenomena. For example, when building an FBS structure, Umeda et al. would say that $\mathrm{A}$ is supporting $\mathrm{B}$ as a representation of a behaviour.
Our description, on the other hand, says that $\mathrm{A}$ is changed to support B, A is changed to not support B, A is changed to support $\mathrm{C}$, etc. Thus, the space for changes of the functions of an FBS diagram is not our hierarchical representation. It stands between the space for personal reasons and the hierarchical representation of artifacts, as shown in Fig. 4. From this, ethical design theory demands that we describe views as personal reasons that are more subjective than the recognition of behaviors. The view of FBS diagram can be changed to incorporate arrows directed from personal reasons to the descriptions in the hierarchical representation of artifacts. Then, we can omit the FBS diagram's functions, because the subjective nature of the functional representation can now be expressed within the space of personal reasons, and the other items can be expressed as artifacts. Furthermore, we can add a space for describing the effects on the designer, so that we can provide a more comprehensive ethical design from theory. From our user studies, we show that the ethical design theory can solve the problem they suggested and that subjective matters of design can more easily be described with the ethical design theory than the FBS diagram. 
Dfrome connects standard discussions of ethics to technological developments and can be used to distribute the accumulated knowledge of specialists of different fields. In the past, science interpreters integrated humanities (e.g., ethics) with science and technology by promoting communications among participants. An early example was Murakami's intermediator (Murakami 2017). However, educating specialists and communicating with them is of limited practicality, and none of the existing approaches covered all disciplines. Dfrome resolves this limitation while providing a support tool for intermediators (i.e., interpreters) and engineers. Furthermore, interpreters tend to focus on discussions (Ema et al. 2017), just as with most ethical approaches, whereas our ethical design theory and Dfrome focuses on the design activity itself. This study thus provides a systematic explanation of our mechanism by which ethical considerations be used to directly support creativity for the participants.

\section{Ethical design theory}

Because the hypotheses for the user studies are derived from ethical design theory (Sekiguchi and Hori 2018a, b, 2019), we first provide an overview of the theory. Ethical design theory extends engineering design perspective to new areas while providing descriptive methods for handling both technical and ethical issues. Perspectives are design from the ethical level, and the descriptive methods are design with discourse.

\subsection{Design from the ethics level}

To promote engineers' adoption of ethical design, we must first, in two ways, redefine the perspective of engineers who use the hierarchical representation of artifacts. First, the ethics level, which expresses the social values, is placed at the apex of the hierarchical representation of artifacts. This representation (Fig. 1) is the basis of the systematic thinking proposed by Simon (1996) and Yoshikawa (1979, 1981). Here, ethics is a system of social values that includes human dignity, rights, freedoms, and cultural diversity, such as introduced by the Asilomar AI principles (Sekiguchi and Hori 2018a; Future of Life Institute 2017; Sekiguchi et al. 2010). A visualization of the new hierarchical representation of artifacts is depicted at the center of Fig. 1.

Next, a dimension of personal concerns is added orthogonally to the hierarchy dimension (Sekiguchi and Hori 2018a, b, 2019). This dimension describes the personal reasons of the designer, the changes of artifacts' behaviors in the hierarchical representation, and the effects of the designed artifacts on individuals and groups, in this order. Examples of personal reasons include awareness of issues, motivations, etc.,

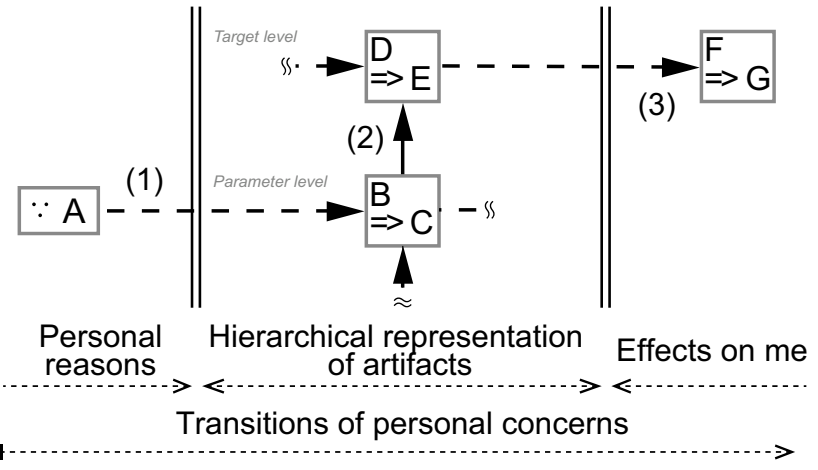

Fig. 5 Hierarchical structure and orthogonal grammar of "design with discourse" (Sekiguchi and Hori 2018a, b, 2019)

and examples of the effects on designers include incentives, rewards, etc. Ethical design theory enables designers to thus express their subjective aspects of their design ideas using a personal concerns representation. The visualized image of the space for describing personal reasons is seen at the left side of Fig. 1. The space for describing effects on the designer is seen at the right side.

\subsection{Design with discourse}

To systematically perform design from the ethics level, we also provide a description method. This method is called "design with discourse," which basically elicits descriptions of the changes introduced by the designed artifacts. The three description rules are listed below (Sekiguchi and Hori 2018a, b) and are visualized in Fig. 5. Each box in Fig. 5 is an item. Each arrow starts from a root item and ends at a target item. An expressed idea then becomes a tree-like description.

1. Since $\mathrm{A}$ is a personal reason, $\mathrm{I} /$ we generate a design that will change $\mathrm{B}$ to $\mathrm{C}$ in the hierarchical representation of artifacts.

2. If $\mathrm{B}$ is changed to $\mathrm{C}$ at the parameter level, then $\mathrm{D}$ will change to $\mathrm{E}$ at the target level in the hierarchical representation of artifacts.

3. If $\mathrm{D}$ is changed to $\mathrm{E}$ in the hierarchical representation of artifacts, then $\mathrm{F}$ will change to $\mathrm{G}$ as effects on me/us.

We then review the appearance of the tree-like description. Fig. 9 illustrates the AI-supported cashier case discussed by Sekiguchi and Hori (2018a), as referred to in (IEEE 2016, 2017; Oka and Morimoto 2015, 2016). In Fig. 6, an AI-based register system was designed to be installed in a bakery. When this system interacted with a cashier and a customer, it changed the job opportunities of the cashier and was expected to improve the freedom and equality of society via the customer. Additionally, it altered 
the costs afforded by the bakery and the shopping experience (benefits) that led to customer satisfaction.

Finally, we can understand that the propositions at levels higher than the target level in design will play the role of preconditions, referred to as Wittgenstein's "hinges" (Wittgenstein 1969) as discussed in (Sekiguchi et al. 2010; Sekiguchi and Hori 2019, 2018a). These are not to be doubted in design; they instead enable successful design at the target level and below. Thus, propositions at the ethical level almost always become a hinge, because the ethics level is the highest in the hierarchical representation of artifacts. One of our objectives is to clarify the existence of the ethics level and promote engineers' reconsiderations of design ideas from such highest objectives.

\section{The design support tool Dfrome and the experimental equipment}

Our user studies evaluated the design support effect of ethics. This tool, called Dfrome, was set as the experimental environment. Therefore, the Dfrome is reviewed in this section.

\subsection{Overview of Dfrome}

Dfrome was first implemented to help AI engineers practice the design of ethical AI. Dfrome comprises four main components: an editor for editing tree-like ideas; a browser for viewing the accumulated tree-like data of ethics and technologies; a knowledge base for accumulating ideas into a cloud environment; and an investigation engine that supports idea editing, recommending scenario paths, and performing other functions using natural language processing. The latest version of Dfrome is overviewed in Fig. 10 (Sekiguchi and Hori 2018b). For the user studies presented later in this paper, semi-automatic generation functions were added to the investigation engine. We next describe details of Dfrome's components.

\subsection{Editing ideas on Dfrome's editor}

A user edits his or her ideas using the Dfrome editor. The interactivity of the editing function is important for intuitive expression by users. In the user studies, users are tasked to use two advanced services of Dfrome: one with editorial support via semi-automatic generations (a new service), and another with scenario path recommendation. ${ }^{3}$

\footnotetext{
3 The use of Dfrome's editor is described at https://www.dfrom e.com/how_to_editor. Similar information is given in Dfrome's manual for the user studies and was explained to the subjects of the user studies.
}

\subsubsection{Editorial support by semi-automatic generation}

Editorial support on Dfrome is provided as a draft for expressing ideas in tree-like form. A user can obtain and edit a draft to better express and position design ideas. Because this service requires input and re-editing by the user, it is called semi-automatic. An example of the editing process on Dfrome is demonstrated in Fig. 11. Here, a user can input an abstract of his or her design idea and update it by obtaining a semi-automatically generated tree-like description, which can be re-edited, as shown in Fig. 11.

Figure 12 shows an actual example of semi-automatic generation. The input text is the abstract of a paper (Sekiguchi and Hori 2019). Here, we express the significance of verifying generative ethics. Although the generated tree-like description was fairly satisfactory, it could be improved by editing. For example, we could move item (d) from the subsystem level to the system level. Thus, the editor can be used to discern points of difference between the calculated results and what the user explicitly or implicitly has in mind. Doing this, users can reconsider the structure of their ideas and where they should focus their considerations.

To realize this capability, two kinds of semi-automatic generation functions are provided. The first function maps the items of the design ideas, and the second connects the related items with arrows. The calculation processes of these semi-automatic generation functions are overviewed in Sect. 4.5.

\subsubsection{Scenario path recommendation of Dfrome's editor}

The full version of the scenario path recommendation is provided in Dfrome's editor. Using this version, users can confirm related scenario paths along forward or backward causality arrows. Users are given the other option: confirmation of paths similar to their own research theme, or those that could be considered in their research theme but have not yet been actually considered. Examples of this function are shown in Fig. 13. First, the user finds an upward scenario related to an item of his or her original idea. Then, he or she follows a downward scenario.

Figure 14 shows a recommended scenario path leading to the ethics level. Here, the ethical scenario relates to the idea of paragraph vectors (Le and Mikolov 2014). The process occurs in two elements: the acquisition of path (A) by querying an item and the acquisition of path (B) by querying item (e) from the first result. This scenario path recommendation is made by calculating the similarities between the query path and each candidate path accumulated in the knowledge base. The scenario path recommendation of Dfrome's editor is described in Sekiguchi and Hori (2018a). 
Table 2 Summary of data used in the user studies [group A is listed in Sekiguchi and Hori (2019) and refers to (IEEE 2016, 2017; AI Network Society 2017a, b, c; Future of Life Institute 2017; Oka and Morimoto 2015, 2016; Nickel et al. 2016; Le and Mikolov 2014; Schmid 2010; Krizhevsky et al. 2012); group B was added later and refers to (AIP 2019; Department of Aeronautics and Astronautics 2019)]

\begin{tabular}{llll}
\hline Group & Document & Details & $N$ \\
\hline A & Ethically aligned design & Principles of version 1 & 4 \\
& Ethically aligned design & Principles of version 2 & 5 \\
& Asilomar AI principles & Ethics and Values & 14 \\
& AI R\&D guidelines & Principles & 10 \\
AI R\&D guidelines & Cases & 10 \\
Drone studies & Cases & 6 \\
Machine learning papers & Overview of the algorithm & 5 \\
& Others & Authors' design etc. & 15 \\
& Website of RIKEN AIP & Introduction of each team etc. & 53 \\
& Website of Department of Aeronautics and & Introduction of each laboratory etc. & 11
\end{tabular}

133

\subsection{Browsing ideas on Dfrome's browser}

To browse ideas in Dfrome's browser, a simplified version of the scenario path recommendation is implemented, as shown in Fig. 15. A query can be formed as keywords or sentences, such as an abstract of a paper. The recommended scenario is a textual description based on the rules of ethical design theory, visually presented as shown in Fig. 14. The details are described in Sekiguchi and Hori (2018b).

\subsection{Knowledge base of Dfrome}

All users having a Dfrome account can save their edited treelike data in the knowledge base in a cloud environment. Each datum can be set to active or removed, and private or public. All active data can be managed in the user's folder, and all active and public data can be searched by any user logged into Dfrome. Once retrieved, these data can then be used for semi-automatic generation and scenario path recommendation. During the first user studies, there were 69 tree-like descriptions in the knowledge base (group A in Table 2) (Sekiguchi and Hori 2019). During the second and third user studies, 64 tree-like descriptions were added to the knowledge base (group B in Table 2). The additional data were then created by referring to the introductory texts of each team of the RIKEN Center for Advanced Intelligence Project (AIP) (AIP 2019), and each laboratory, organization, etc. at the Department of Aeronautics and Astronautics, the University of Tokyo (Department of Aeronautics and Astronautics 2019). ${ }^{4}$

\footnotetext{
4 These materials were chosen as preparatory texts for the user studies to create an environment in which the experimental subjects could interact with data related to their own research themes.
}

\subsection{Calculation of editorial support by semi-automatic generation}

Figure 16 illustrates the basic process of semi-automatically mapping items. First, Dfrome parses the input text from which the items are formed. To input the text, the user opens a window from the context menu, inputs the text, and clicks the execute button. Dfrome then parses the text into sentences and their syntax. Sentences having certain verbs, such as "apply," "develop," and "realize," are transformed into passive voice. Dfrome constructs two types of items for each sentence: items for personal reasons (see Fig. 5) and those that describe changes in the hierarchical representation of artifacts or effects on me.

Second, Dfrome positions the generated items along the causality directions: vertically in the hierarchical representation of artifacts and horizontally in the space of personal reasons and effects on me. Dfrome contains many active and public tree-like data in its knowledge base, and it provides representation fields. Each mesh, divided at a constant pitch in the direction of the causal relationship, contains several items. Dfrome calculates the similarities between a candidate item and the items in each mesh, orders the similarity values, and calculates a score by averaging the top $A \%$ of the similarities in each mesh (e.g., $A=$ 10). Dfrome then selects the mesh having the best (minimum) score as the placement of the candidate item.

Third, Dfrome orders the items into the directions of their comprehensiveness. Each item is placed at its detected mesh position as described above. When more than two items occupy one mesh, these items are arranged at equal pitches in directions (white arrows) orthogonal to the causality direction.

Items are connected by arrows as depicted in Fig. 17. To add data to the representation field, users open a window from the context menu and click the execute button. Dfrome then retrieves the representation field data. Next, Dfrome considers all potential arrows among the items of 
the representation field. Cases that can be logically drawn by ethical design theory satisfy any of the following criteria:

1. Both root and target items are in the personal-concerns space $(\mathrm{P})$, and the target item lies to the right of the root item.

2. The root item is in $\mathrm{P}$, and the target item is in the hierarchical representation of artifacts $(\mathrm{H})$.

3. Both items are in $\mathrm{H}$, and the target item lies above the root item.

4. The root item is in $\mathrm{H}$, and the target item is in the effectson-me space $(\mathrm{E})$.

5. Both items are in $\mathrm{E}$, and the target item lies to the right of the root item.

Next, Dfrome calculates whether a similar arrow exists among its knowledge base. Dfrome calculates the similarity $_{\text {total }}$ between each potential arrow and the active and public arrows in the knowledge base. If any similarity is below a certain threshold, Dfrome draws a new arrow.

\section{Hypotheses}

Now that we have reviewed the theoretical background of ethics and have provided an overview of Dfrome, we can proceed to user studies. We first organize our hypotheses to be verified with the user studies.

First of all, we define the criteria of creative products as originality and practicality. Ethics can be considered to support such creativity work and this effect is what this paper is to verify. Therefore, the main hypothesis is as follows:

\section{Hypothesis 1 Ethics support creativity (i.e., creativity sup- port effect of ethics exists).}

Here, practicality and originality were also the criteria introduced by Finke et al. (1992), and these reconsiderations corresponded to specific and generic critiquing mechanism (Fischer et al. 1993), as mentioned in Sect. 2.

The mechanism of the creativity support effect of ethics can be explained by the ethical design theory. A visually modeled explanation of the mechanism by the theory is as shown in Fig. 18. This image presents how skepticism at a higher level widens the conceptual space at the lower levels. As is shown in Fig. 18, we can first assume that one problem has several solutions. Then, higher objectives, such as solving the problem, are not doubted in the design, because they become the preconditions from which the designer realizes lower-level design. Then, the designer reconsiders the higher objectives of his or her work from the ethics level in the
Table 3 Two elements of creativity support effect of ethics

\begin{tabular}{lc}
\hline Direction in hierarchy & Operation in design \\
\hline Vertical & A designer doubts their own ideas \\
& from the higher levels including \\
the ethics level as depicted (A) in & Fig. 18 \\
Horizontal & A designer becomes aware of a \\
& wider conceptual space of design \\
& solutions as depicted (B) in \\
& Fig. 18 \\
\hline
\end{tabular}

vertical direction. Finally, he or she consequently becomes aware of a wider conceptual space of design solutions, as depicted as (B) in the horizontal direction.

In relation to the plurality of design solutions shown in Fig. 18, we can refer to the discussion by Whitbeck (1998) as mentioned in Sect. 2. Additionally, in relation to the point of how propositions at the higher levels become preconditions, we can understand them as Wittgenstein's hinges (Wittgenstein 1969) as discussed in (Sekiguchi et al. 2010; Sekiguchi and Hori 2018a, 2019) and in Sect. 3 of this paper.

Then, we can derive the two elements of the creativity support effect of ethics vertically and horizontally, as is shown in Table 3. The vertical element shows that a designer comes to doubt his or her work at the higher levels, including the ethics level. The horizontal element shows that the designer becomes aware of a wider conceptual space of design solutions.

We can next compare the two elements in Table 3 with the two criteria for creative products, originality and practicality. The vertical element corresponds to the practicality for realizing social values. When items in a tree-like description are seamlessly connected from the lower levels to the ethics level using arrows, the scenario becomes practical for realizing ethical objectives. The horizontal element corresponds to originality. When more items are distributes at the lower levels, it means that the designer has improved his or her originality, because design solutions are considered more comprehensively (e.g., a designer can become aware of alternative solutions from other disciplines, can merge these ideas with his or her own technologies, etc.). Thus, we can set a sub-hypothesis as follows:

Hypothesis 1.1 Creativity support effect of ethics comprises the vertical and the horizontal elements (i.e., creativity support effect of ethics can be explained by the ethical design theory). 
Then, Hypothesis 1.1 has two sub-hypotheses, too. Firstly, we can hypothesize that the effect to enhance the originality becomes greatest when design ideas are doubted from their ethics. Of course, it is true that the creativity support effect can be realized without introducing ethics. However, as suggested in Fig. 18, the higher the design solutions branch, the more comprehensive the design solutions become at the lower levels. Therefore, because ethics is at the highest level, it is reasonable to consider that the ethics level realizes the widest conceptual space. Thus, we can consider that a designer can consider the widest range of design solutions when he or she reconsiders design ideas with regards to their most fundamental purposes. Therefore, we can set a subhypothesis as follows:

Hypothesis 1.1.1 Creativity support effect becomes greatest when design ideas are reconsidered from the ethics level.

Secondly, we can predict that the distance between the target level and the ethics level is in proportion to the difficulty in experiencing the creativity support effect of ethics. A designer who initially sets the parts level as the target level in design will probably experience more difficulty doubting from the ethics level. Nonetheless, he or she will obtain a wider conceptual space for design solutions than a designer who initially sets the interaction level as the target level, because the changes of the levels in the hierarchy by the former designer is larger (higher) than the latter one. Therefore, we can set the other sub-hypothesis as follows:

Hypothesis 1.1.2 Distance between the target level and the ethics level is in proportion to difficulty in experiencing the creativity support effect of ethics.

Then, we assume that clearly applying the ethical design theory to design ideas promotes ethical designs. It also enables us to directly observe the creativity support effect. For doing these, we set Dfrome as an experimental environment because Dfrome supports practicing the ethical design theory and thus is the most effective environment to observe such effect. Therefore, we can set the other two hypotheses as follows:

\section{Hypothesis 2 Ethical design theory promotes introducing ethics into design.}

Hypothesis 3 Dfrome supports practicing ethical design theory.

Finally, when the above-mentioned hypotheses are confirmed, we can consequently confirm that Dfrome supports creativity. The last hypothesis is as follows:

\section{Hypothesis 4 Dfrome supports creativity.}

In short, Hypothesis 1 is the focus of the paper. The Hypothesis 2 and 3 reflect the means to realize this verification. The Hypothesis 4 reflects the verification of the other three hypotheses. Although the Hypothesis 4 is one of the reasons for providing Dfrome, we omit its discussion because its conclusion can be drawn from the conclusions of the other hypotheses.

By conducting user studies, we thus verify the existence of the creativity support effect of ethics by evaluating these hypotheses.

\section{Settings of the user studies}

We deal with three kinds of user studies to verify the existence of the creativity support effect of ethics. These include (a) stakeholder impact studies, (b) editing-idea studies by students, and (c) editing-idea studies by professionals. Thus, in our analysis and discussion, we basically compare results by grouping them into a group comprising the evaluating impact study of (a) and another comprising the editing ideas studies of (b) and (c).

We next describe the common settings of the user studies and typical settings of each user in order. The studies also include an exceptional case (e.g., the semi-automatic generation of items not used by a subject and additional explanations provided in the experimental instruction for answering a question form a subject). The influence of these exceptions on the study results are discussed in Sect. 7.

This paper reports results of newly conducted user studies and those already published (Sekiguchi and Hori 2019) to realize a more comprehensive analysis, including an update of interpretations and comparisons between the new and former findings.

\subsection{Common settings}

Our expectation (i.e., ethics supports creativity) was withheld from the subjects. Subjects were instructed on using Dfrome's scenario path recommendation, which allowed them to extend their themes into similar or divergent scenarios. However, users were not informed that we expected their themes to extend to the ethics level or that their design ideas should be reconsidered at lower levels. Especially, the intention of the task (i.e., to acquire novel awareness and reconsideration of original research or business themes) was not disclosed.

Whether our expectations were gleaned by the subjects was assessed in a follow-up questionnaire, followed by a hearing based on a task-paper sheet, questionnaire answers, a record of the operation screen, and other relevant themes. 
Table 4 Overview of the research themes in the stakeholder impact studies

\begin{tabular}{llll}
\hline $\begin{array}{l}\text { Order } \\
\text { paper) }\end{array}$ & $\begin{array}{l}\text { Id (in } \\
\text { this } \\
\text { paper }\end{array}$ & Theme \\
\hline 1 & A & Master in 1st grade & Localization and drone \\
2 & B & Master in 1st grade & Aeronautical data analysis \\
3 & C & Master in 2nd degree & Deep learning \\
4 & D & Master in 1st degree & Drone \\
\hline
\end{tabular}

Data of the state of the subject, the introductory session, the hearing, and related data were recorded as far as possible. The experiments were conducted in Japanese. Although Dfrome is only available in English, the web search and task paper sheet can be conducted in any language.

\subsection{Stakeholder impact study (laboratory experiment $)^{5}$}

In this experiment, subjects were asked to consider the impact of their own research and development on stakeholders when realized. The user studies were conducted 17-25 October 2018.

\subsubsection{Procedure}

The user studies proceeded as follows. Dfrome use and user tasks were explained to subjects (about 5 min each); a subject completed set tasks $(20 \mathrm{~min} \times 2$ frames $=40 \mathrm{~min})$; a subject answered a questionnaire and attended the hearing. The following subsections explain the environment, subjects, tasks, questionnaires, and hearings.

\subsubsection{Environment}

The environment was schematized and photographed in Fig. 19. Each user study was performed by a single participant.

\subsubsection{Subjects}

Four students enrolled in Masters' courses at the AI Lab at the University of Tokyo participated in the experiment. The theme patterns are summarized in Table 4 .

\subsubsection{Task}

Each subject was given an A4-size paper sheet with the following question written at the top.

\footnotetext{
$\overline{5}$ This subsection is a review of Sekiguchi and Hori (2019).
}

What kind of impact will the result of your research and development have on the stakeholders when it is realized? Please fill in the blank freely.

Please speak out loud as you think. An Internet environment is available. The time is 20 minutes for two frames. Please use the tool in only one frame (explanation will follow).

As shown here, there were two frames for each user study: one with Dfrome and the other without. Before explaining the frame using Dfrome, we briefly explained Dfrome. The explanatory text was spread over two pages, but its contents were those shown in Fig. 15. The following two hints were also provided.

Let us input an abstract of your research paper.

If there are insightful descriptions in the recommended scenario, let us input them next.

In the user studies, the use conditions of Dfrome (first or second frame) were rotated in the order of the subjects' participation. Specifically, subjects A and C used Dfrome in the first frame, and B and D used it in the second. Because the subjects' participation order was determined by external factors, such as the subject schedules, the use conditions of Dfrome can be regarded as reasonably randomly allocated.

\subsection{Editing ideas study (laboratory experiment)}

In this experiment, the subjects were required to edit their own ideas in the tree-like format of ethical design theory. These user studies were conducted from 28 February to 7 March 2019.

\subsubsection{Procedure}

The user studies proceeded as follows. First, the instruction paper of the user study was explained to the subjects. Then, the subjects were given a brief tutorial on Dfrome. The subject then completed an assigned task and answered a questionnaire. The subject then signed the participation agreement form and attended a hearing.

\subsubsection{Task}

The assigned task was explained on the instruction paper given to the participant. The contents of the instruction items were as follows:

1. Please use the support tool (Dfrome: https://www.dfrom e.com)

2. Please use semi-automatic generation functions to query the research theme you have prepared today. 
Table 5 Overview of research themes in editing ideas study

\begin{tabular}{|c|c|c|c|}
\hline Order & Id & Grade & Theme \\
\hline 1 & $\mathrm{E}$ & Bachelor in 4th grade & Anomaly detection \\
\hline 2 & $\mathrm{~F}$ & Bachelor in 4th grade & $\begin{array}{l}\text { Dynamic mode decompo- } \\
\text { sition }\end{array}$ \\
\hline 3 & G & Master in 1st degree & $\begin{array}{l}\text { Unmanned aerial vehicles } \\
\text { (Drone) }\end{array}$ \\
\hline 4 & $\mathrm{H}(=\mathrm{D})$ & Master in 1st degree & $\begin{array}{l}\text { Differential equation } \\
\text { modeling }\end{array}$ \\
\hline 5 & $\mathrm{I}(=\mathrm{B})$ & Master in 1st degree & Text generation \\
\hline
\end{tabular}

3. Please edit the obtained result to clarify the ideas and their positions. The task-execution time is $20 \mathrm{~min}$. Please use the scenario path recommendation, if necessary.

4. Please answer the questionnaire distributed separately after completing the task.

Additional instructions were given as follows:

5. Please use English in the support tool.

6. Please answer the questionnaire in either English or Japanese.

7. An Internet environment (Google etc.) is available.

8. Please contact the proctor if there are any problems such as poor physical condition, tools not working, or any other emergency situation.

\subsubsection{Subjects}

Five students participated in each user study. A brief overview of the research themes of the subjects are as shown in Table 5. Two of them also overlapped in Table 4.

\subsubsection{Environment}

During the instruction session and during the task, Dfrome ran on the laptop of the proctor (one of the authors).

\subsubsection{Explanation of Dfrome}

Before completing the task, students were given an explanation of Dfrome. This brief tutorial comprised two parts: how to view and how to use. The first part was concerned with ethical design theory, because it defined the perspectives and explained how to write and read the tree-like descriptions. However, because the perspectives of ethical design theory were too complicated for an immediate understanding, we simplified them to the descriptions shown in Fig. 20. The grammar is also described in this part (see Fig. 5). The second part concerned the use of Dfrome. The explanation contents were nearly identical to the relevant parts of the functions used in the user studies described on the explanation page of Dfrome. ${ }^{6}$ The semi-automatic generation functions and scenario path recommendation were also demonstrated in this part.

\subsection{Editing ideas study (field experiment)}

To evaluate the actual use of Dfrome, field experiments were conducted from 8 April to 3 July 2019. The settings were nearly identical to those described in Sect. 6.3. However, they differed from the laboratory settings in three ways. First, there was a longer experimental period ( 2 business days across approximately 1 week). The actual experimental period varied from 5 days to nearly 2 months, depending on the subjects' schedules. Within this longer period, the subjects were expected to use the scenario path recommendation as well as its basic editing functions. The second difference was the location of the task (freely selectable by the subject with his or her own computer). The final difference was the professional level of the subjects (experts rather than students). The procedure, task, and subjects of the field experiments are described below.

\subsubsection{Procedure}

The user study comprised three parts. On the instruction day, an instruction paper was explained to each subject, and each signed the experimental agreement form. Next, the subject received a brief tutorial and demonstration of Dfrome. The subject then conducted a task alone. On the hearing day, each study author returned a questionnaire, conducted a hearing with the subject, and concluded the study.

\subsubsection{Task}

The task to be completed was explained on the instruction paper. The contents of the instruction items are as follows:

1. Please use the support tool (Dfrome: https://www.dfrom e.com)

2. Please use the semi-automatic generation functions to query your own theme. The functions contain the generation of items and connection of them with arrows.

3. Please edit the obtained result to clarify the ideas and their positions.

4. Please use the scenario path recommendation, if necessary.

5. Please complete the task over 2 days (or 2 business days in your spare time. The approximate deadline is 1 week after signing the agreement form).

$\overline{6}$ https://www.dfrome.com/how_to_editor. 
Table 6 Overview of the subjects in the editing ideas study

\begin{tabular}{|c|c|c|c|c|}
\hline Order & Id & Education & Occupation & Theme \\
\hline 1 & $\mathrm{~J}$ & $\mathrm{PhD}$ & $\begin{array}{l}\text { Software } \\
\text { engineer in a } \\
\text { startup }\end{array}$ & IoT for aquaculture \\
\hline 2 & $\mathrm{~K}$ & $\mathrm{PhD}$ & Researcher & $\begin{array}{l}\text { (1) Health monitoring } \\
\text { for satellite, (2) Topic } \\
\text { model }\end{array}$ \\
\hline 3 & $\mathrm{~L}$ & M.Eng & Entrepreneur & Drone business \\
\hline 4 & M & $\mathrm{PhD}$ & Researcher & Disaster damage mapping \\
\hline
\end{tabular}

Table 7 Classification of the subjects' experiences

\begin{tabular}{|c|c|c|c|c|}
\hline & & $\begin{array}{l}\text { Did the subjec } \\
\text { higher levels? }\end{array}$ & loubt from the & \\
\hline & & $\begin{array}{l}\text { Yes (includ- } \\
\text { ing the ethics } \\
\text { level) }\end{array}$ & $\begin{array}{l}\text { Yes (without } \\
\text { the ethics } \\
\text { level) }\end{array}$ & No \\
\hline Did the subject & Yes & $\mathrm{i}$ & ii & iii \\
\hline $\begin{array}{l}\text { become aware of } \\
\text { a wider conceptual } \\
\text { space? }\end{array}$ & No & iv & $\mathrm{v}$ & vi \\
\hline
\end{tabular}

6. Please answer the questionnaire distributed separately after completing the task. I will collect it later.

7. Please cooperate with the interviewer when the questionnaire is collected.

Additional instructions were given as follows:

8. Please use English in the support tool.

9. Please answer the questionnaire in either English or Japanese.

10. Please contact the study author if there are any problems such as poor physical condition, tools not working, or any other emergency situation.

\subsubsection{Subjects}

The subjects are overviewed in Table 6 .

\section{Results of user studies}

To appropriately report the results of the user studies, we applied a categorization. Table 7 classifies subjects' experiences by the two elements discussed in Sect. 5. As shown in this table, we set six $(=3 \times 2)$ patterns: $i$ to vi. Concerning the first element (i.e., a designer doubts his or her own ideas from higher levels, including the ethics level), we set a question "Did the subject experience doubt at the higher levels?" and set three answers. Regarding the experiences of subjects whose answer is considered to be was "Yes," there were two possible answers: including the ethics level and without the ethics level. Thus, we divided these as two answers into "Yes (including the ethics level)" and "Yes (without the ethics level)." The third captured the situation in which the answer of the question is considered to be "No." Concerning the second element (i.e., a designer becomes aware of a wider conceptual space of design solutions), we set a question "Did the subject become aware of a wider conceptual space?" and set two answer options: "Yes" and "No." The subjects' experiences were also gleaned from the user-action logs, operation screen records, questionnaire responses, discussions during the hearing, and other relevant records. The results also revealed limitations of the present version of ethical design theory. These results are separately discussed in later subsections.

\subsection{Overview of the results}

The results were classified by the patterns shown in Table 7 . Table 8 presents an overview of the results of the stakeholder impact study (laboratory experiment), and Table 9 presents an overview of results of the editing-ideas studies (laboratory and field experiment). In Table 9, there were ten themes by nine subjects because subject $\mathrm{K}$ dealt with two themes, (1) and (2). To better understand the probability of the occurrence of the creativity support effect of ethics, we calculated the rate of the number of corresponding results for each pattern. If there was more than one experiences in a pattern of a subject's work, we counted one. If we evaluated that there was no correspondence, we added "None" and counted zero. The boldface experiences having asterisks in the tables are described in detail in Sect. 7.2.

The most important point at this stage was whether we could observe pattern $i$, which corresponds to a confirmation of the creativity support effect of ethics, including doubting from the ethics level and becoming aware of a wider conceptual spaces. In Table 8 , half of the subjects ( 2 of 4 ) succeeded in realizing pattern i. In detail, there were four total experiences: three experiences were realized by subject A and one by subject D; three were with Dfrome and one was with Google instead. Then, all four subjects doubted their own ideas at the interaction level or below (pattern ii or v). Furthermore, two of them generated a new application (pattern ii). There were no correspondences in patterns iii and iv, and, because pattern vi is the default situation of the task in which the two elements of the expected creativity support effects by ethics cannot be observed, we omitted those details.

As seen in Table 9, only one subject experienced the creativity support effect of ethics (pattern i). That without ethical thinking was not observed (pattern ii). However, Dfrome succeeded in helping 6 out of 9 subjects recognize the existence of the ethics level (pattern iv). Thus, three of nine succeeded using the semi-automatic generation function (i.e., 


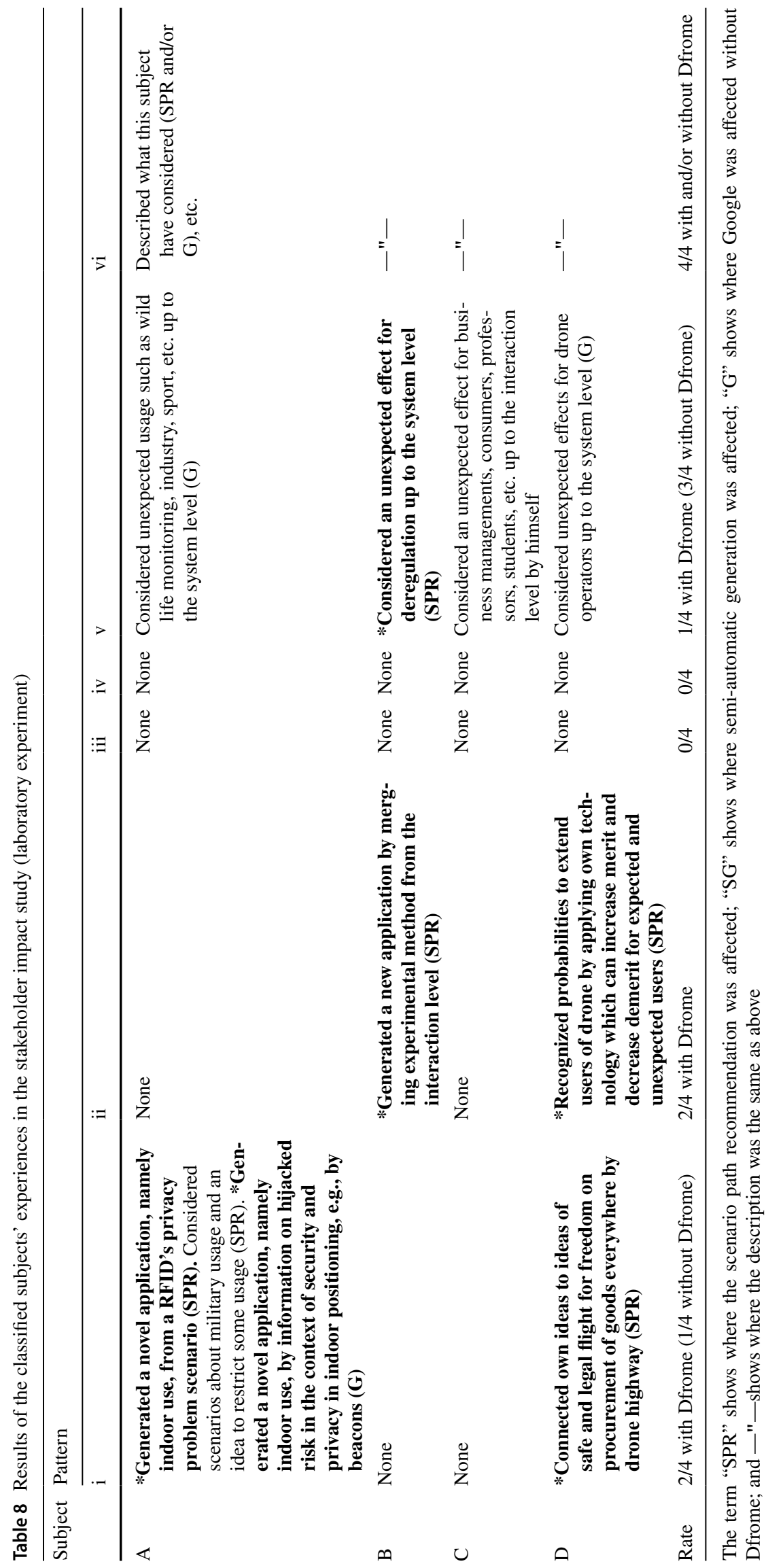




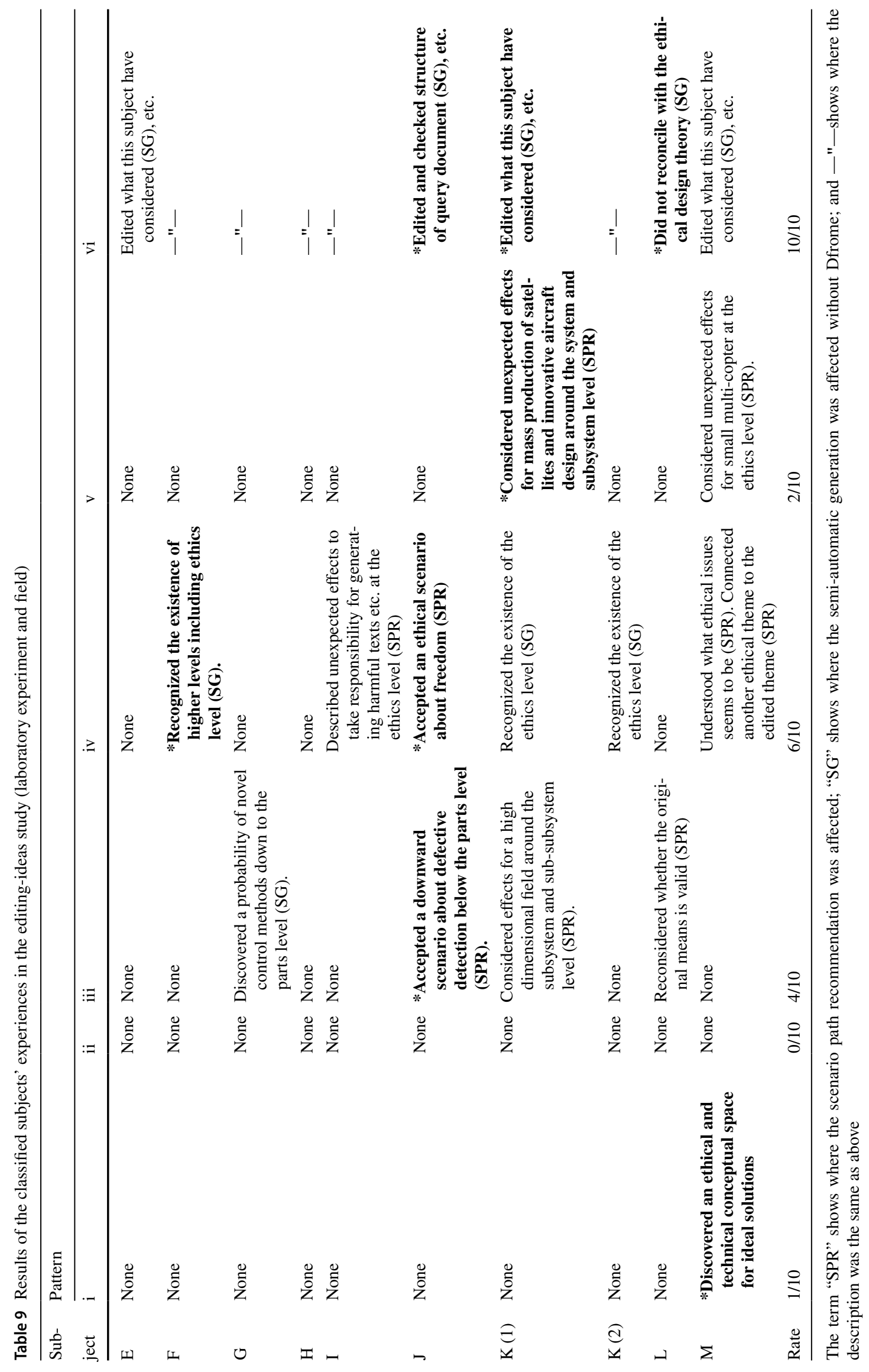


visually recognized the level in the perspectives defined by the ethics level). Then, we could confirm that there were extensions of the tree-like descriptions of their own ideas in one direction [i.e., downward (pattern iii) or upward (pattern v)]. Regarding pattern vi of editing ideas studies, we describe remarkable results in Sect. 7.2.6.

We then overviewed the results concerning the orthogonal representation of personal concerns. Regarding this point, we looked into the results of the user studies for idea editing, because the orthogonal representation was explicitly handled in the user studies, because they presented the representation field of the Dfrome's editor, whereas this dimension implicitly dealt with in Dfrome's browser, which was used for stakeholder impact study. In the editing task, all subjects successfully edited their own tree-like descriptions in the space for personal reasons. We could observe that some subjects added items to the space for personal reasons by themselves. And there were a typical operation to transfer items positioned within the hierarchical representation of artifacts using Dfrome's semi-automatic generation function into the space reserved for personal reasons. Regarding the personal reasons representation, among the nine subjects, one subject performed both of the operation (subject F), other four subjects added items (subject $\mathrm{E}, \mathrm{J}, \mathrm{L}$ and $\mathrm{M}$ ), and the other four subjects transferred items (subjects G, H, I and K).

However, the definition of describing the effects on me tended to confuse the subjects. In fact, subject $G$ initially understood to use the space for describing effects on me (the designer) as with the higher levels in the hierarchical representation of artifacts. subjects $\mathrm{K}$ and $\mathrm{M}$ explicitly commented on this confusion in the hearings.

\subsection{Details of the remarkable results for each pattern}

Then, we describe the details of remarkable results in Tables 8 and 9 with bold fonts and an asterisk mark. We first describe the results of pattern $i$, which will verify whether ethics can support creativity. We then describe the results of patterns ii-vi. Here, the subjects' comments (in double quotations) were translated by the authors from Japanese into English. The comments in parentheses are supplementary explanation by authors.

\subsubsection{Pattern i: the subject doubted his or her ideas from the ethics level and became aware of a wider conceptual space}

Subjects' experiences fitting this pattern were identifiable from the results of the stakeholder impact study and the editing-ideas studies. For example, after using the Dfrome browser, subject A replied in the questionnaire that "although I had thought about the benefits up to now,
I had not thought about the risks, so I gained awareness of the risks" and "I have been paying attention to the outdoor use (of drones), but since I felt they would likely be used indoors, I would like to think about their indoor use and problems." Subject A's ethical concern was triggered by the collection of radio frequency identification (RFID) data in shopping malls, which impacts customer privacy. After using Dfrome, this subject considered a novel application of indoor use and its ethical problems as well.

Subject D attempted to connect his original idea of a drone to the novel viewpoint obtained from the task execution, such as "safe and legal delivery drone flights along drone highways leads to freedom of procurement of goods everywhere." Notice that when this subject asked the study author about the granularity of an utterance concerning "think aloud," the study author answered that the subject should "please talk aloud e.g., when awareness occurs." However, it is still significant that this subject spontaneously realized this connection to the scenario that was a part of an ethically rich tree-like description that had been registered based on discussion with an $\mathrm{AI}$ ethics/AI expert and public law expert.

Pattern i can be realized without Dfrome and without directly considering the ethical design theory. One example is that subject A generated a new application by accepting a hijack-risk scenario connected with ethical terms (i.e., privacy and security). This subject watched a website Wurms (2016) found in a Google search and realized that beacon infrastructure has the potential to be hijacked. Thus, he generated a new drone application to replace the infrastructure in an environment where global positioning system (GPS) could not be used by hijackers to commit harmful actions.

As an example of widening the conceptual space after using Dfrome's editor, professional-subject $M$ stated in the hearing "On writing (the ideas), the items at the top can be collected into one theme that I would like to follow; however, (I consider that) a few more items at the top should be added" and described to the questionaire that "(I) would like to increase the number of items at the ethics level or the interaction level, (considering the) impacts on society." In the hearing, subject $\mathrm{M}$ further commented that writing research papers seemed to be easier at the lower levels, but that it became difficult at higher levels. Subject M added that "some kind of theme would be ideal if the theme can be written as a paper and has a great social impact; and reconsideration seems to be demanded because such a theme is not easily conducted." Figures 21 and 22 show the process by which subject $\mathrm{M}$ edited his ideas. Clearly, this subject attempted to extend his expression toward the higher-level theme. Note that this subject did not use semi-automatic mapping. Nonetheless, the significance of showing the validity of the creative effect by ethics is the same. See Figs. 21 and 22. (Hereafter, we added spouts to improve readability of characteristic items. When the document file is in PDF format, all the items are to be readable by being zoomed in.) 


\subsubsection{Pattern ii: the subject doubted his or her ideas from the interaction level or below and became aware of a wider conceptual space}

This pattern was typically observed when a subject obtained information that contained both technologies and unexpected effects without ethical perspectives and/or terms. This pattern was observed only in the stakeholder impact study in the Dfrome condition. For example, subject B, who dealt with data analysis for aircrafts, generated an idea that, "if the results of the data analysis can be checked against experimental data, then aerodynamic engineers will change to work harder to improve the aerodynamic model." Here, this subject seemed to be affected by the scenario: "if his work will be realized, then students will change to improve motivation for learning," whose tree-like description was registered by one of the study authors by referring to (AI Network Society 2017c). The subject's input text for this recommendation was "By considering uncertainty of flight data of aircrafts, we could estimate aerodynamic coefficients more accurately." Because the motivation of improvement by the engineers is expressed at the interaction level, he generated a novel design solution below the ethics level (pattern ii).

Another example is that subject $\mathrm{D}$ recognized that probabilities to extended users of drone technologies by applying his own technology, which could increase merit and decrease demerit for expected and unexpected users. This case was observed when this subject viewed a recommended scenario of a multi-drone system that increased the reliability of sensed data and whose tree-like description was registered by one of the study authors by referring to (Yanmaz et al. 2017).

\subsubsection{Pattern iii: the subject became aware of a wider conceptual space, but did not doubt his or her ideas from the higher levels}

This pattern was typically observed in a lower-level scenario considered by subject $\mathbf{J}$ [see the region labeled (b) in the second panel from the top in Fig. 23].

\subsubsection{Pattern iv: the subject doubted his or her ideas from the ethics level, but did not recognize a wider conceptual space}

This pattern was observed in subject $\mathrm{J}$ whose process is Figs. 23 and 24, who presented an upward scenario recommended by Dfrome [see (a) in Fig. 23]. Although this subject tested the scenario path recommendation in the upward direction, the scenarios were not connected to some novel path at the lower levels. Therefore, the higher-level reconsideration did not translate into a lower-level reconsideration.
The other ethical scenarios considered by subject $\mathrm{J}$ had already been tackled by this subject's startup project. Thus, he had not related to the expected creativity support effect. The subject commented that "I used (Dfrome) to arrange the document of the mission and work of my company," and "I did not gain big awareness, because I did not use it to obtain a novel idea. If the tool is (meant) for obtaining awareness, it would be better if I had been informed of this beforehand (and the results may have differed)."

In another example of pattern iv, subject $F$ replied in the questionnaire that "I became aware that in my research, at least in my abstract, there was not consideration at higher levels like ethics and interaction levels and the description focused on the lower parts." This subject also answered that "it might be good to further consider the missing part of my research." However, this subject did not apparently reconsider any novel design solutions from the higher levels. The editorial process of Subject $\mathrm{F}$ is outlined in Fig. 25. This process confirmed that subject $\mathrm{F}$ attempted higher-level editing.

\subsubsection{Pattern v: the subject doubted his or her ideas from the interaction level or below, but did not become aware of a wider conceptual space}

This pattern was typically observed when the subject was recommended an unexpected effect's scenarios without ethical perspectives, but he she did not seem to have the intention to change his or her technology. For example, subject B considered an unexpected effect of his technology for deregulation up to the system level when he was recommended a scenario about drones flying along drone highways, which aimed to revise the safety rules concerning the Civil Aeronautics Act and other general laws, as was registered by one of the authors by referring to (Terada 2018) during collaboration (Sekiguchi et al. 2018). He recognized the probability to contribute to some deregulation by improving accuracy of estimation. However, he did not intend to consider alternative solutions for these issues. One of the probable reason is that his experimental condition started without Dfrome and had already developed, such that he lost his intention to doubt his original ideas in the latter frame of Dfrome application.

Another example is that subject $\mathrm{K}$ considered unexpected effects for mass production of satellites and innovative aircraft designs around the system and subsystem level when he tried using the scenario path recommendation function of Dfrome's editor during a hearing. In this case, he did not have any intention to change his original ideas, because he felt that he did not have enough time to stop and think while talking. 
Table 10 Results and details of pattern i by successful subjects A and D using Dfrome

\begin{tabular}{|c|c|c|c|}
\hline No. & Hypothesis & Result & Details \\
\hline 3 & Dfrome supports practicing ethical design theory & $\mathrm{P}$ & $\begin{array}{l}\text { Dfrome provided scenario paths by scenario path recommendation function } \\
\text { whose grammar is defined by the ethical design theory. And some of the } \\
\text { scenarios were accepted by the subjects when the successful cases were } \\
\text { experienced }\end{array}$ \\
\hline 2 & $\begin{array}{l}\text { Ethical design theory promotes introducing eth- } \\
\text { ics into design }\end{array}$ & $\mathrm{P}$ & $\begin{array}{l}\text { Both of the successful subjects introduced ethical terms connected to techni- } \\
\text { cal context by considering the scenario paths defined by the ethical design } \\
\text { theory, i.e., (1) privacy problem by RFID, (2) military usage whose original } \\
\text { scenario was connected to drone and peace issues and (3) safe and legal flight } \\
\text { for freedom on procurement of goods everywhere by drone highway }\end{array}$ \\
\hline 1 & Ethics support creativity & $\mathrm{P}$ & $\begin{array}{l}\text { Both of the successful subjects became aware of a wider conceptual space and } \\
\text { generated a novel design solution by considering the above-mentioned ethical } \\
\text { terms, i.e., (1) using drone indoors, (2) restricting some usage and (3) apply- } \\
\text { ing his drone technology to drone highway }\end{array}$ \\
\hline
\end{tabular}

$P$ Positive, $N$ Negative

Table 11 Results and details of pattern i by successful subject A without Dfrome

\begin{tabular}{llll}
\hline No. & Hypothesis & Result & Details \\
\hline 3 & $\begin{array}{l}\text { Dfrome supports practicing ethical design theory } \\
\text { Ethical design theory promotes introducing ethics into } \\
\text { design }\end{array}$ & $\mathrm{N}$ & $\begin{array}{l}\text { Dfrome was not used when this case was experienced } \\
\text { He must not be clearly conscious of the ethical } \\
\text { design theory at that time because subject A was } \\
\text { not familiar to the ethical design theory and did not } \\
\text { use Dfrome }\end{array}$ \\
1 & Ethics support creativity & $\mathrm{P}$ & $\begin{array}{c}\text { Subject A introduced ethical thinking and widened } \\
\text { his conceptual space by considering information of } \\
\text { hijacking risk in the context of security and privacy } \\
\text { in indoor positioning, e.g., by beacons }\end{array}$ \\
\hline
\end{tabular}

$P$ positive, $N$ negative

\subsubsection{Pattern vi: the subject neither doubted his or her ideas from the ethics level, nor became aware of a wider conceptual space}

This pattern was typically observed when a subject simply edited his or her own ideas using the Dfrome editor. However, such cases validated the ethical design theory to some extent. For example, subject $\mathrm{K}$ answered in the questionnaire that "unexpectedly, research topics can be described in hierarchical form, such as subsystem-system-interaction-ethics." Figs. 26 and 27 clarify the tree-like description of the editing process of one of the two editorial results of subject $\mathrm{K}$.

Subject $\mathrm{J}$ acquired creativity support not only by describing ideas in Dfrome, but also from another aspect of the semiautomatic generation. In the questionnaire, this subject mentioned that the semi-automatic generation probably obtained some inappropriate points. He then mentioned that this inappropriateness (i.e., failure to express causality) seemed to be caused by a deficiency in the query document extracted from the webpage of the startup company at which he works, which was marred by logical leaps and missing words. These points were acknowledged by the company but not clearly described on this part. Therefore, subject J could check the structure of the query document during the user study.

Other typical examples of pattern vi were found when subjects ignored a scenario recommended by Dfrome.

\subsection{Extracting key factors to succeed and un-succeed in experiencing pattern $i$}

To extract key factors of success in experiencing Pattern i, we quantitatively investigated the results. This analysis provided knowledge utilized for more intentional realization of the creativity support effect of ethics. 
Table 12 Results and details of pattern i by successful subject M with Dfrome

\begin{tabular}{|c|c|c|c|}
\hline No. & Hypothesis & Result & Details \\
\hline 3 & Dfrome supports practicing ethical design theory & $\mathrm{P}$ & $\begin{array}{l}\text { Dfrome provided the visual image of the perspectives defined by the ethical } \\
\text { design theory }\end{array}$ \\
\hline 2 & $\begin{array}{l}\text { Ethical design theory promotes introducing eth- } \\
\text { ics into design }\end{array}$ & $\mathrm{P}$ & $\begin{array}{l}\text { The visualized image of the perspectives and the tree-like description of his } \\
\text { own ideas make subject D understand that there exist the level corresponds to } \\
\text { social impacts (ethics level) }\end{array}$ \\
\hline 1 & Ethics support creativity & $\mathrm{P}$ & $\begin{array}{l}\text { The subject became aware of a probability of a design solution that satisfy both } \\
\text { values at the higher levels including the ethics level and those at the lower } \\
\text { levels like the parts level }\end{array}$ \\
\hline
\end{tabular}

$P$ positive, $N$ negative

\subsubsection{Key factors in stakeholder impact study}

We first analyzed the results of the stakeholder impact study. Here, we derived three kinds of key factors. The first accounts for the method of providing information (i.e., information should be delivered in a form by which a subject can infer the relations between his or her own theme and the one provided by ethical terms). Here, we evaluated the pattern i results from the hypotheses introduced in Sect. 5 to confirm this key factor. Pattern i with Dfrome is as shown in Table 10 and that without Dfrome (with Google) is shown in Table 11. To correspond to the steps of the user study logics, we reverse the order of the hypotheses. As these tables show, these subjects obtained information on not only ethical issues, but also technologies. This method of providing information was confirmed to allow subjects to easily infer relationships. For example, when subject A was recommended scenarios about privacy problems and the indoor use of RFID technologies, he could infer the relation between his own technology by replacing RFID with his technology. In the other cases, accepted scenarios by subjects related to drones, which was familiar to the subjects. Therefore, these subjects were considered to be able to experience the creativity support effect of ethics.

From Table 11, we can also confirm that neither Dfrome nor the ethical design theory was necessary for experiencing pattern i. It was confirmed that other technologies, such as Google, was able to provide information in the abovementioned way. However, although Google covers various and abundant information, it basically provides a function to allow users to precisely obtain information on what they already want to know. Thus, Google does not tend to change a user's viewpoint. Experiencing the creativity support effect by Google is considered to occur by chance. In fact, the number of successful cases in Dfrome condition was three times that of Google alone. The ethical design theory can also be used to explain the mechanism of the effect and predict the occurrence of it. Similarly, Dfrome can be used to support practical ethical design theory. In other words, Dfrome is guaranteed to provide scenarios that are seamlessly connected from technical to ethical ideas. Additionally, in patterns ii and iii, which correspond to the creativity support effect without ethics, Dfrome had two experiences, whereas Google did not have any. Although subjects were not randomly selected and the number of cases was too small to derive any statistical conclusion, these results support our assertions that clearly applying the ethical design theory and being supported by Dfrome is useful to experiencing the creativity support effect of ethics by guaranteeing this key factor.

Regarding the unsuccessful patterns ii and $\mathrm{v}$ cases, the reason they did not reach pattern $i$ is that the subjects obtained information in a way in which he or she could not connect any ethical terms to their own theme.

The second key factor includes the topics of the subject. There were common conditions among the successful subjects A and D: both of them deal with "drones." In Dfrome's knowledge base at that time, drone contents were abundantly registered (6 of 69) in relation to collaboration (Sekiguchi et al. 2018). As a result, the available paths for scenario path recommendation were abundant, and the corpus for calculating distances between an input text and candidate paths was rich. Thus, we can understand that the quality of recommendation is effective enough because of their topic selection.

The third factor included the personal concerns of the subject. Apparently, subject A was a natural fit for this task. In fact, he generated three of four successful pattern $i$ cases. The task setting itself (i.e., considering the impacts of one's own theme on stakeholders) was considered to enhance the nature of his own theme at the higher levels (i.e., it directed the intention of subject against the outer environment of his own technology). These points relate to a subject's positioning in the orthogonal representation of personal concerns. In other words, it was essential to properly direct a designer's intention in the orthogonal representation of personal concerns. This point was confirmed in the editing ideas studies, as well. 


\subsubsection{Key factors in editing ideas studies}

We next analyzed the results of the editing-ideas studies. Here, we derive two kinds of key factors. The first is the visualized image of the perspectives defined by the ethical design theory (i.e., design from the ethics level). The result of evaluating the pattern i case by the hypotheses introduced in Sect. 5 is as shown in Table 12. As the third aspect of this table shows, the subject became aware of a probability of a design solution that satisfied values at both higher and lower levels. Thus, this subject realized the creativity support effect of ethics spatially without considering concrete ideas. The spatial understanding induced by the visualization of the perspectives was considered essential. Here, the semiautomatic generation did not affect things, because this subject basically did not use the semi-automatic item-mapping function for the task.

The second key factor is the personal concerns of the subject: the same as the third key factor of the stakeholder impact study. Subject M, who realized Pattern i to be an editing ideas study, already had an opportunity to explain his project. He said in the hearing that "Recently, I joined an assessment of the projects of my organization and had to do the similar thing (what I did in this task). I had to explain (my project) by thinking of a story how our research can be useful and what is the challenges and technical solutions. And for the explanation, I did not organize my ideas by beginning the story from technical points but from the higher (levels') points because I thought beginning from the technical points may confuse the listeners but beginning form the higher (levels') points can be more acceptable by listeners."

The other subjects did not experience such reconsideration, even if they understood the same perspectives. When subjects were instructed to edit their own ideas on Dfrome's editor, they actually expanded the current description of their ideas to make them more comprehensive. However, they did not tend to doubt both purpose and means at the same time. For example, subjects sometimes considered extra downward scenario paths without doubting their purposes (pattern iii) (i.e., to make the lower part of the description more comprehensive). Other times, subjects doubted the higher levels without becoming aware of a wider conceptual space for design solution (pattern iv or v) (i.e., to make the upper part of the description more comprehensive). In fact, subject J mentioned that "I did not gain big awareness, because I did not use it to obtain a novel idea. If the tool is (meant) for obtaining awareness, it would be better if I had been informed of this beforehand (and the results may have differed)." In other words, the luck of the intention to reconsider own theme in whole is the key factor for the lack of pattern i success.

\subsubsection{Comparative analysis of the two types of user studies}

Finally, we compare the two types of user studies to obtain further understanding. The pattern i cases from the stakeholder impact study were more semantic. They were triggered by concrete scenarios. On the other hand, the pattern $\mathrm{i}$ case in the editing ideas studies were more spatial, as it appeared in the perspectives of the ethical design theory visualized on Dfrome. As mentioned, the semantic route can be realized without Dfrome if proper information is provided from elsewhere. However, the spatial route is unique to Dfrome because, as far as we know, no other tool has the perspectives seamlessly connected from the technical to the ethics levels in the hierarchical representation of artifacts.

The common key factor showed that proper positioning in the orthogonal representation was essential to promoting ethical design. In the user studies, this point was confirmed both as the user's intention and the task settings. Here, we can compare the task settings. The task in the stakeholder impact study directed subjects to extend their own technologies. However, the task in the editing ideas studies directed subjects to precisely express their ideas on the representation field and, if any, struggle to make the representation more comprehensive without doubting them as a whole. This is one of the main reasons why the stakeholder impact study succeeded in pattern i more than the editing ideas studies.

\subsection{Limitations of the present version of ethical design theory}

Although the results generally supported ethical design theory, the limitations of the theory were also highlighted. We next introduce these limitations to provide an update of the present version of the ethical design theory in Sect. 8. These limitations are described below.

\subsubsection{When the subject emphasized the temporal nature of ideas}

In some cases, the subject emphasized the temporal nature of his or her ideas. For example, subject $\mathbf{J}$ focused on the recursivity of ideas, mentioning that "the dataset seemed to be, well, recursive." The data gathered by the system in this case [the region labeled (c) in the bottom panel of Fig. 24] moved upward after all. However, it will return to the user who installed the product.

In other cases, subject L emphasized evolutionary features, such as company growth and redefinition of ideas. In the hearing, subject $\mathrm{L}$ mentioned that the personal reasons, the hierarchical representation, and effects-on-me factors were not connected as a linear line, but jump to higher layers (= levels) as time passes. This subject also mentioned that "I work for customer basis, so I have no choice but to 
set multiple visions, although (we) share common views on drones or robotics. At a higher layer, I then consider the company's actions as a whole. Therefore, a growing company generates a push-up pressure, and the company thinking changes little by little." The editing process of subject $\mathrm{L}$ (see Figs. 28, 29) showed little of the tree-like features expected in Dfrome editing. Thus, he was not reconciled with the description of ethical design theory.

\subsubsection{When the social context of the subject directed the task execution}

Professionals occasionally allowed their social contexts to direct their task execution. For example, subject $\mathrm{J}$ focused on the mission of the startup at which he works. This subject mentioned the need for editing ideas within the framework of ethical design theory for accounting for the mission. Subject $\mathrm{K}$ highlighted a limitation of collaboration, namely, that "in engineering or similar types of applied research, even when a scenario of a different field can be obtained... the cost (of actually collaborating with researchers in this field) is quite high." Subject L mentioned that as an entrepreneur, he considers different perspectives, as described in Sect. 7.4.1. He was also prohibited from disclosing his company ideas via a confidentiality agreement etc. Subject M mentioned his responsibility of accountability as a unit leader.

\subsubsection{When the subject did not accept the results of the semi-automatic arrow generation}

The results of the semi-automatic arrow generation were not always accepted by the subjects, especially by the experts (see Figs. 21, 22, 23, 24, 26, 28, 29). One apparent reason for this rejection was the untidiness of the idea description. For example, subject $\mathrm{M}$ answered in the hearing that "What I cannot understand was the lack of distinction between the arrow that I originally drew and the arrow that was semiautomatically drawn."

Another apparent reason for rejection was the clarity of the representation field, which already revealed whether or not items were connectable. For example, subject $\mathrm{K}$ answered in the hearing that "the arrows are rather selfevident." Thus, if the representation shows a higher and a lower item, an arrow should then be drawn from the lower to the higher item. Although the arrows are useful guides, the semi-automatic generation can be understood without them.

\subsubsection{When the level definitions are ambiguous}

Some subjects were confused by the level definitions. In fact, the levels were only briefly defined during the instruction time of the user studies, because they were intended to be semi-automatically generated by Dfrome. If the mapping function maps each item at the proper level, the subject can then correctly position his or her ideas. However, subject $\mathbf{J}$ commented that "I could not decide the level of some items, so I vaguely placed those items." Nevertheless, the editorial results of this subject appeared reasonable. In the hearing, subject $\mathrm{M}$ answered that "the more complex (the description of an item), the higher was the level I intended to place the item, but the positions were really dubious."

\subsubsection{When the definition of description on the space for effects on me is ambiguous}

As described in Sect. 7.1, some subjects were confused when describe on the space for effects on me.

\section{Discussion}

In this section, we verify that ethical considerations promote creativity and discuss possible updates to Dfrome and extensions of ethical design theory that would reconcile the above-noted limitations.

\subsection{Verification that ethics support creativity}

Ideally, an investigation should yield pattern $\mathrm{i}$, which verifies that creativity is inspired by ethics (i.e., Hypothesis 1). First of all, we discuss how its mechanism was realized as the horizontal and vertical elements (i.e., Hypothesis 1.1). Pattern i was achieved via two approaches: the semantic route realized by the recommended scenarios, which trigger ethics-based creativity, and the spatial route realized by the representation field, in which the mapped results in the perspectives trigger the ethics-based creativity. The semantic route was achieved by subjects $\mathrm{A}$ and $\mathrm{D}$; the spatial route was achieved by subject $M$.

In the semantic case, we could confirm that there were corresponding experiences to the two elements as is shown in Sect. 7.2.1. Then, it is notable that subject A could unexpectedly generate a positive application through a risk scenario. This case is considered to be an advanced one but can be explained by studying the two elements in Fig. 18, too. Firstly, the general process of obtaining a novel application from a risk scenario can be considered as is shown in Fig. 6. Differently from Fig. 18, a designer in this scenario envisages positive effects related to risk. Then, the Dfromebrowser result of subject A was more complex, because text (words) was submitted to the browser as an input. The process of this case is demonstrated in Fig. 7. This process is rather an applied process with an additional tree-like description of the research theme developed in the subject's mind. However, the fundamental of the process does not change: doubting from the ethics level promotes us to 
Fig. 6 General process of generating a novel application from a risk scenario

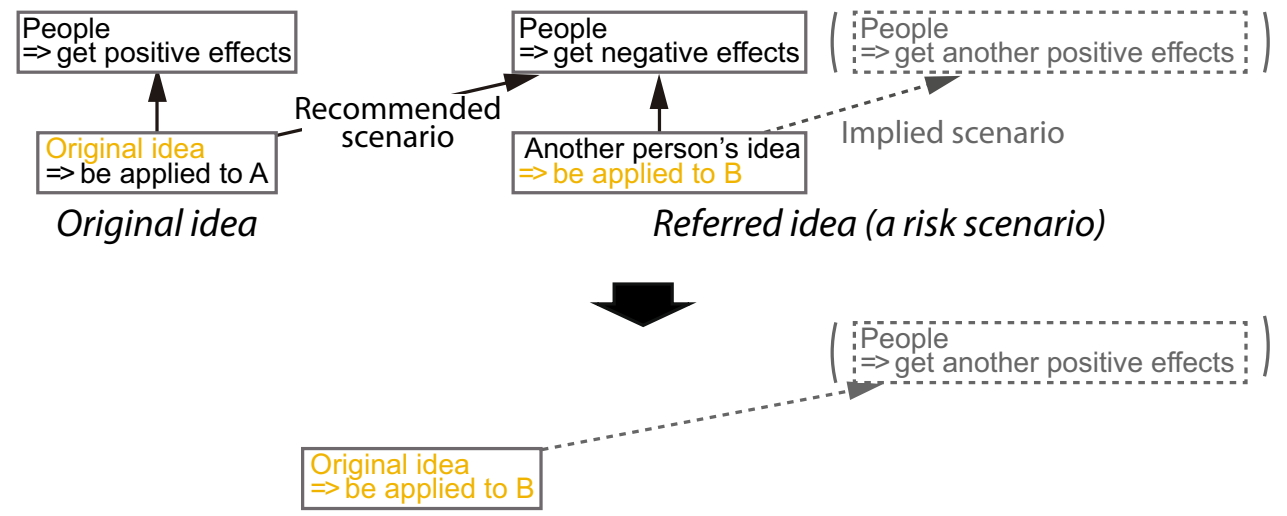

Generated application

Fig. 7 The generation of novel ideas from a risk scenario in the Dfrome browser: a special case
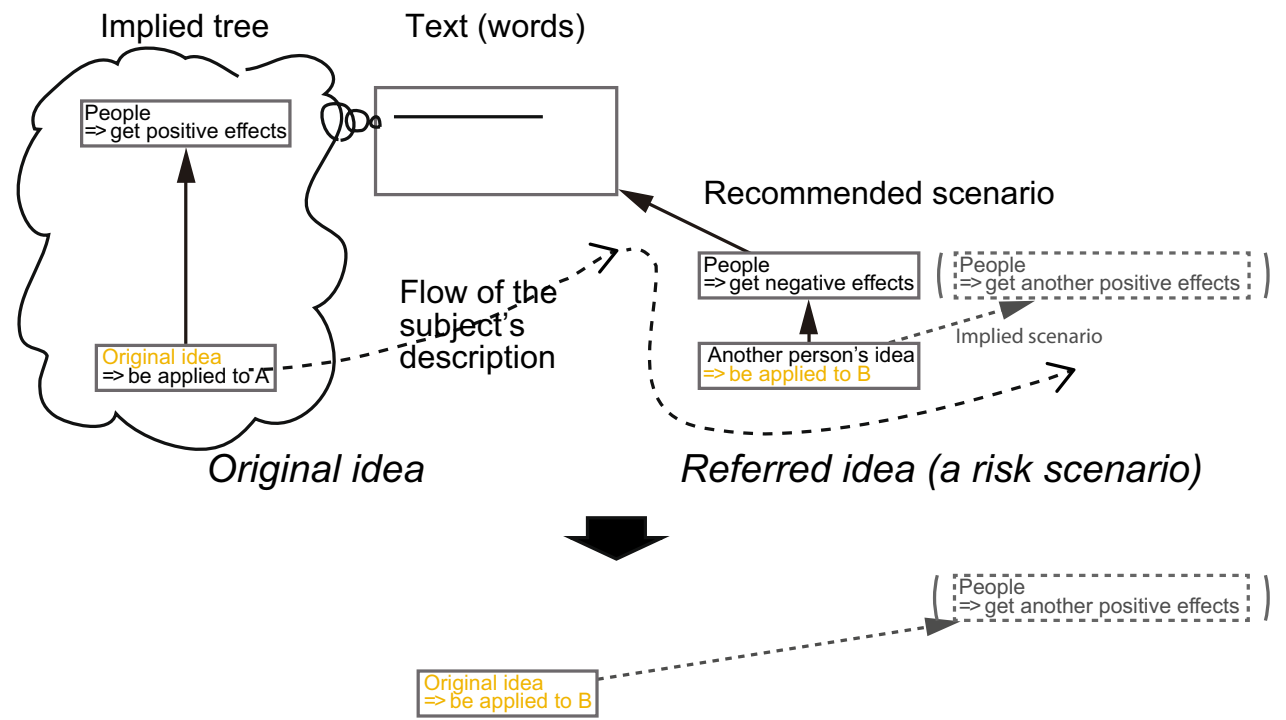

Generated application become aware of a wider conceptual space for design solutions. In this way, the observed phenomena were explainable by the two elements.

The spatial route is realized via the Dfrome editor and the mechanism can be explained by the two elements as is shown in Sect. 7.2.1, too. During this process, the generation of a novel design solution is separated from the cognizance of a wider conceptual space. Thus, a designer can become aware of a wider space without generating a novel solution. It can be expected that novel design solutions are then found from the scenario path recommendations of Dfrome's editor, similar to the above-mentioned semantic case. In relation to these points, Dfrome were partially successful to support practicing the ethical design theory (i.e., Hypothesis 3). For example, the subjects of the user studies tended to ignore the recommendations of the Dfrome editor, probably because they mainly intended to edit their own ideas. We surmise that the motivation and incentives to generate new ideas were insufficient for some subjects, because idea generation was not part of the experimental design (see Sects. 6.3, 6.4, 7.3).

Regarding the sub-subhypothesis that we can obtain the widest conceptual space when we introduce ethics (i.e., Hypothesis 1.1.1), we obtained supportive results . As is discussed in Sect. 7.3.1, subjects were able to connect their own ideas to ethical values, such as privacy, military usage 
and its restriction whose original scenario was connected to peace issues, and safety. If the scenario paths connected to these values were consistent, the originality of their ideas would be higher, because these values are rarely dealt with in the substantial engineering field.

Additionally, there are results of the creativity support effect without ethics as pattern ii, as listed in Table 9. However, if we can doubt these results from the ethics level, the conceptual spaces are to be widened further. For example, regarding subject B's consideration to improve the motivation of aerodynamic engineers, we can further reconsider why such improvement is important for society. That is, it might improve efficiency to realize economic growth, improve safety in global transportation, improve freedom by the self-realization of engineers, etc. Therefore, we can notice a wider conceptual space of design solution of such data analysis. We can also consider an idea that optimizes aerodynamic performance of an aircraft to maximize the economic growth realized by the transportation system, which requires optimization of materials, production lines, maintenance costs, etc. simultaneously. Similarly, we can obtain wider conceptual spaces regarding the Pattern ii cases when we introduce ethics.

We next discuss the sub-subhypothesis regarding the difficulty of doubting from the ethics level (i.e., Hypothesis 1.1.2): we considered that the higher the initial target level was, the easier it was for the designer to doubt from the ethics level. However, as is discussed in Sect. 7.3.1, it was confirmed that the semantic factor (i.e., topics of the subject) was more influential than this syntactic factor. Thus, after improving our knowledge base, we will need to evaluate the syntactic factor again.

We next evaluate the hypothesis that practicing ethical design theory promotes ethical design (i.e., Hypothesis 2). As is discussed in Sect. 7.3, one of the key factors to cause the creative ethical design in pattern $i$ includes the method of providing information and the visualized image of perspectives. These points were clearly provided by the ethical design theory and subjects in the stakeholder impact study were more likely to experience pattern i than Google when Dfrome supported practicing the ethical design theory as is shown in Sect. 7.3.1. Therefore, the ethical design theory succeeded in promoting ethical designs.

Then, we evaluate the hypothesis that Dfrome supports practicing ethical design theory (i.e., Hypothesis 3 ). As mentioned above, this point was partially confirmed: it failed in changing subjects' intentions by Dfrome's editor. However, Dfrome succeeded in supporting the subjects to utilize scenarios recommended by Dfrome's browser and visualized image of perspectives presented by Dfrome's editor. Furthermore, Dfrome was more likely to cause Pattern i than Google as is shown in Sect. 7.3.1. Additionally, Dfrome could be used by professional subjects at any place where users could connect to the internet through a web browser on their own computer. Therefore, Dfrome analysis was more convenient than seeking advice from specialists, such as science interpreters, ethicists, and engineering professors, who are not always physically present and who handle one theme at one time. Furthermore, we could confirm that even professional AI engineers can edit their ideas on Dfrome. Because we have confirmed that ethical scenarios could be edited on Dfrome with an AI ethics/AI expert and a public-law expert Sekiguchi and Hori (2018a), the ideas accumulated in Dfrome's knowledge base cover the gamut from technical to ethical discourses, implying that Dfrome is potentially much more comprehensive than any professional investigator. This universal feature of Dfrome also ensures a worldwide dissemination of the ethics-based effect on creativity.

These results support the claim that criticism still supports creative activities, even with an ethical perspective. Furthermore, it is also supported that ethics can make the creativity support effect by criticism greatest.

Additionally, we can discuss the viewpoint of whether the ethical design theory and Dfrome can solve the problem suggested by the FBS diagram and modeler (i.e., it was not easy to express subjective affairs, and many designers feel it difficult to symbolize functions as subjective affairs). Then, the ethical design theory and Dfrome were observed to solve this problem and succeeded in becoming more practical than the FBS diagram and modeler. In fact, the ethical design theory and Dfrome were able to support subjects to intuitively express subjective affairs in the space for personal concerns as described in Sect. 7. The reason why subjects were able to understand the distinction between personal reasons and ideas in the hierarchical representation was that personal reasons correspond to the background of their own themes, which always demand to be explained in an academic paper, business documents, etc. Furthermore, ideas in the hierarchical representation corresponds to what they actually do as their work. However, the definition of the description in the space for effects on me was sometimes confused. To more clearly explain the distinction between the hierarchical representation and the effects on me more is a topic of future works.

Finally, we discuss implication of the above-mentioned verification. Firstly, validity of the ethical design theory 
Fig. 8 Four dimensional representation of design from the ethics level

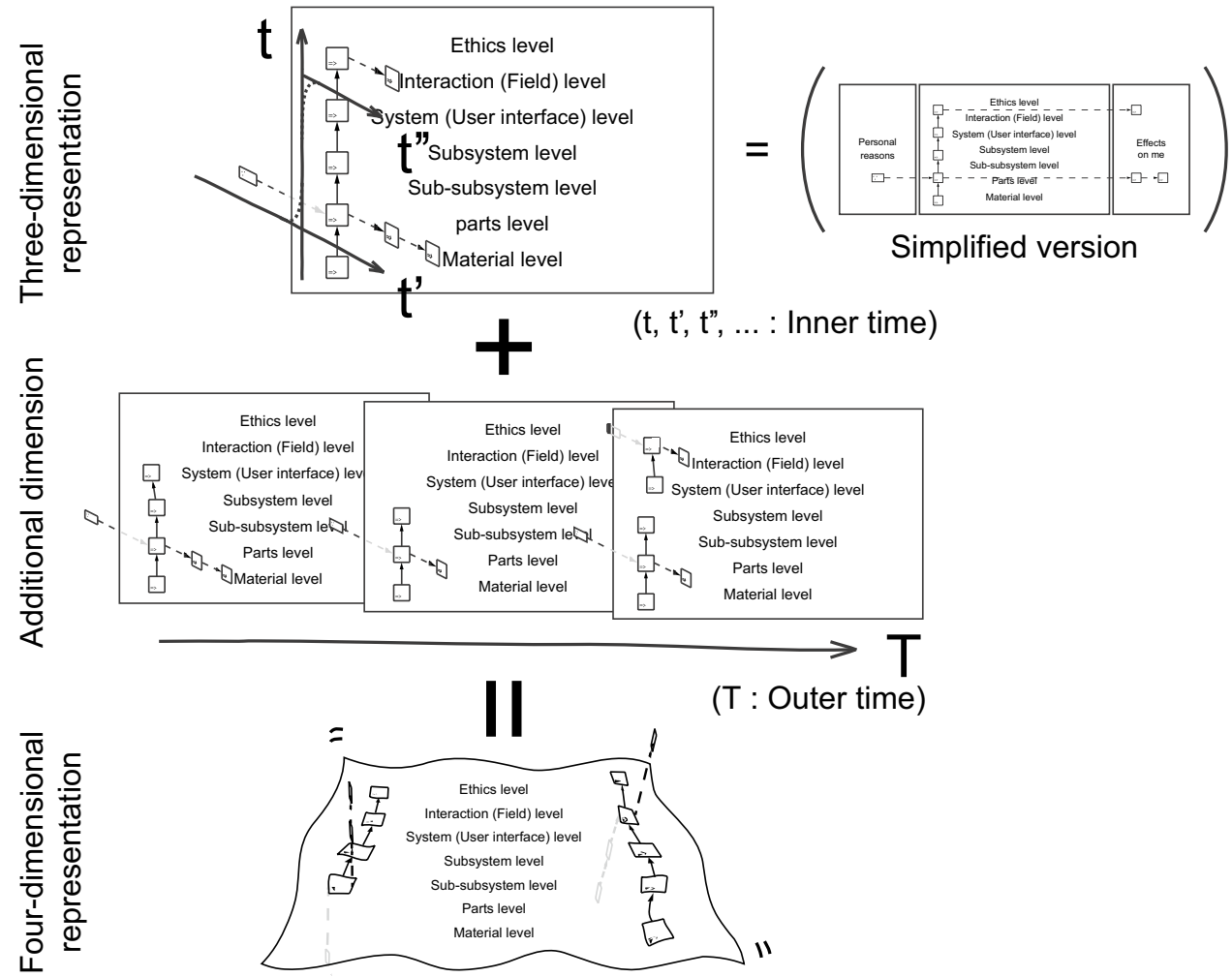

itself could be supported because we could confirm that we can explain and predict the existence of creativity support effect of ethics and its mechanism using the ethical design theory, especially Hypotheses 1, 1.1, 1.1.1 and 2. Then, the ethics-based creativity support may be used to reconcile ethics with science and technology, because it confirms that ethics need not stifle innovation. Rather, it improves the sustainability of research and development, because designers can realize original work without losing accountability for their projects. The empirical nature of the current verification is also significant, because engineering is an intrinsically empirical field. The tool will also assist businesspersons who prefer to keep being innovative when introducing ethics into their businesses.

\subsection{Extension of ethical design theory and Dfrome}

We then discuss how we can extend the ethical design theory and Dfrome including the knowledge of how to utilize them. First of all, to ensure that pattern i emerges from the study results, we should systematize the conditions that promote the creativity effect of ethics. The results of the present studies provided hypotheses for this systematization. We have already derived the key factors to succeeded and un-succeeded in realizing pattern $i$, so that we can realize this promotion by enhancing the successful key factors in Sect. 7.3. For example, by enriching the contents of Dfrome's knowledge base, we have to improve the organic and dynamic features of Dfrome to more properly deal with user's topics. Another example is that we have to properly set designer's positions in the orthogonal representation of personal concerns. For example, we could alert the designer to the importance of accountability, as observed in the results of subjects $A, D$ and $M$. The designer would then recognize that ethics is useful for generating new ideas. Or more directly, it is probable to disclose the mechanism of the creativity support effect of ethics to users as subject $\mathbf{J}$ mentioned, then the users would introduce ethics to gain a wider conceptual space of design solutions to achieve higher creativity. In future work, these solutions should be verified in further user studies.

To comprehensively explain the observed data, we must extend the ethical design theory. The first requirement is that we should add another dimension to the outer time of design ideas ${ }^{7}$, as shown in Fig. 8. After adding this dimension, the perspectives of ethical design theory would be four-dimensional. The inner time of design ideas is already accommodated in three-dimensional ethical design theory. The outer time is required to explain the recursive relationship mentioned by subject $\mathrm{J}$ and the evolution of business vision mentioned by subject L. When a user, such as a target

\footnotetext{
7 This idea has been described since an essay. Please refer to Kaira Sekiguchi, The sixth and the seventh rules of "design with discourse" for design from the ethics level, January 1, 2011. at http://www.ethic s-level.com.
} 
customer of subject J's startup, installs a device that accumulates data, he or she becomes a medium for the installation and can hence be described as a component (subsystem) of the system. A user who utilizes the accumulated data can then be understood as a beneficiary of the system at the interaction level. In this sense, recursion is described along the inner time dimension. Other relationships, such as feedback retrieval from the data and device-installation updates and/or increases, are described along the outer time dimension. Therefore, only a four-dimensional ethical design theory can fully satisfy the needs of subject J. Then, the business vision evolution can obviously be described in this outer time dimension. Incorporating this dimension into Dfrome is a part of our future task.

The second requirement is the proper positioning of designers' social contexts in the design process. The use of the social context should be properly constrained. Otherwise, the ideas that trigger the ethics-based creativity can be hindered. As a probable solution to this problem, creativity support could be included in the orthogonal representation of personal concerns, as discussed at the beginning of this subsection. In relation to the social context, for example, the human relationships mentioned by subject $\mathrm{K}$ and the responsibility of being accountable mentioned by subjects $\mathrm{J}$ and $\mathrm{M}$ likely relate to the settings of the design subject. Therefore, they can be described in the space of personal reasons. The cost of changing human relationships, highlighted by subject K, can also be described in the space of personal effects. To realize this support in Dfrome, users' personal concerns can be backwardly calculated from the contents described in the hierarchical representation of artifacts, and Dfrome would then use more clearly this information for supporting users to prefer ethical design.

As the third requirement for explaining the observed data, we must clarify the description of ideas in the ethical design theory as a field. Moreover, as mentioned by subject $\mathrm{K}$, the mapping of ideas is more important than drawing arrows. A precise description of items means that we can visually understand the causality between items in this field. To exploit the characteristics of this field, we must define the levels, although feedback from subjects $\mathrm{J}$ and $\mathrm{M}$ revealed that the level definitions were sometimes ambiguous. Generally, level definitions in ethical design theory are pluralistic and inevitably differ among designers. As Dfrome accumulates idea descriptions in its knowledge base, the pluralistic definition of each level is expected to be dynamically obtained. The dynamic definition of levels has already been used in the semi-automatic generation of items by Dfrome (see Fig. 16). Thus, users are expected to understand the positions of their items without referring to a written definition of each level. However, the user studies implied that this support is insufficient, because, even when the mapping succeeded, users were unable to check the correctness of their results without knowing the definitions. One probable solution is a new function that that would allow a user to look over the accumulated items around each Dfrome level, thereby helping them understand the dynamic nature of the definition as knowledge accumulates. Furthermore, similar functions would be useful for solving problem of ambiguous definitions of the description of effects.

Finally, the extended version of ethical design theory demonstrated a cyclic relation among theory, experimental equipment, and observed data. Without this cycle, the theory would be an empty husk. As the theory implemented in Dfrome becomes more practical by reconciling with observed data, the usefulness of the evolving knowledge base will improve.

\section{Conclusions and future work}

Ethics are often regarded as barriers to innovation. However, practitioners of ethical design theory who use Dfrome are expected to gain creative activity, (A) by reconsidering their design ideas from ethical perspectives, and (B) by becoming aware of a wider conceptual space. User studies confirmed that both processes are feasible.

We organized the key factors of success when experiencing the creativity support effect of ethics (e.g., the way to provide information, the design of designers' personal concerns, and the visualization of design from the ethics level), and discussed how to utilize them to successfully emerge in design activities. We also updated the ethical design theory to better explain the obtained observation data, which revealed limitations in the experimental version of the theory. The suggested solutions included adding another temporal axis to the originally three-dimensional theory, imposing the social contexts of each user as constraints on further creativity support, and clarifying the perspective of ethical design theory as a representation field.

In future works, we plan to update Dfrome to correspond to the extended version of ethical design theory. We will also check the theory in additional user studies and update it in consideration of the cyclic relation among theory, 
experimental equipment, and observed data. By collaborating with ethicists, policymakers, economists, and other experts, we plan to enrich the contents of Dfrome by accumulating new data of their specialties and to discuss the validity of ethical design theory. Finally, we plan to establish ethical design science as an organic discipline for systematically designing a better world.

Acknowledgements This work was partially supported by JSPS KAKENHI Grant number JP18K18434.

Open Access This article is licensed under a Creative Commons Attribution 4.0 International License, which permits use, sharing, adaptation, distribution and reproduction in any medium or format, as long as you give appropriate credit to the original author(s) and the source, provide a link to the Creative Commons licence, and indicate if changes were made. The images or other third party material in this article are included in the article's Creative Commons licence, unless indicated otherwise in a credit line to the material. If material is not included in the article's Creative Commons licence and your intended use is not permitted by statutory regulation or exceeds the permitted use, you will need to obtain permission directly from the copyright holder. To view a copy of this licence, visit http://creativecommons.org/licenses/by/4.0/.

\section{Appendix}

See Figs. 9, 10, 11, 12, 13, 14, 15, 16, 17, 18, 19, 20, 21, 22 , $23,24,25,26,27,28$ and 29

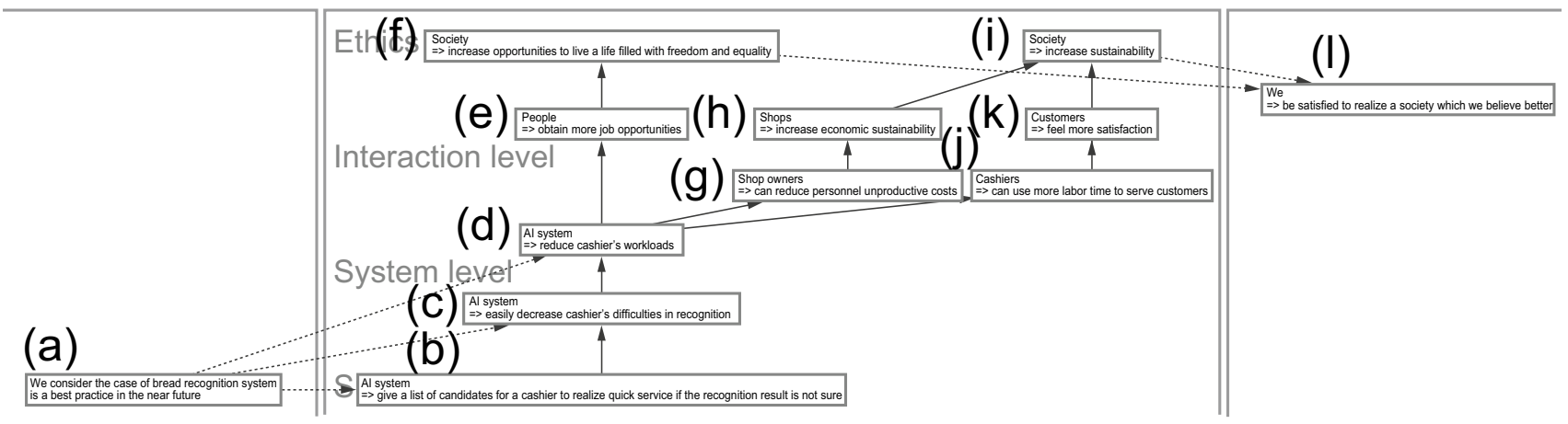

Fig. 9 An image of a revised version of design of an AI system that supports shop cashiers whose descriptions include: (a) $\because$ we consider the case of bread recognition system is a best practice in the near future, (b) AI system => give a list of candidates for a cashier to realize quick service if the recognition result is not sure, (c) AI system $=>$ easily decrease cashiers' difficulties in recognition, (d) AI system $=>$ reduce cashier's workloads, (e) people $=>$ obtain more job opportunities, (f) society $=>$ increase opportunities to live a life filled with free- dom and equality, (g) Shop owners $=>$ can reduce personnel unproductive costs, (h) Shops => increase economic sustainability, (i) society $=>$ increase sustainability, $(\mathrm{j})$ cashiers $=>$ can use more labor time to serve customers, (k) customers $=>$ feel more satisfaction, (l) We $=>$ be satisfied to realize a society which we believe better (see IEEE 2016, 2017; Oka and Morimoto 2015, 2016). This image is from Sekiguchi and Hori (2018a). Some misspelled words were fixed by authors 


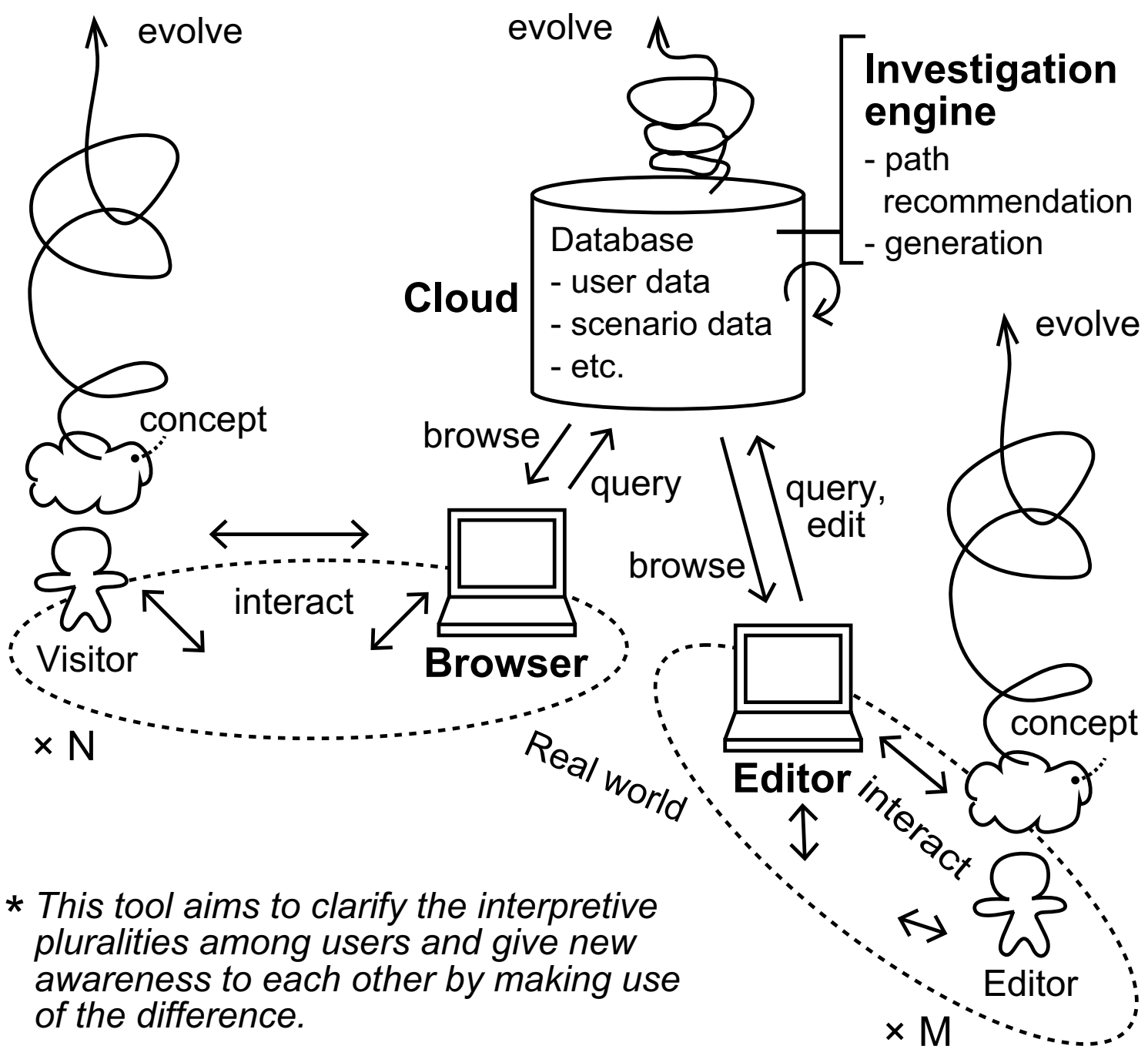

Fig. 10 Overview of the organic and dynamic tool, Dfrome Sekiguchi and Hori (2018b). In the user studies presented in this paper, semi-automatic generation functions were added to the investigation engine 
Fig. 11 Example of semiautomatic generation process of Dfrome
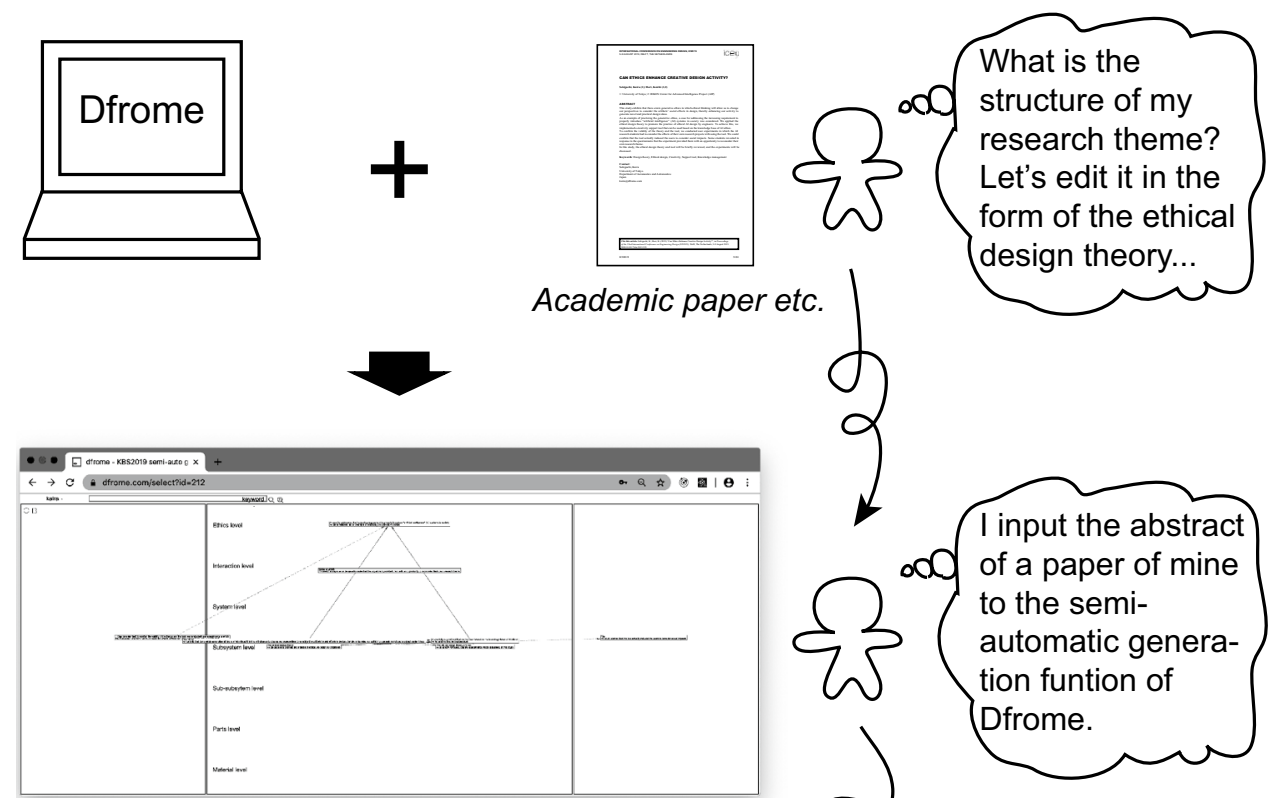

A result of the semi-automatic generation

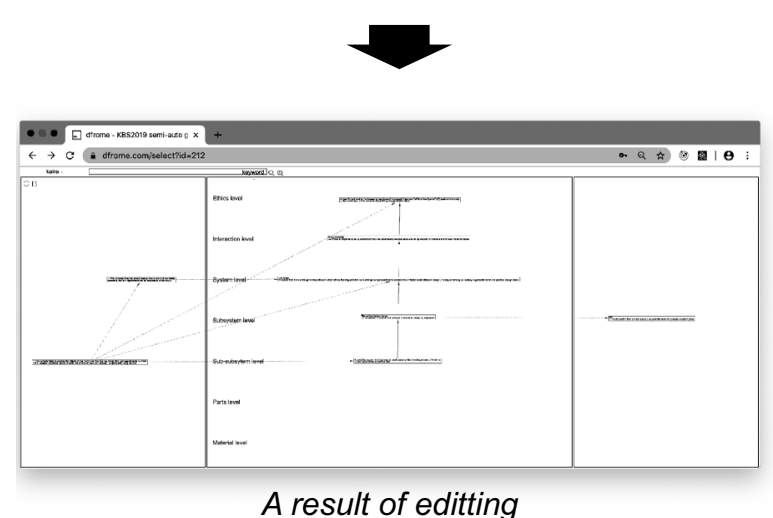

A result of editting 


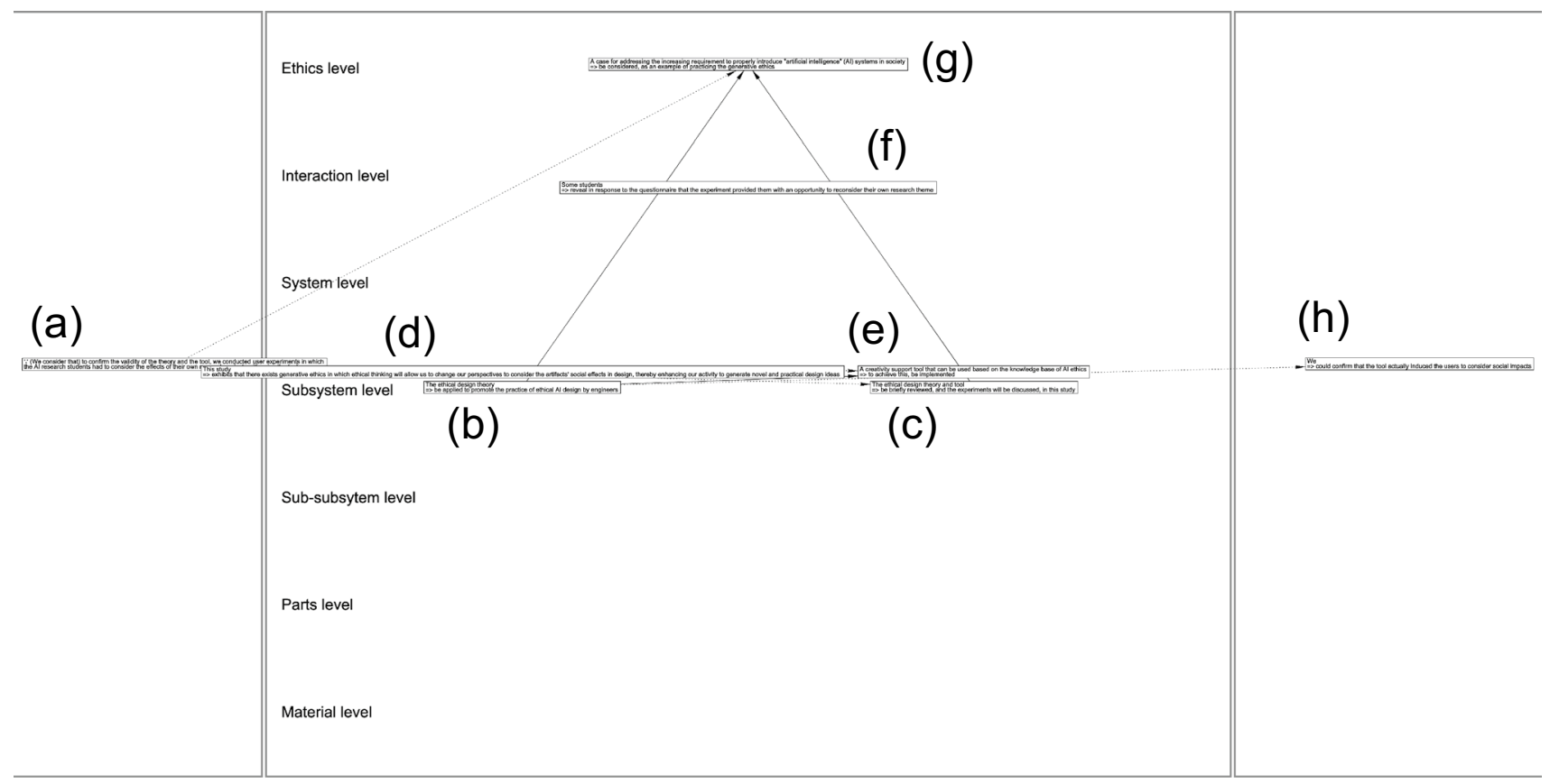

Fig. 12 Example of semi-automatic generation: (a) $\because$ (we consider that) to confirm the validity of the theory and the tool, we conducted user experiments in which the AI research students had to consider the effects of their own research projects with using the tool; (b) the ethical design theory $=>$ be applied to promote the practice of ethical AI design by engineers; (c) the ethical design theory and tool $=>$ be briefly reviewed, and the experiments will be discussed, in this study; (d) this study $=>$ exhibits that there exists generative ethics in which ethical thinking will allow us to change our perspectives to consider the artifacts' social effects in design, thereby enhancing our activ- ity to generate novel and practical design ideas; (e) a creativity support tool that can be used based on the knowledge base of AI ethics $=>$ to achieve this, be implemented; (f) some students $=>$ reveal in response to the questionnaire that the experiment provided them with an opportunity to reconsider their own research theme; $(\mathrm{g})$ a case for addressing the increasing requirement to properly introduce "artificial intelligence" (AI) systems in society $=>$ be considered, as an example of practicing the generative ethics; ( $h$ ) we $=>$ could confirm that the tool actually induced the users to consider social impacts (see Sekiguchi and Hori 2019) 


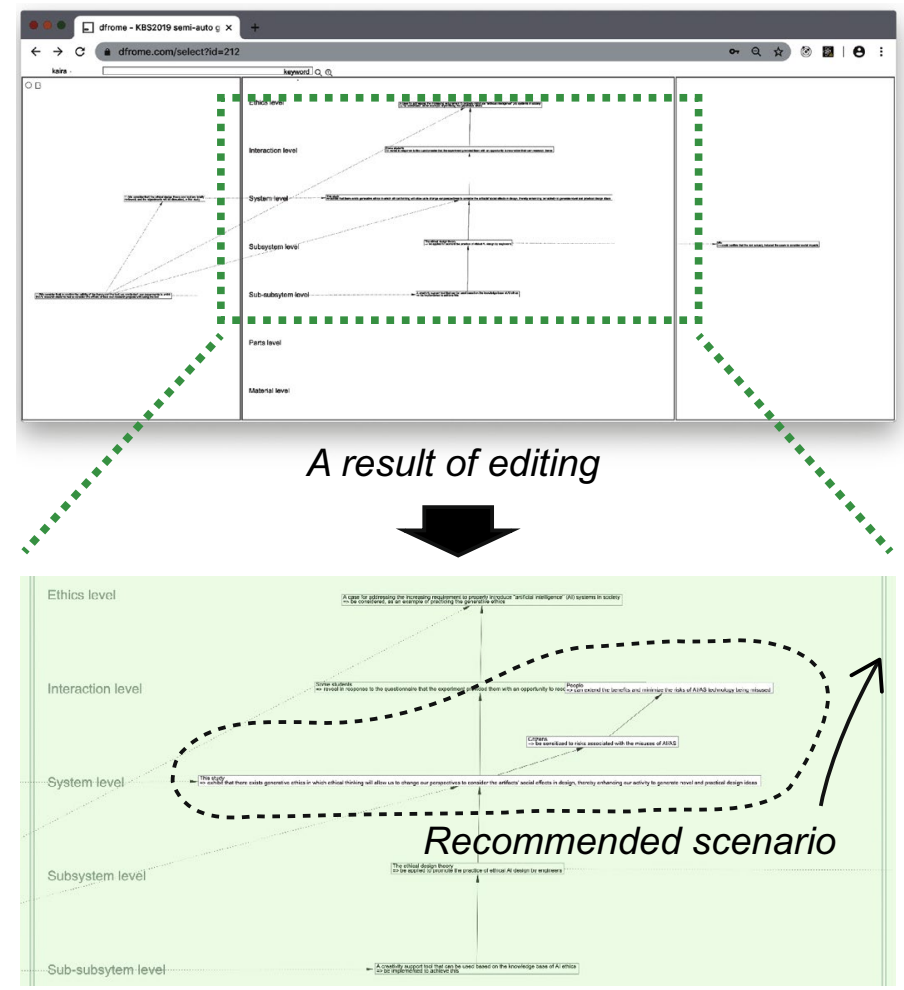

A result of upward recommandation
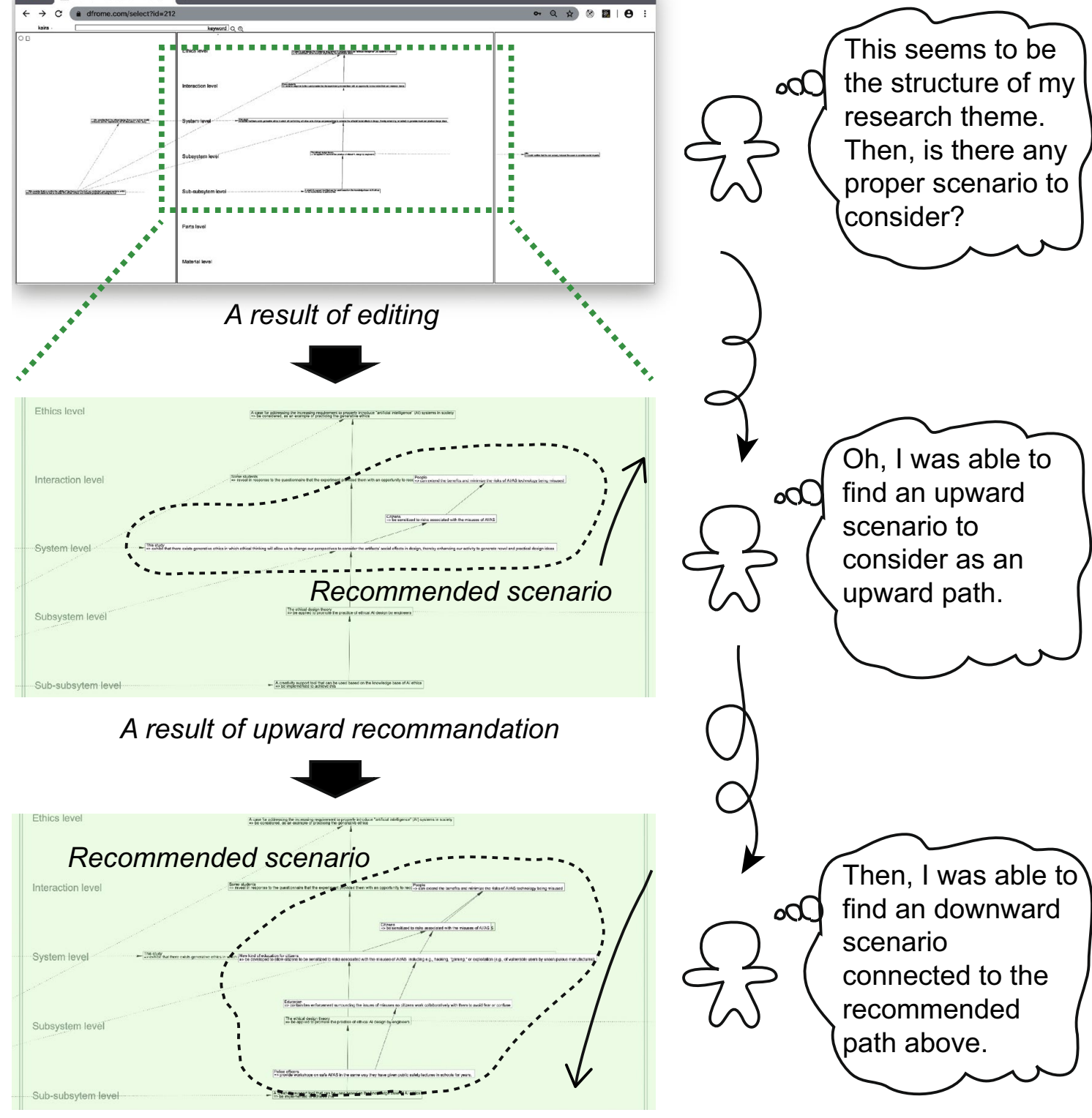

A result of downward recommendation

Fig. 13 Example of using the scenario path recommendation in Dfrome's editor 


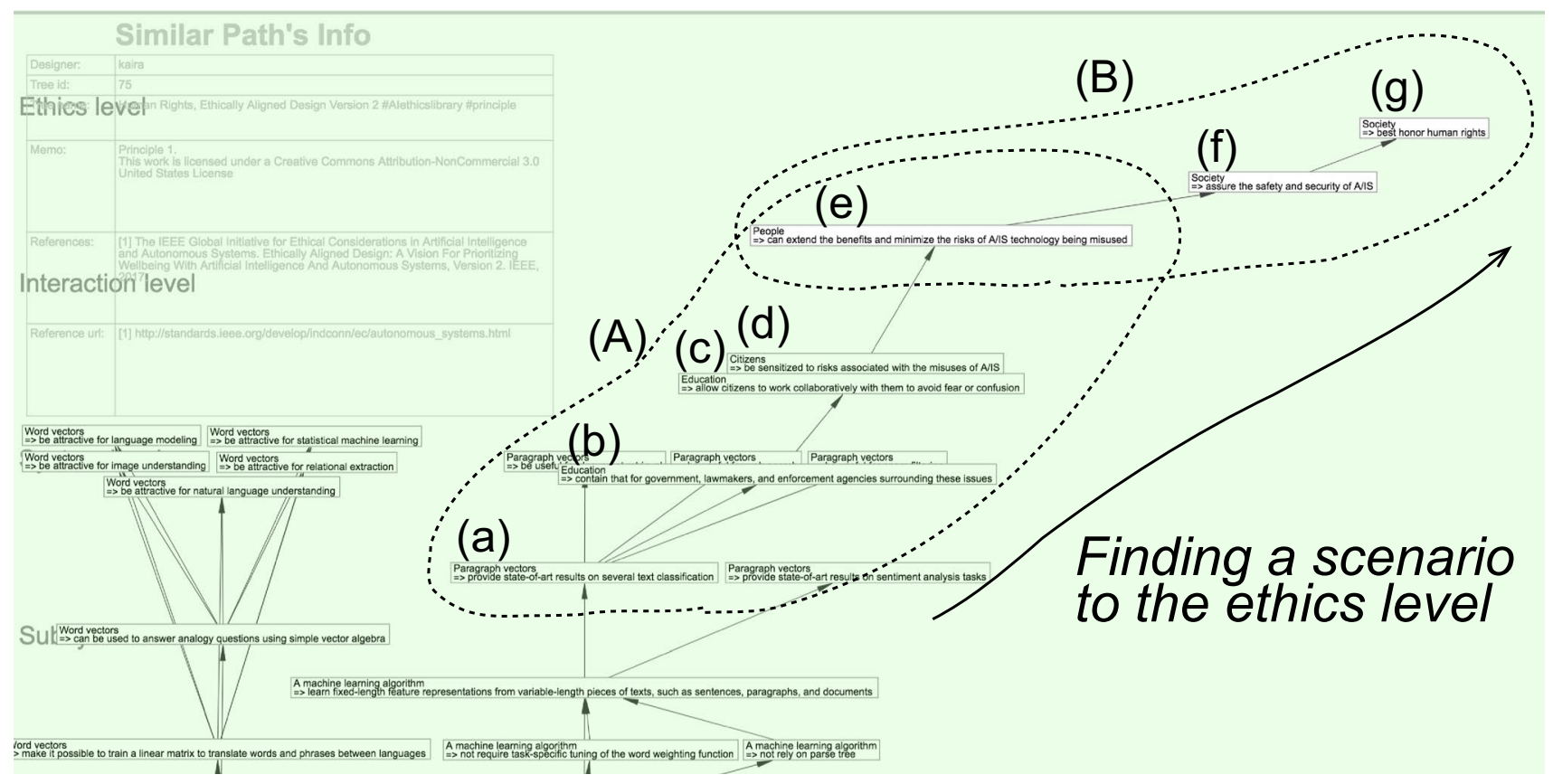

Fig. 14 Example of path recommendation to the ethics level: (a) paragraph vectors $=>$ provide state-of-art results on several text classification; (b) education $=>$ contain that for government, lawmakers, and enforcement agencies surrounding these issues; (c) education $=>$ allow citizens to work collaboratively with them to avoid fear or confusion; (d) citizens $=>$ be sensitized to risks associated with the misuses of A/IS; (e) people $=>$ can extend the benefits and minimize the risks of A/IS technology being misused; (f) society $=>$ assure the safety and security of A/IS; (g) Society => best honor human rights (Le and Mikolov 2014; IEEE 2017); path(A)'s rank is 2; (B)'s rank is 7 (Sekiguchi and Hori 2018a) 


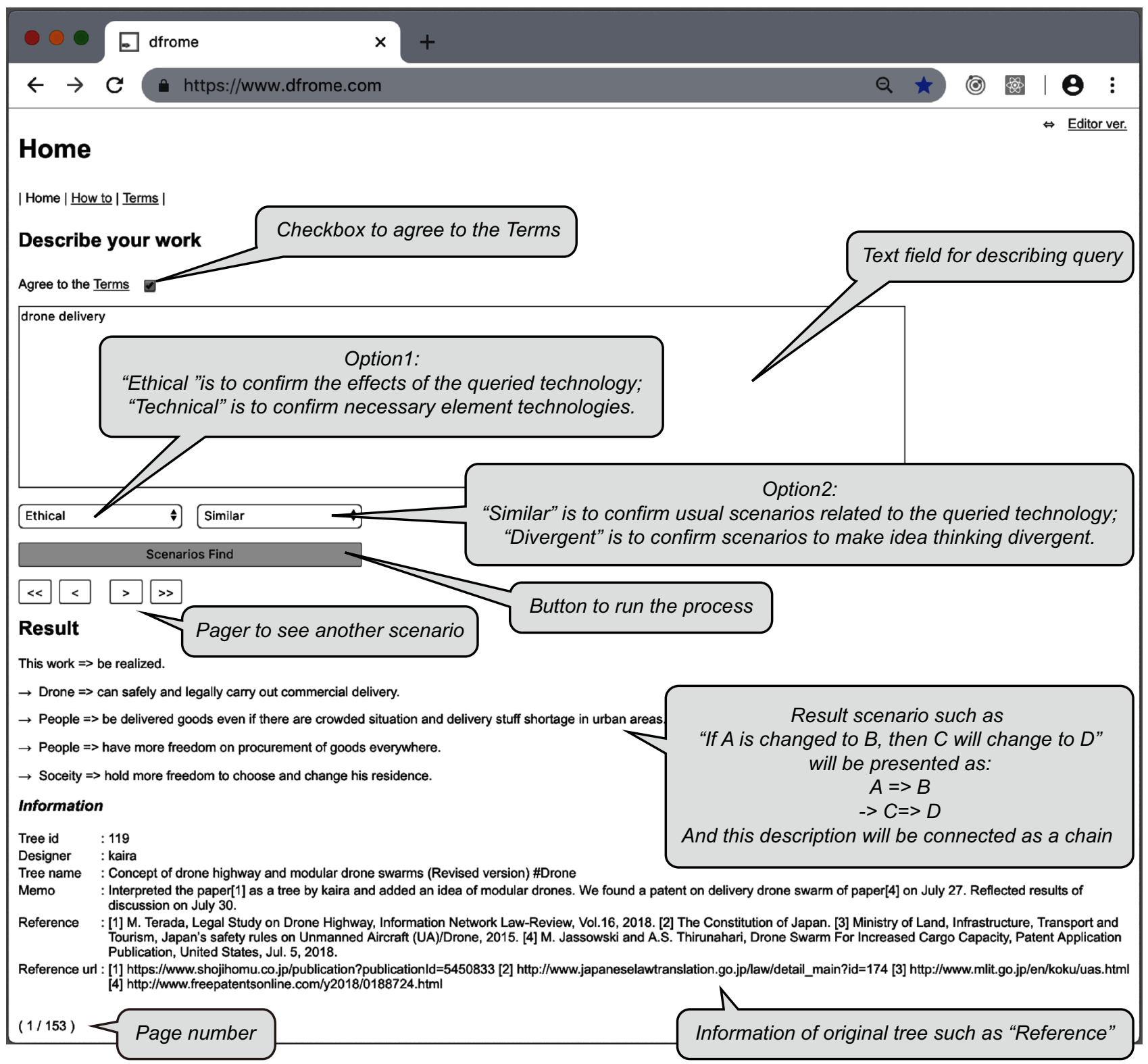

Fig. 15 Overview of the Dfrome's browser (Sekiguchi and Hori 2019). A misspelled word was fixed 


\section{(1) Parse an input text and make items}

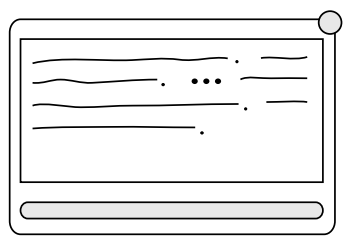

Users are to open a window from the context menu of Dfrome, input a text and click the execute button.
Sentence 1: $\quad \mathrm{S}_{\mathrm{s} 1}+\mathrm{V}_{\mathrm{s} 1}+\sim$.

4

Sentence N: $\mathrm{S}_{\mathrm{sN}}+\mathrm{V}_{\mathrm{sN}}+\sim$.

Dfrome parses a text into sentences $(N)$ and parses the syntax of each sentence ${ }^{\star 1}$. $\begin{array}{ll}\text { Item 1: } & \because \mathrm{S}_{\mathrm{s} 1}+ \\ \mathrm{V}_{\mathrm{s} 1}+\sim\end{array}$ and $\begin{aligned} & \mathrm{S}_{\mathrm{s} 1} \\ & \Rightarrow \mathrm{V}_{\mathrm{s} 1}{ }^{\prime}+\sim\end{aligned}$

$\begin{array}{ll}\text { Item N: } & \because \mathrm{S}_{s N}+ \\ \mathrm{V}_{s N}+\sim\end{array}$ and $\begin{aligned} & \mathrm{S}_{s N} \\ & \Rightarrow \mathrm{V}_{s N^{\prime}}+\sim\end{aligned}$

Dfrome makes the two types of items for each sentence:

" $S_{s i}+V_{s i}+\sim$ " and "S $S_{s i}=>V_{s i}$ ' $\sim$ " *2

\section{(2) Position the generated items in the directions for causality}

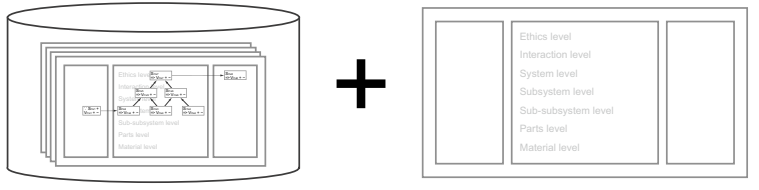

Dfrome has many active and public trees in the knowledge base and Dfrome has the representation field.

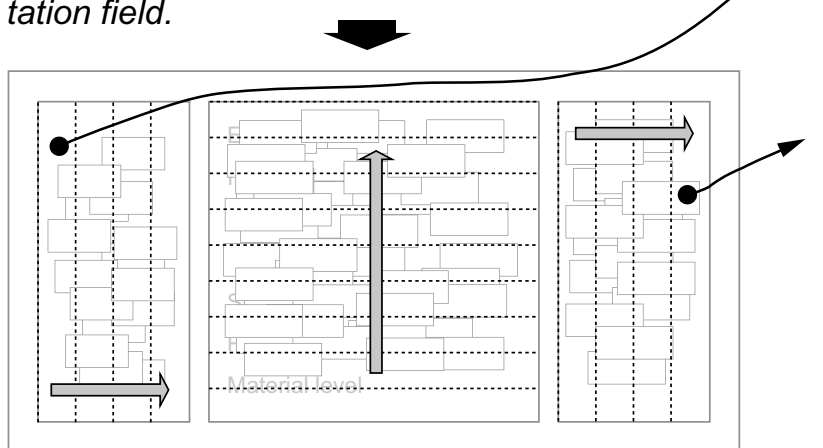

Several items exist in each mesh (M) divided at a constant pitch in a direction representing a causal relationship (gray arrows) (text in each item is omitted here for simplicity).

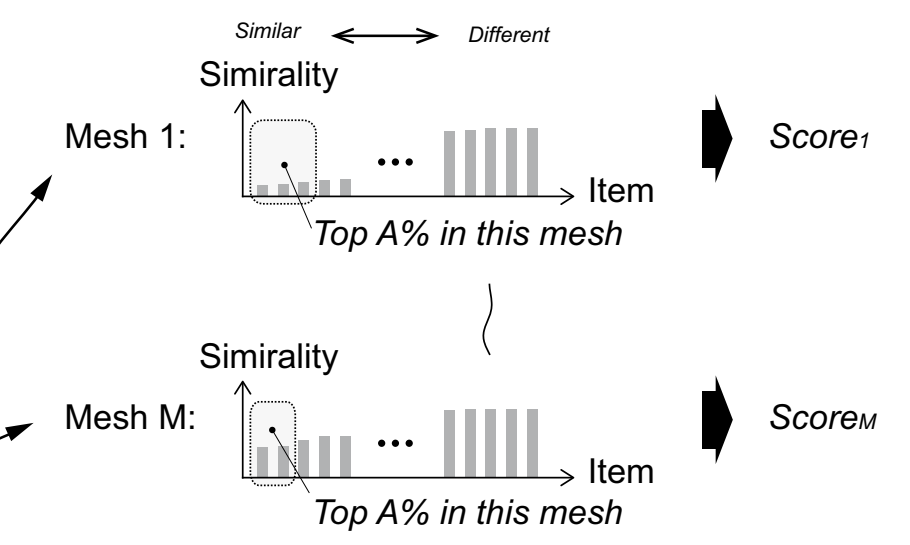

Dfrome calculates similarities ${ }^{* 3}$ between a candidate item and the items in each mesh, orders the values of the similarities and calculates a score by averaging top $A \%$ of similarities in each mesh.

Dfrome selects the mesh with the best score (minimum one) from the calculated scores to select where the candidate item will be positioned.

\section{(3) Layout items by ordering in the directions for comprehensiveness}

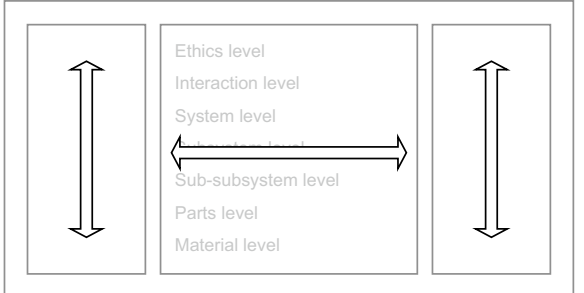

Dfrome layouts each item at the position of the mesh detected above ${ }^{* 4}$. When more than two items exist in a mesh, these items will be arranged at equal pitches in the direction (white arrows) orthogonal to the direction for causality.

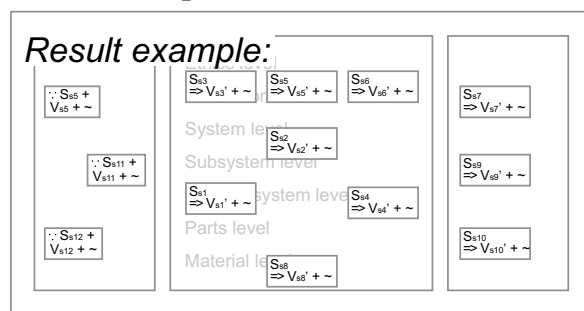

*1 Sentences with some verbs (apply, develop, realize, etc.) were transformed into the passive voice.

*2 " $V$ si'" signifies that its root verb was lemmatized and lowered.

${ }^{* 3}$ simirality $=1-\cos (v($ candidate item $), v($ item in mesh $))+k_{\text {space }}$

Here, "cos" means to calculate a cosine simirality between two vectors. A vector for sentence $x$ is signified as " $v(x)$ " which is defined in a document embedding space. As the sentence " $x$ ", the whole sentence is used for the space for Personal concerns, and subjective is used for the other two spaces: the Hierarchical representation of artifacts and the space for Effects on me. "kspace" is heuristically defined for each space.

*4 To clarify the subjective nature of the personal reason items, "(We consider that)" is added as " $\because$ (We consider that) $S_{s i}+V_{s i}+\sim$ "during this step.

Fig. 16 Basic idea of calculation process of the mapping items of the semi-automatic generation 


\section{(1) Get the data on the representation field}

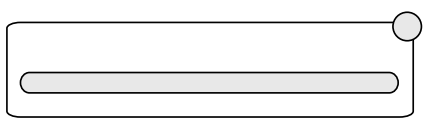

Users are to open a window from the context menu of Dfrome and click the execute button. Then, Dfrome gets the data on the representation field.

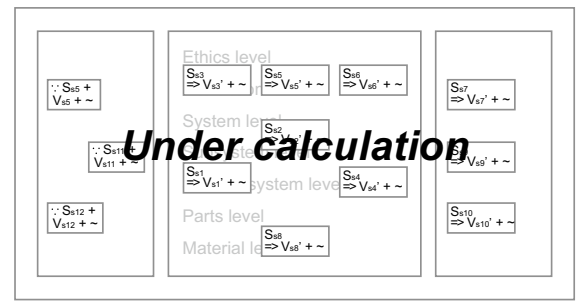

\section{(2) Take all the potential arrows into account}

Dfrome takes all the potential arrows among items ${ }^{* 1}$ on the representation field into account: Logically drawable cases by the ethical design theory are as follows (text in each item is omitted here for simplicity):

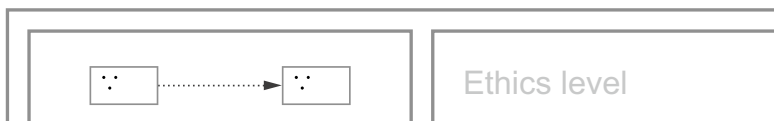

Case 1: Both root and target items are in the personal-concerns space $(\mathrm{P})$ and the target item lies to the right of the root item.

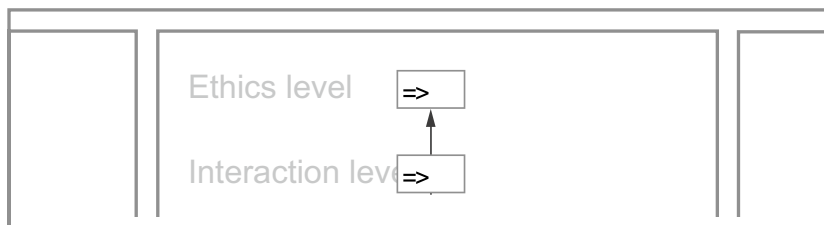

Case 3: Both items are in $\mathrm{H}$ and target item lies above the root item.

\section{s level}
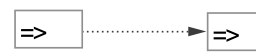

Case 5: Both items are in $E$ and the target item lies to the right of the root item.

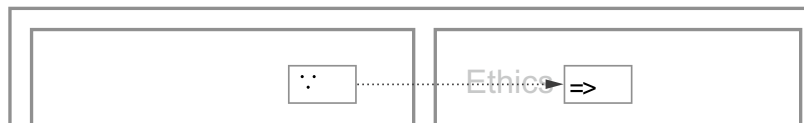

Case 2: The root item is in $\mathrm{P}$ and the target item is in the hierarchical repre 7 sentation of artifacts $(\mathrm{H})$.

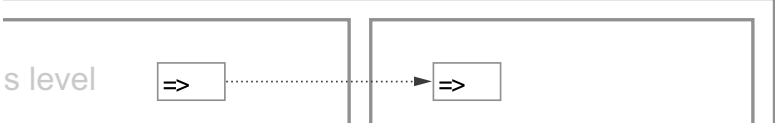

Case 4: The root item is in $\mathrm{H}$ and the target item is in the effects on me space (E).

\section{(3) Calculate whether a similar arrow exist in knowledge base}

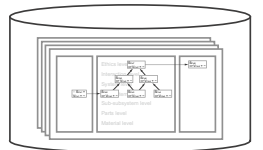

Dfrome has many active and public trees in the knowledge base.

Dfrome calculates each similality total $^{* 2}$ between a potential arrow and active and public arrows in the knowledge base. if any similarity is under a certain threshold, Dfrome draw a new arrow.
*1 The item each arrow starts is called 'root item' and that each arrow end is called 'target item'.
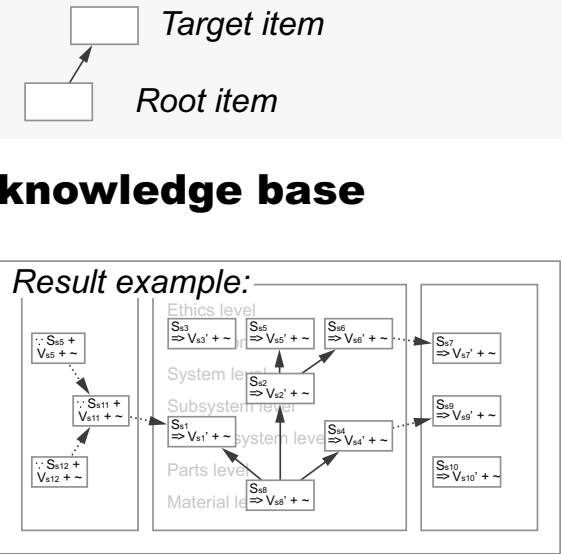

*2 similarity root $\quad=1-\cos (v($ potential root item $), v($ existing root item $))$

similaritytarget $\quad=1-\cos (v($ potential target item $), v($ existing target item) $)$

similarity total $=$ simirality ${ }_{\text {root }}$ * simirality target

Here, "cos" means to calculate a cosine similarity between two vectors. A vector for sentence $x$ is signified as " $v(x)$ " which is defined in a document embedding space. As the sentence " $x$ ", the whole sentence is used. This calcularion is omitted (1) where an arrow have already been existed or (2) the types of the two calculated arrows are different. These "types" are what is definedin the program: (a) arrow with the root and target items are in $P$, (b) that with the root and target items are in E, and (c) the other cases.

Fig. 17 Basic calculation process of connecting items by the arrows of the semi-automatic generation 

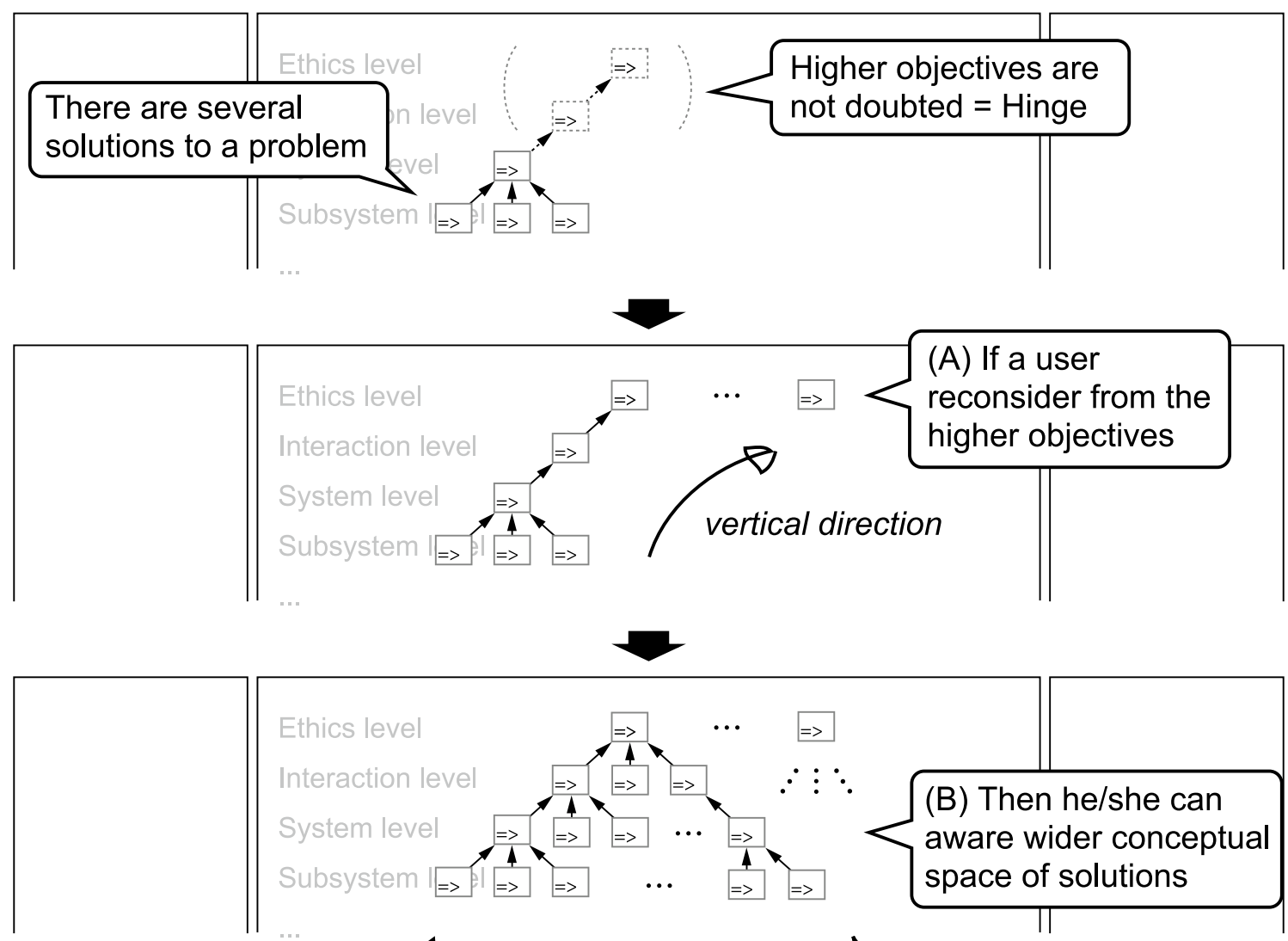

horizontal direction

Fig. 18 Mechanism of creativity support effect by ethics

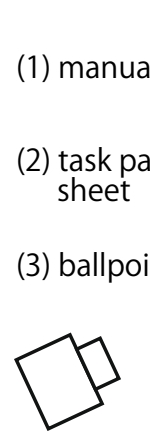

(4) camera
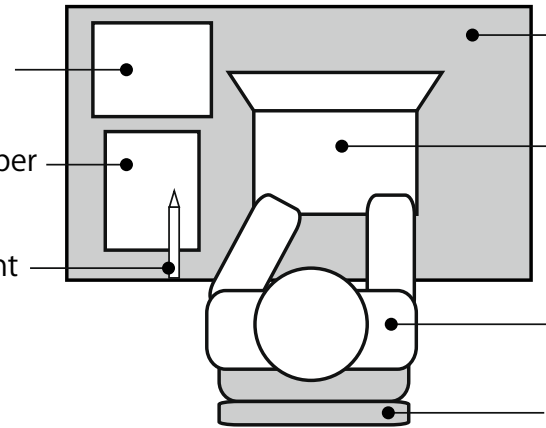

(8) chair

Experimental environment

(5) desk which runs
(7) subject

(6) laptop on Dfrome

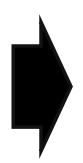

(6)

(5)

(1)

(2)

(3)

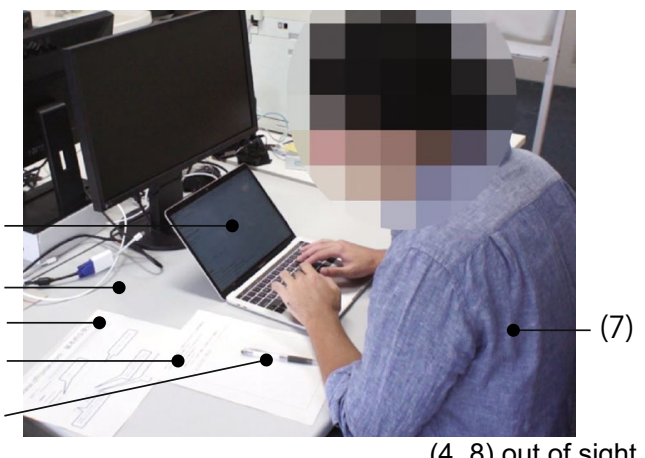

Actual image

$(4,8)$ out of sight

Fig. 19 Schematic and photograph of the experimental environment (Sekiguchi and Hori 2019) 


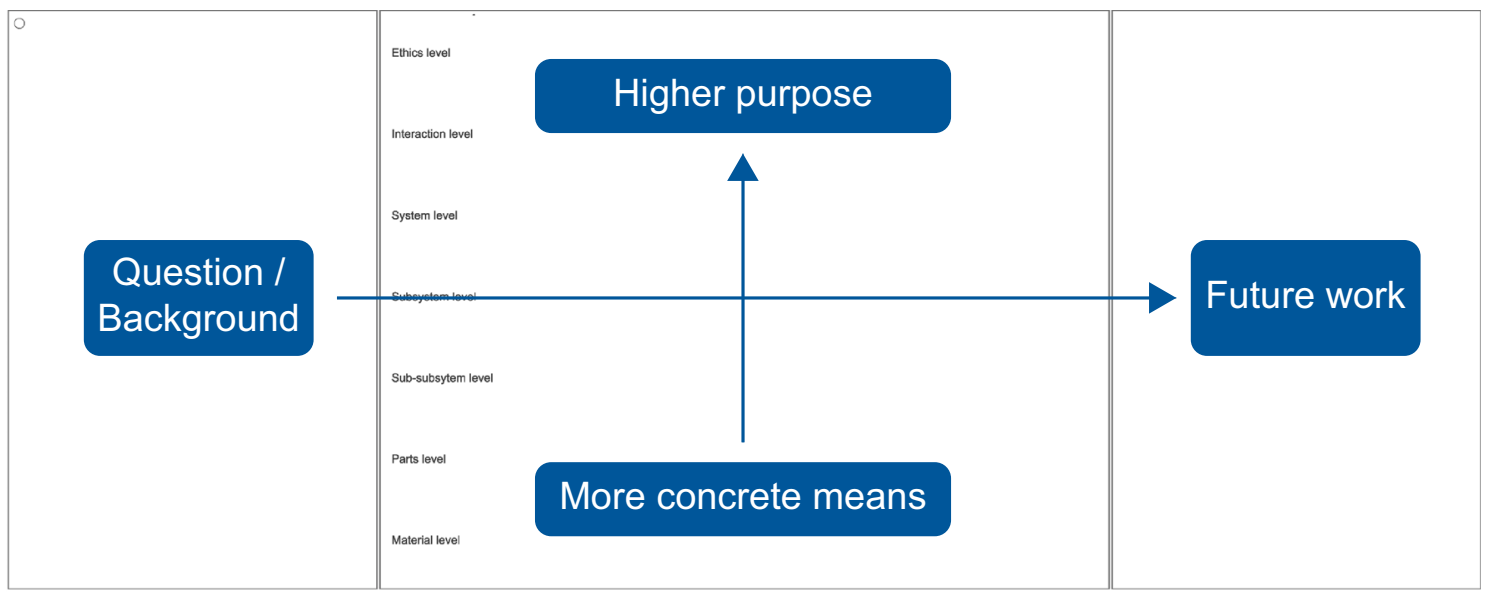

Fig. 20 How to see the Dfrom representation 


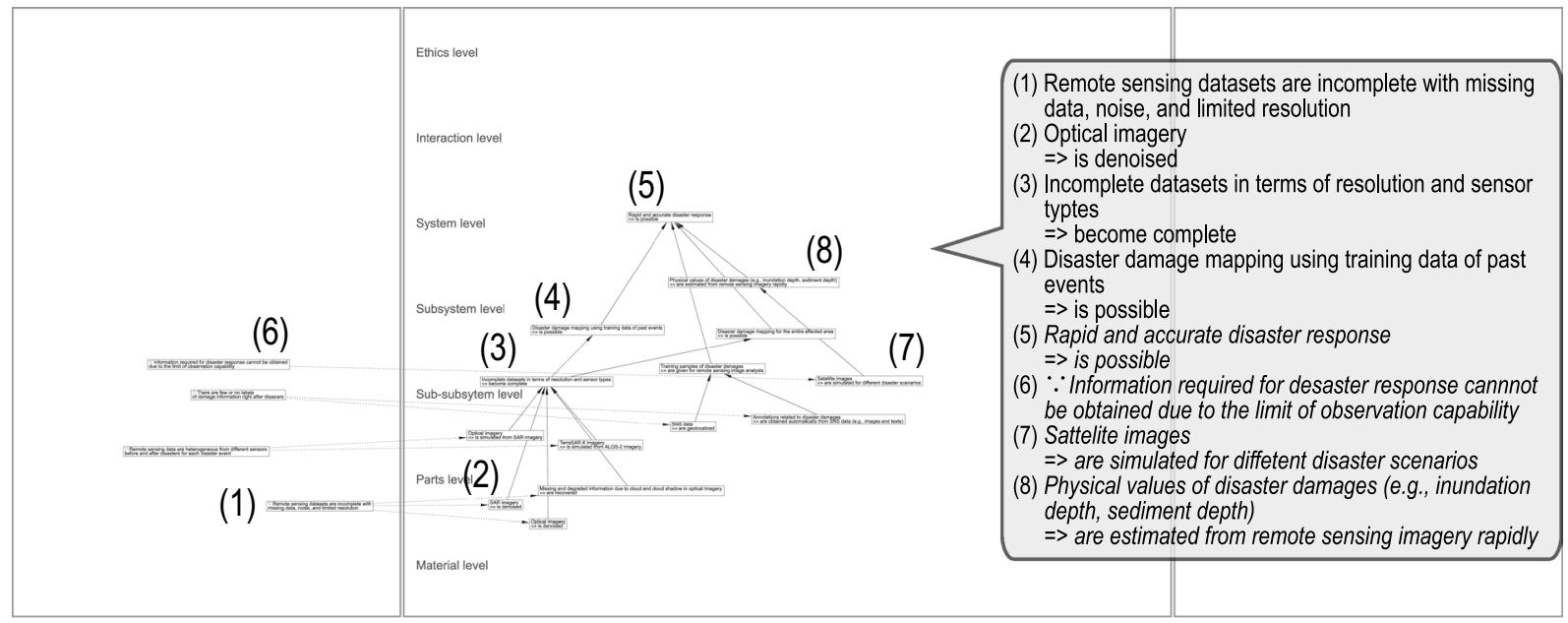

This subject edited own path without using the semi-automatic generation of items.

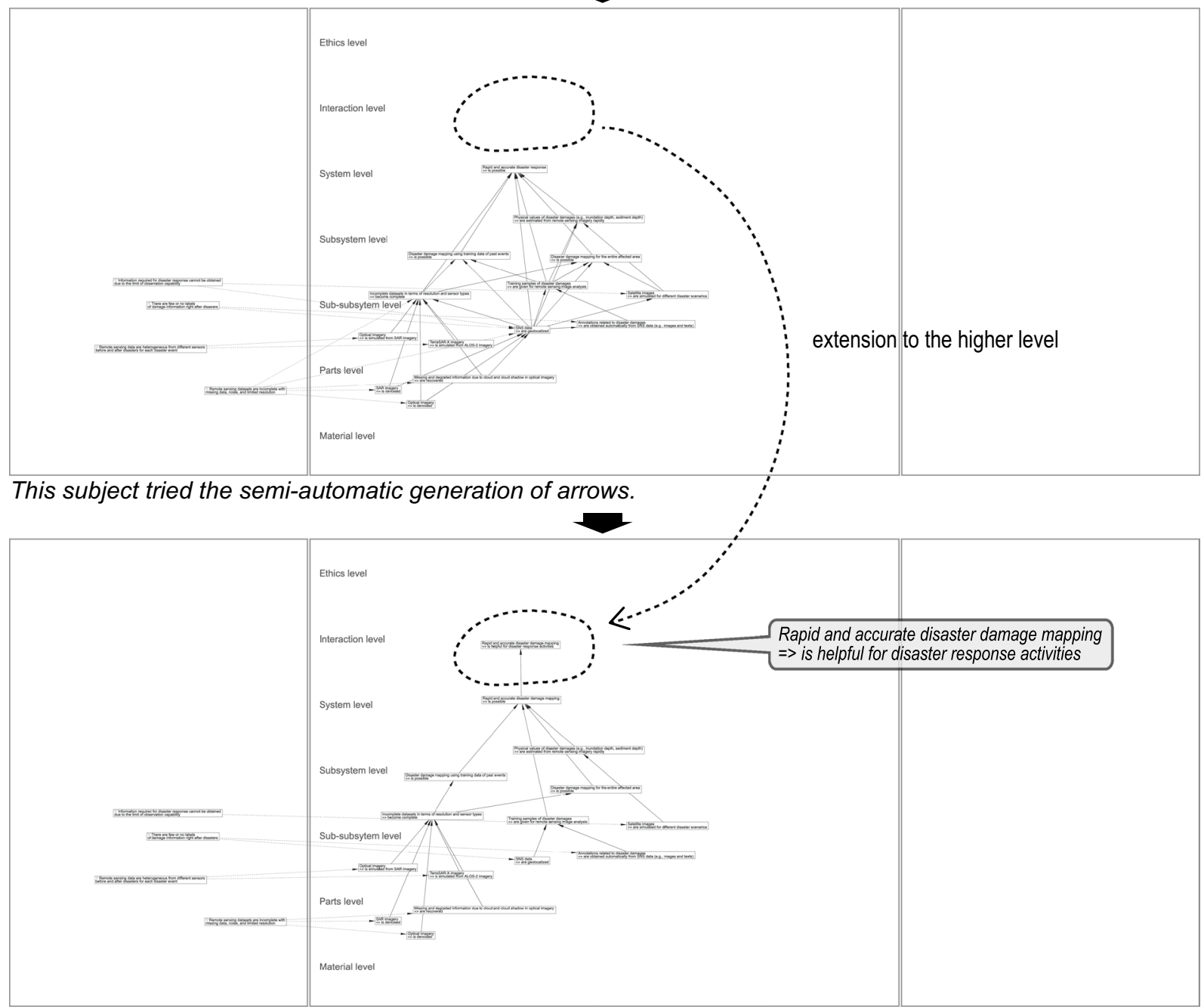

This subject canceled the result of the semi-automatic generation of arrows. However, he extend his tree upward by himself because he considered that the upward expression might be necessary.

Fig. 21 Outline of the editorial process of subject M (1) 


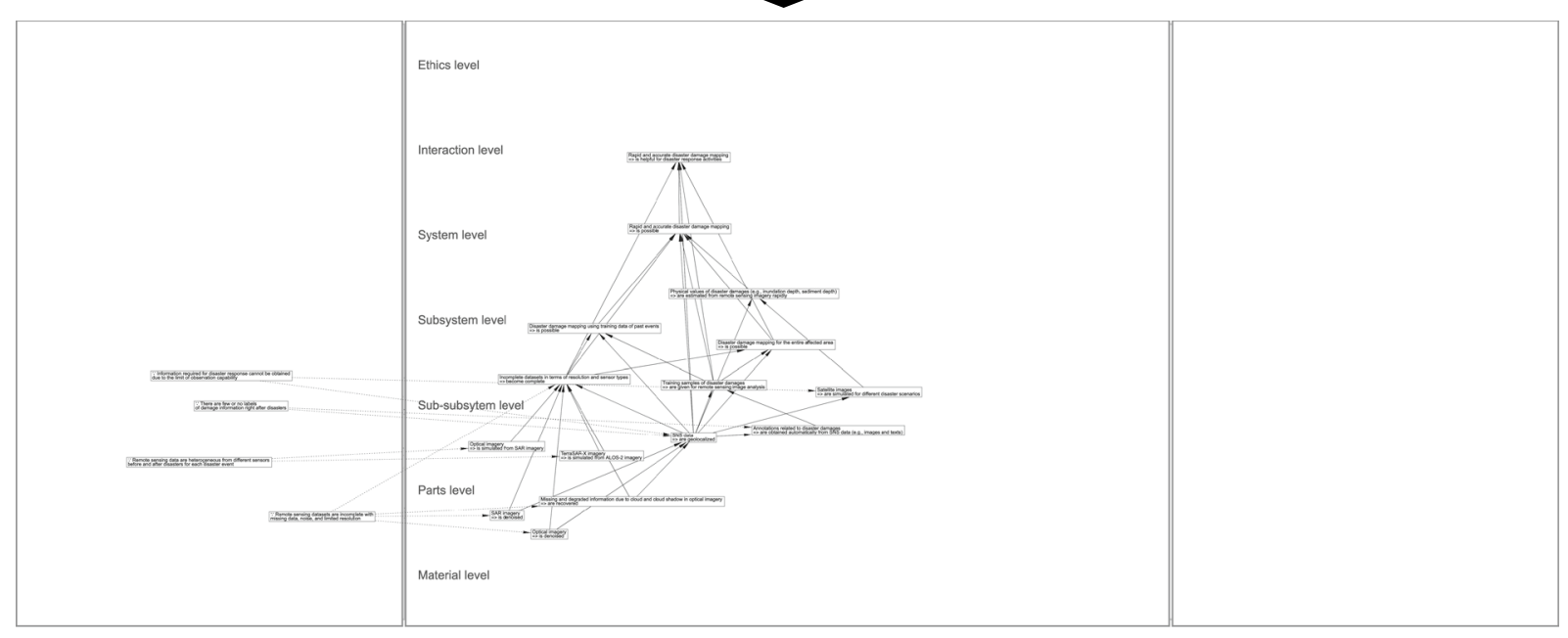

This subject tried the semi-automatic generation of arrows again in the hearing and perform further consideration.

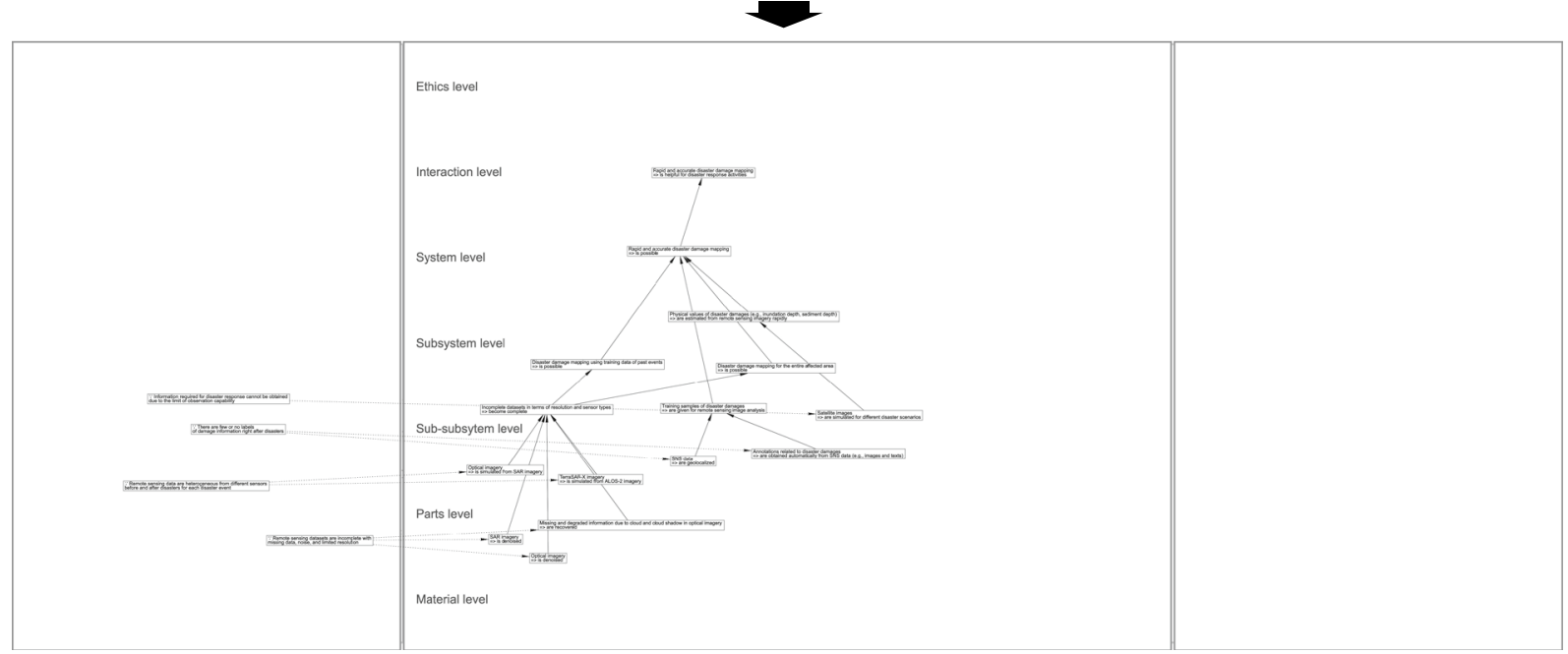

This subject canceled the result of the semi-automatic generation of arrows and finished the task.

Fig. 22 Outline of the editorial process of subject M (2) 


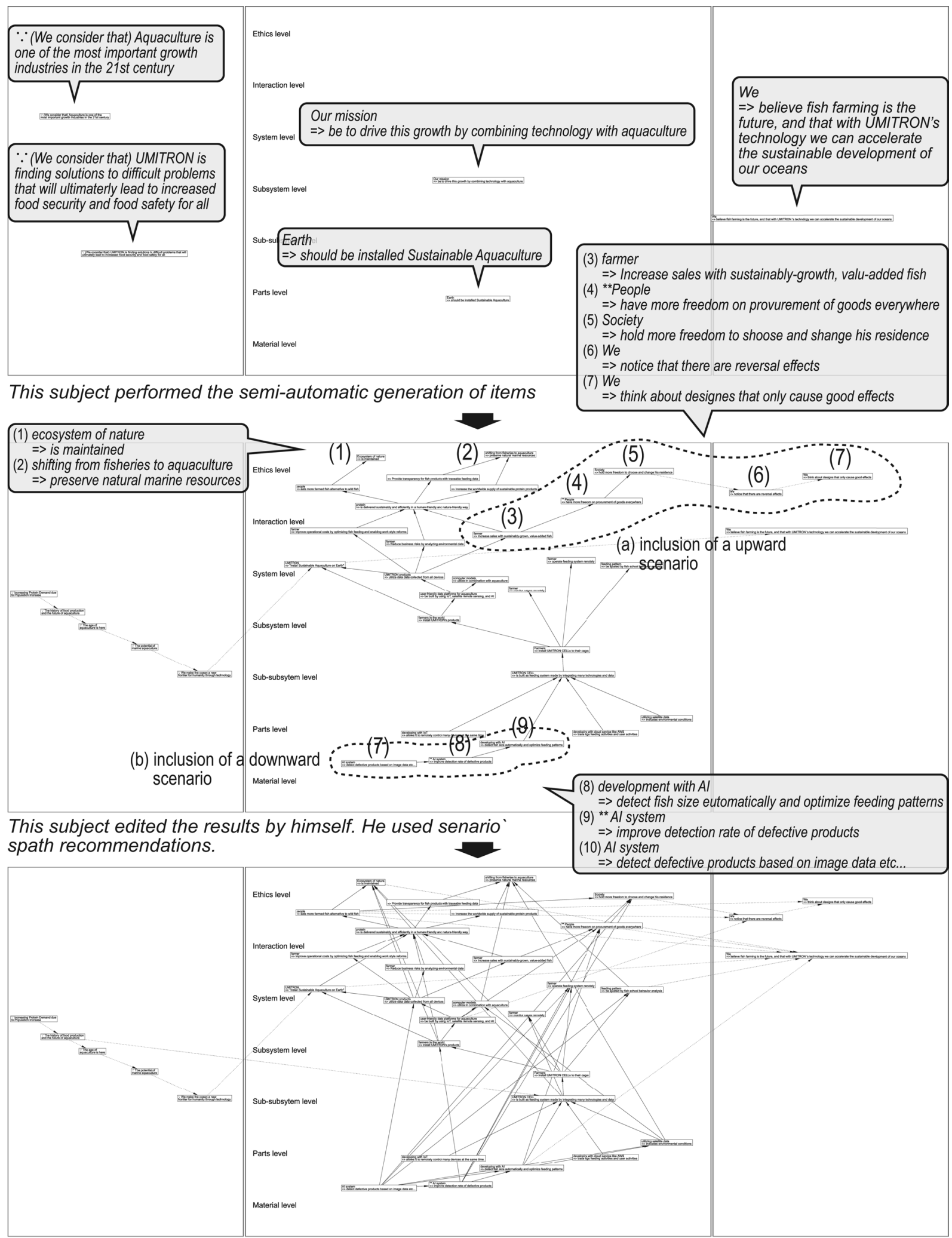

This subject tried the semi-automatic generation of arrows.

Fig. 23 Outline of the editorial process of subject J (1) [input text for semi-automatic generation of items was from UMITRON K. K. (2019)] 


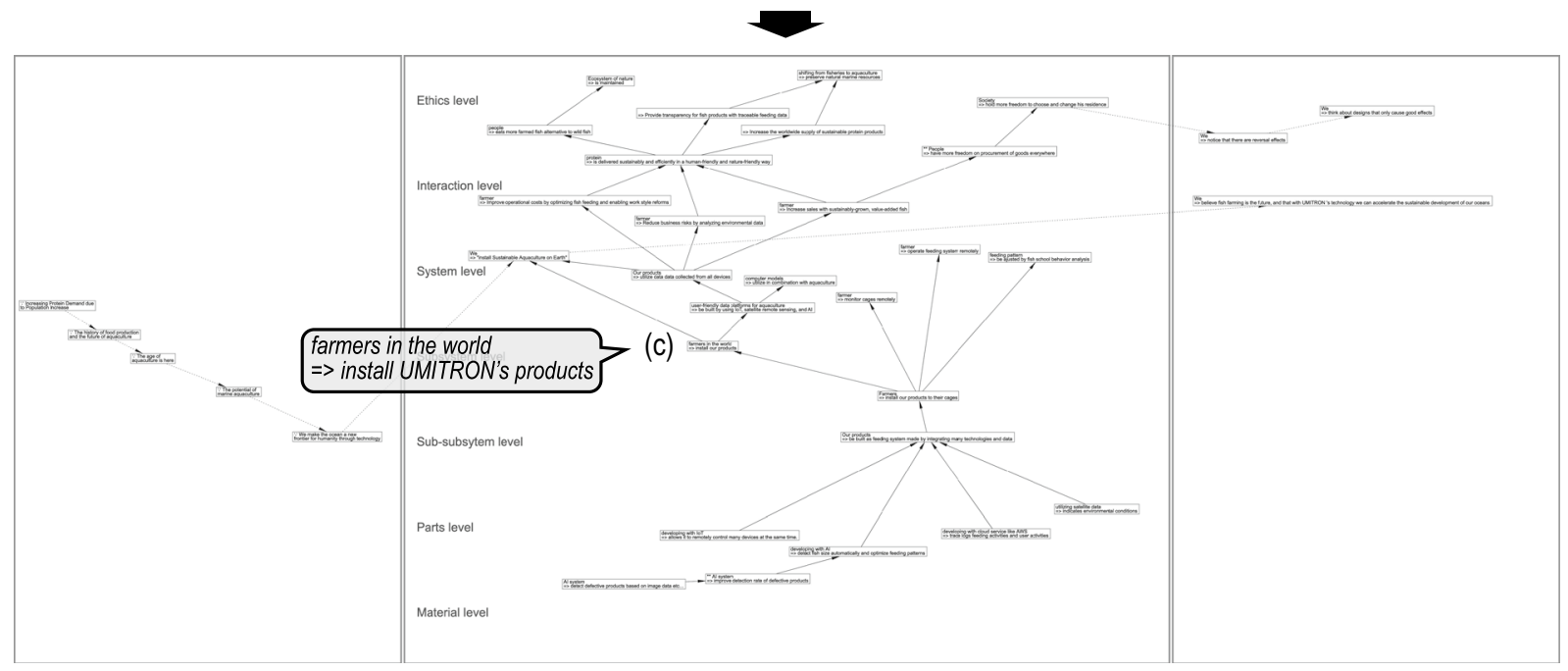

This subject canceled the result of the semi-automatic generation of arrows and finished the task.

Fig. 24 Outline of the editorial process of subject J (2) [input text for semi-automatic generation of items was from UMITRON K. K. (2019)] 


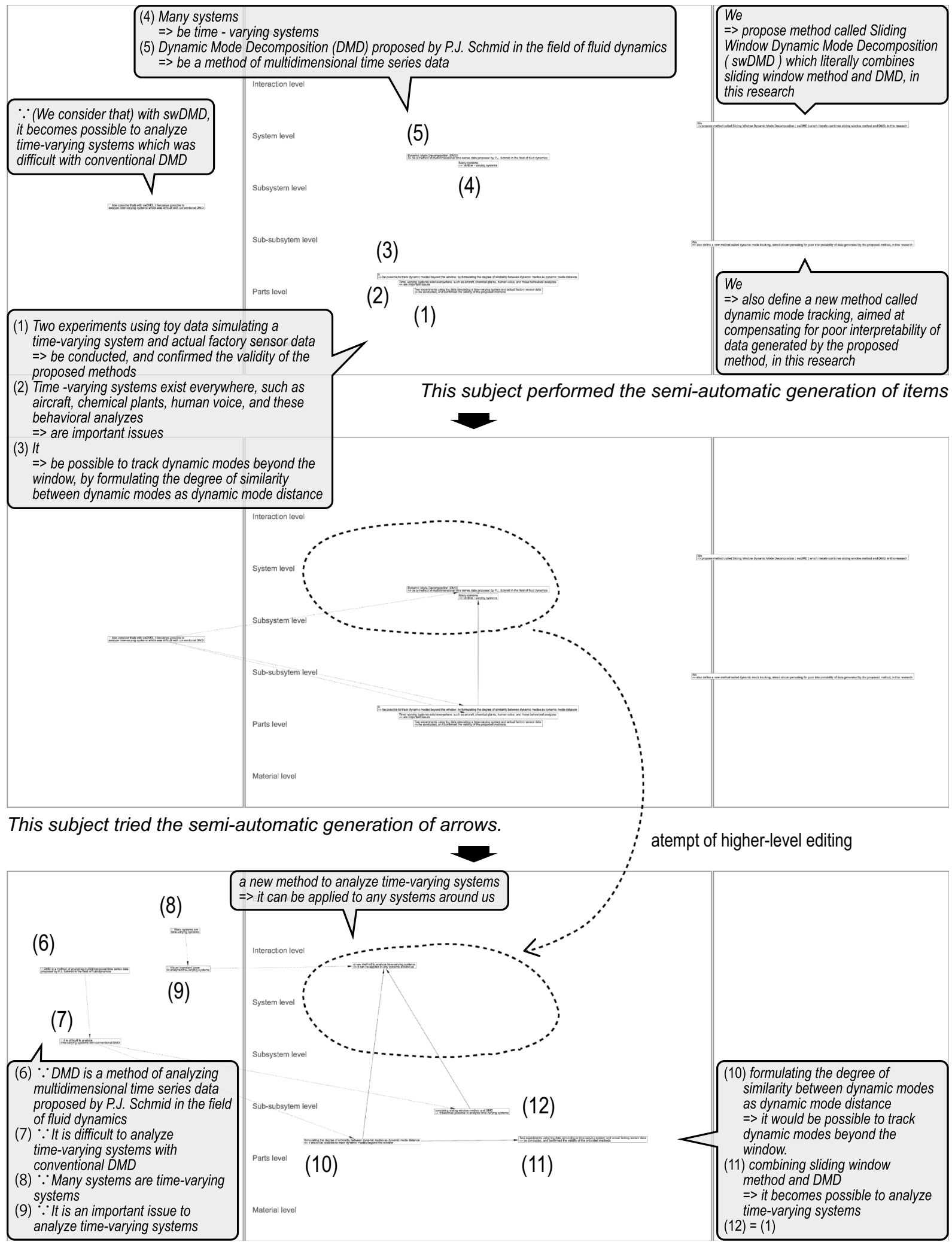

This subject edited the result of the semi-automatic generation of arrows and finished the task.

Fig. 25 Outline of the editorial process of subject F [input text for semi-automatic generation of items was from Minoda et al. (2019)] 


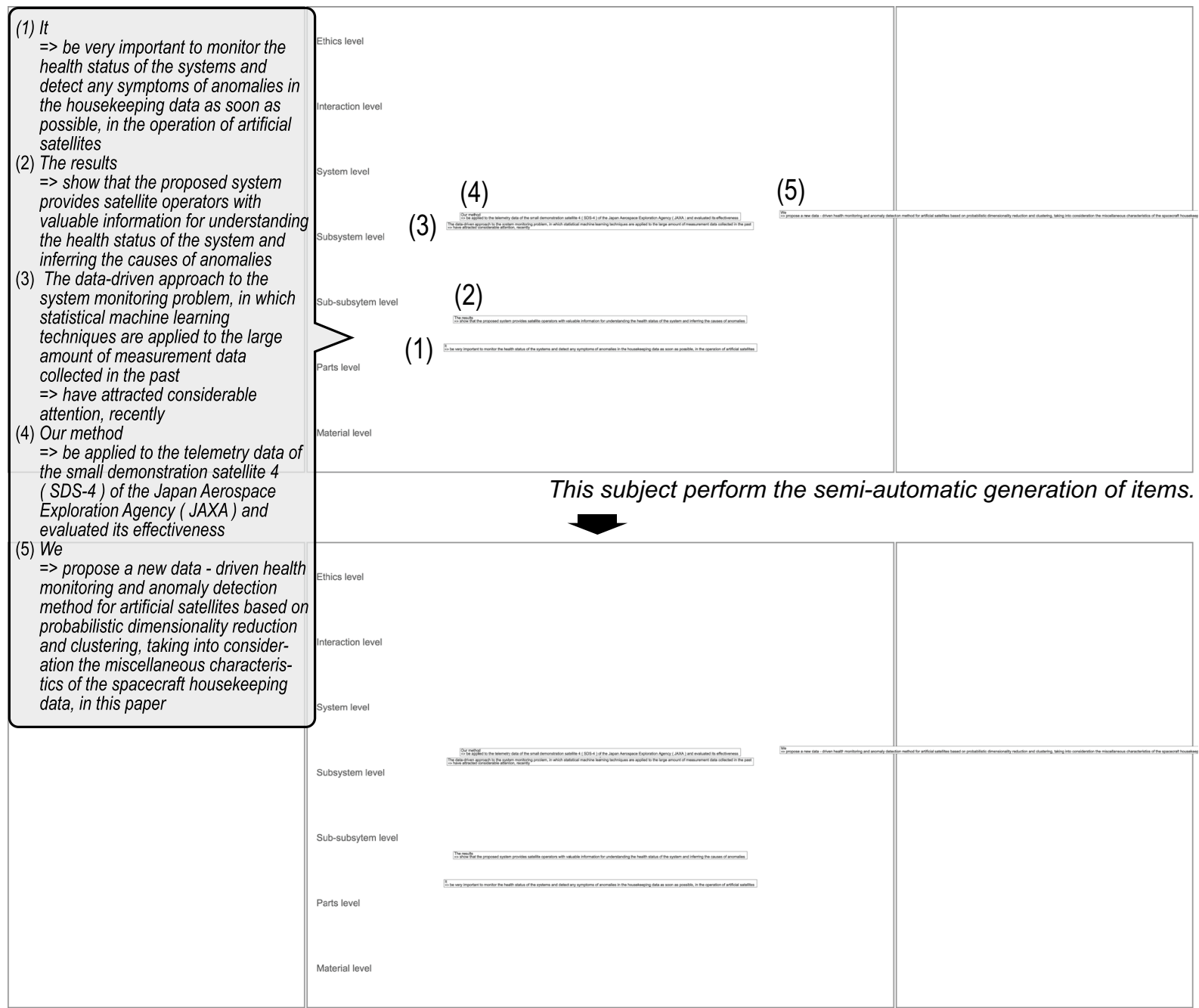

This subject tried the semi-automatic generation of arrows.

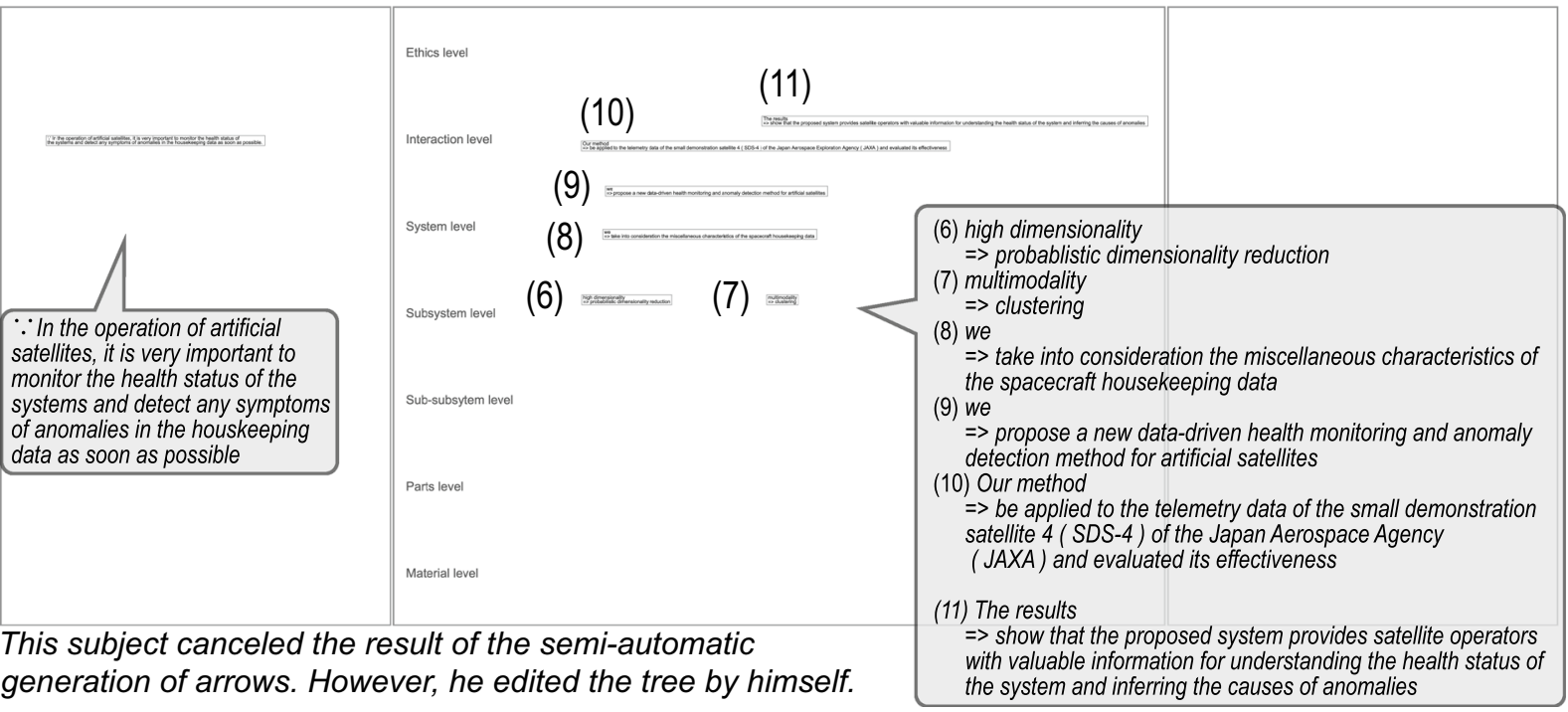

Fig. 26 Outline of the editorial process of subject K (1) [input text for semi-automatic generation of items was from Takehisa et al. (2017)] 


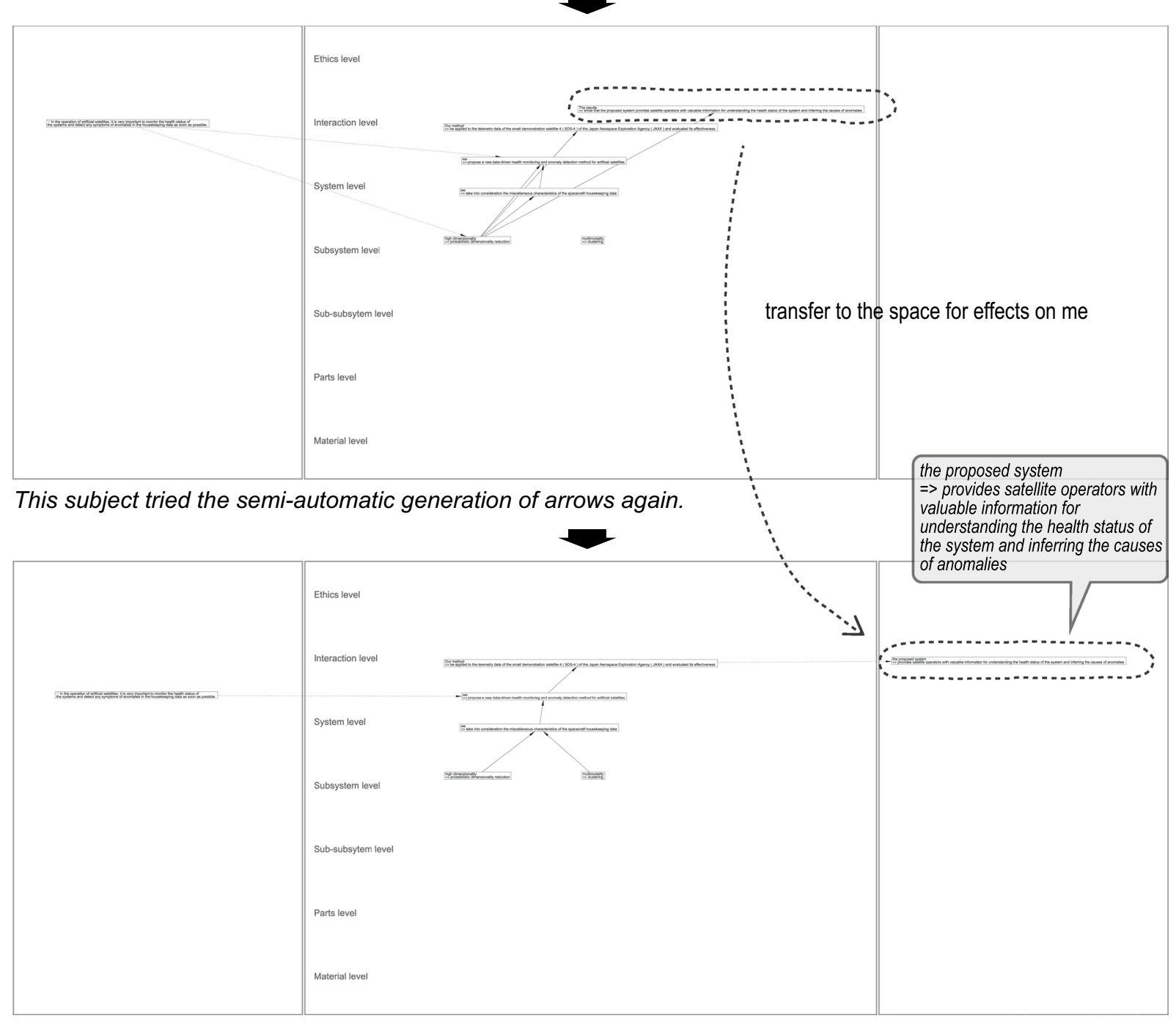

This subject re-edited the tree and finished the task.

Fig. 27 Outline of the editorial process of subject K (2) [input text for semi-automatic generation of items was from Takehisa et al. (2017)] 


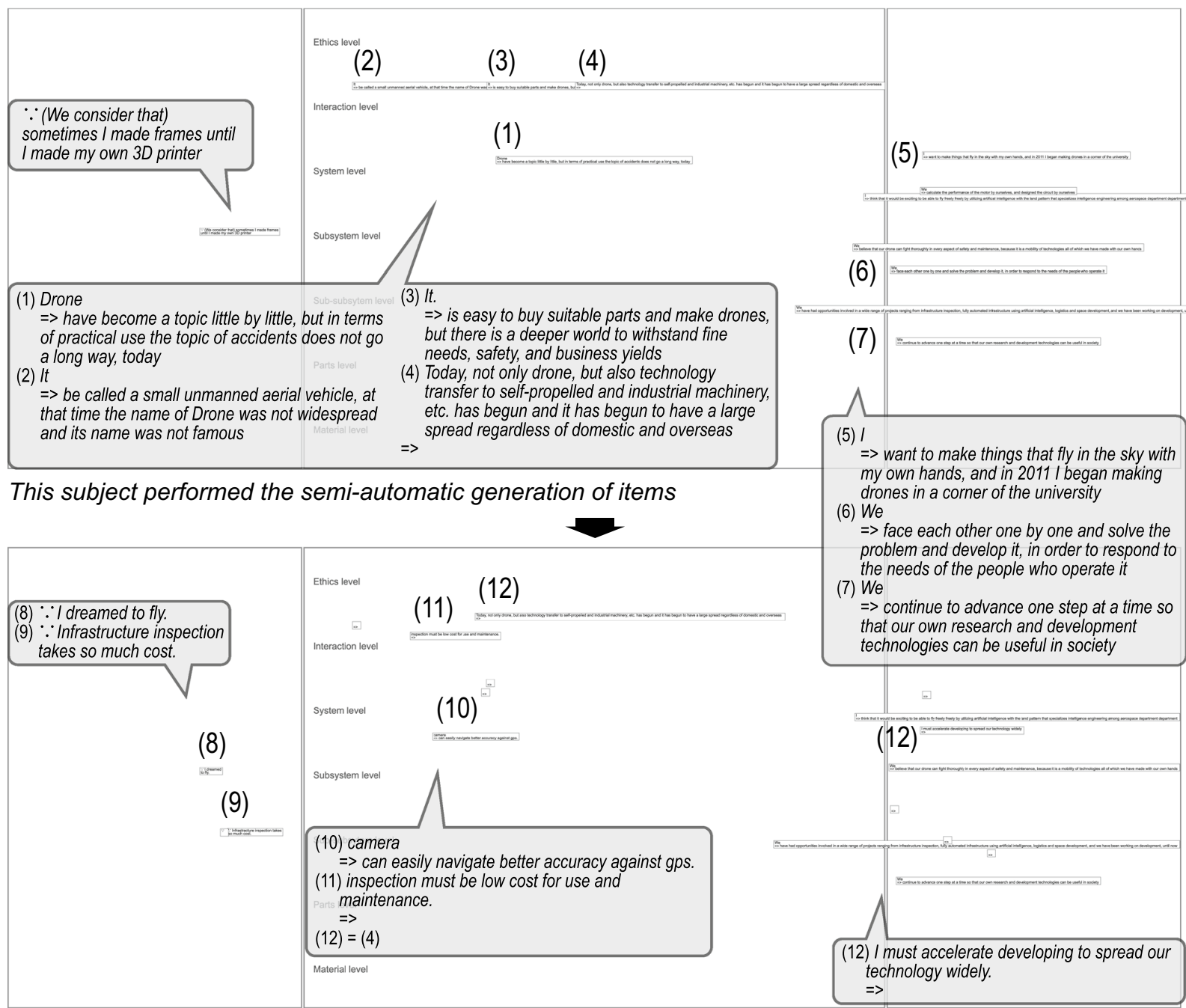

This subject edited the results by himself. He used senario path recommendations.

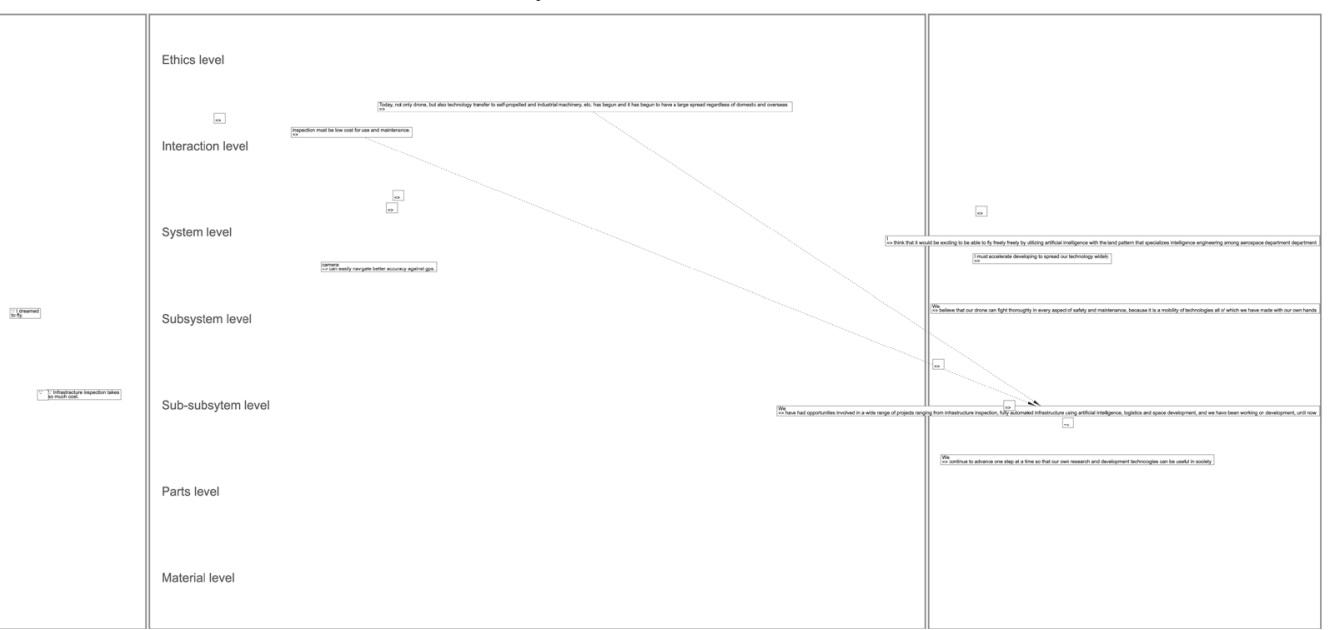

This subject tried the semi-automatic generation of arrows.

Fig. 28 Outline of the editorial process of subject L (1) [input text for semi-automatic generation of items was from Hongo Aerospace, Inc. (2019)] 


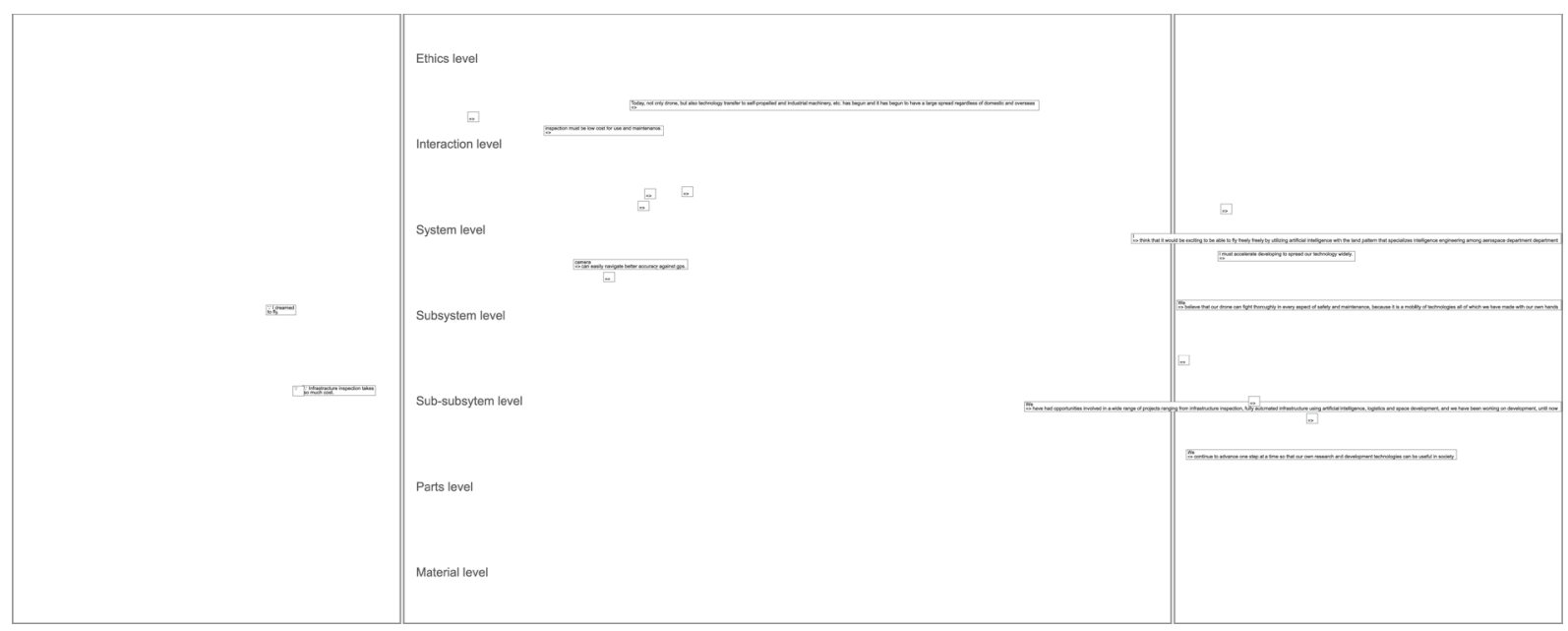

This subject canceled the result of the semi-automatic generation of arrows and finished the task.

Fig. 29 Outline of the editorial process of subject L (2) [input text for semi-automatic generation of items was from Hongo Aerospace, Inc. (2019)]

\section{References}

AIP (2019) Website of RIKEN Center for Advanced Intelligence Project (AIP). https://www.riken.jp/en/research/labs/aip/. Accessed Jan-Feb 2019

Boden M (1991) The creative mind: myths and mechanisms. Basic Books, New York

Ema A, Osawa H, Hattori H, Akiya N, Kanzaki N, Ichise R, Kukita M, Otani T, Kubo A, Komatani K, Saijo R, Tanaka M, Honda K, Miyano N, Yashiro Y, Yoshizawa G (2017) Breaking down silos: involving various researchers for driving hci research. In: CHI 2017 extended abstracts - proceedings of the 2017 ACM sigchi conference on human factors in computing systems, vol Part F127655. Association for Computing Machinery, pp 837-847. https://doi.org/10.1145/3027063.3052757

Finke RA, Ward TB, Smith SM (1992) Creative cognition. MIT Press, Cambridge

Fischer G, Nakakoji K, Ostwald JL, Stahl G, Sumner T (1993) Embedding critics in design environments. Knowl Eng Rev 8:285-307

Freedman B, Kahn PH Jr, Boring A (2006) Value sensitive design and information systems. In: Zhang P, Galletta DF (eds) Human-computer interaction and management information systems: foundations. M. E. Sharpe, New York, pp 349-372

Future of Life Institute (2017) Asilomar AI principles. https://www. futureoflife.org/ai-principles/

Hasida K (2014) Personal life repository as a distributed PDS and its dissemination strategy for healthcare services, In: AAAI spring symposium series, 2014

Kelly T (2001) The art of innovation: lessons in creativity from IDEO, America's leading design firm, Doubleday (with Jonathan Littman)

Krizhevsky A, Sutskever I, Hinton GE (2012) Imagenet classification with deep convolutional neural networks. In: Pereira F, Burges CJC, Bottou L, Weinberger KQ (eds) Advances in neural information processing systems 25. Curran Associates, Inc., Red Hook, pp 1097-1105

Le Q, Mikolov T (2014) Distributed representations of sentences and documents. In: Proceedings of the 31 st international conference on machine learning, JMLR.org, ICML'14, vol 32, pp II-1188-II-1196

Mikami K (2019) Koichi Mikami’s webpage (studies in progress...). https://www.studiesinprogress.wordpress.com/projects/projectdel ta/. Accessed Sep 2019

Miller J, Friedman B, Jancke G, Gill B (2007) Value tensions in design: The value sensitive design, development, and appropriation of a corporation's groupware system. In: Proceedings of the 2007 international ACM conference on supporting group work, GROUP '07 , Sanibel Island, Florida, pp 281-290

Minoda K, Yairi T, Hori K (2019) Data-driven health monitoring of high dimensional time-varying systems by tracking dynamic modes. In: Asia Pacific conference of the Prognostics and Health Management Society (PHMAP), Beijing, China

Murakami YP (2017) Science, past, present and future. In: From the early universe to the evolution of life, Japan-Germany roundtable

Nakakoji K (2007) From interfaces to interactions: an overview of SIGHI (1001 SIG nights). IPSJ Mag 48(2):202-203 (in Japanese)

Nickel M, Murphy K, Tresp V, Gabrilovich E (2016) A review of relational machine learning for knowledge graphs. Proc IEEE. https ://doi.org/10.1109/JPROC.2015.2483592

Oka T, Morimoto M (2015) An extraction and recognition method for partially hidden objects. In: 2015 international conference on informatics, electronics vision, ICIEV, pp 1-4. https://doi. org/10.1109/ICIEV.2015.7334055

Oka T, Morimoto M (2016) A recognition method for partially overlapped objects. In: 2016 World automation congress, WAC2016, pp 1-4. https://doi.org/10.1109/WAC.2016.7583005

Schinzinger R, Martin MW (2000) Introduction to engineering ethics. McGraw-Hill, New York

Schmid PJ (2010) Dynamic mode decomposition of numerical and experimental data. J Fluid Mech 656:5-28. https://doi. org/10.1017/s0022112010001217

Sekiguchi K, Hori K (2018a) Organic and dynamic tool for use with knowledge base of AI ethics for promoting engineers' practice of ethical AI design. AI Soc. https://doi.org/10.1007/s0014 6-018-0867-z 
Sekiguchi K, Hori K (2018b) Realization of organic and dynamic creativity support tool for promoting ethical AI design. In: Proceedings of the 13th international conference on knowledge, information and creativity support systems, KICSS2018

Sekiguchi K, Hori K (2019) Can ethics enhance creative design activity? Proc Des Soc Int Conf Eng Des 1(1):3181-3190. https://doi. org/10.1017/dsi.2019.325

Sekiguchi K, Tanaka K, Hori K (2010) "Design with discourse" to design from the "ethics level". In: Družovec TW, Jaakkola H, Kiyoki Y, Tokuda T, Yoshida N (eds) Volume 206: information modelling and knowledge bases XXI: frontiers in artificial intelligence and applications. IOS Press, pp 307-314. https://doi. org/10.3233/978-1-60750-477-1-307

Sekiguchi K, Terada M, Hori K, Nakagawa H (2018) Legal design incorporating risk mitigation measure of drone swarm design, special interest group on electronic intellectual property (IPSJEIP), IPSJ SIG technical report No 3:1-7 (in Japanese)

Simon HA (1996) The sciences of the artificial, 3rd edn. MIT Press, Cambridge

Spiekermann S (2016) Ethical IT innovation: a value-based system design approach. CRC Press, Boca Raton, p 220

Springwise.com (2016) New shopping baskets for just browsing or help wanted. https://www.springwise.com/new-shopping-baske ts-just-browsing-help-wanted/

Takehisa Y, Naoya T, Tetsuo O, Yuta N, Naoki N, Noboru T (2017) A data-driven health monitoring method for satellite housekeeping data based on probabilistic clustering and dimensionality reduction. IEEE Trans Aerosp Electron Syst. https://doi.org/10.1109/ TAES.2017.2671247

Terada M (2018) Legal study on drone highway. Inf Netw Law Rev 16:31-49 (in Japanese)

The Conference toward AI Network Society (2017a) Draft AI R\&D guidelines for international discussions (tentative translation) . http://www.soumu.go.jp/main_sosik/joho_tsusin/eng/Relea ses/Telecommunications/170728_05.html

The Conference toward AI Network Society (2017b) Report 2017: toward promotion of international discussions on AI networking: overview. http://www.soumu.go.jp/main_sosik/joho_tsusin/eng/ Releases/Telecommunications/170728 05.html

The Conference toward AI Network Society (2017c) Socioeconomic impact of AI networking: Preliminary assessment. http://www. soumu.go.jp/menu_news/s-news/01iicp01_02000067.html, (attached paper 3 of Report 2017: toward promotion of international discussions on AI networking) (in Japanese)

The IEEE Global Initiative for Ethical Considerations in Artificial Intelligence and Autonomous Systems (2016) Ethically aligned design: a vision for prioritizing wellbeing with artificial intelligence and autonomous systems, version 1. http://www.standards. ieee.org/develop/indconn/ec/autonomous_systems.html

The IEEE Global Initiative on Ethics of Autonomous and Intelligent Systems (2017) Ethically aligned design: a vision for prioritizing wellbeing with artificial intelligence and autonomous systems, version 2. http://www.standards.ieee.org/develop/indconn/ec/ autonomous_systems.html

The IEEE Global Initiative on Ethics of Autonomous and Intelligent Systems (2019) Ethically aligned design: a vision for prioritizing human well-being with autonomous and intelligent systems, 1st edn. https://www.standards.ieee.org/content/ieee-standards/en/ industry-connections/ec/autonomous-systems.html

Umeda Y, Takeda H, Tomiyama T, Yoshikawa H (1990) Function, behaviour, and structure. In: Gero JS (ed) Volume 1: applications of artificial intelligence in engineering V. Springer and Computational Mechanics Publications, Berlin, pp 177-194

Umeda Y, Tomiyama T, Yoshikawa H (1997) Proposal of functionbehavior-state modeling to support functional design. J Jpn Soc Precis Eng 63:795-800. https://doi.org/10.2493/jjspe.63.795 (in Japanese)

van der Poel I (2015) Ethics and technology 2.0: responsible innovation and value sensitive design. In: Presentaties at Filosofie \& Techniek, KIVI

Verbeek PP (2011) Moralizing technology. Understanding and designing the morality of things. University of Chicago Press, Chicago

Wang K, Nickerson JV (2017) A literature review on individual creativity support systems. Comput Hum Behav 74:139-151. https://doi. org/10.1016/j.chb.2017.04.035

Website of Department of Aeronautics and Astronautics, the University of Tokyo. http://www.aerospace.t.u-tokyo.ac.jp/english/index .html. Accessed Feb 2019

Website of Hongo Aerospace, Inc. http://www.hongo-aerospace.com/ en.html. Accessed May-June 2019

Website of UMITRON K. K. (2019) (Mission page). https://www.umitr on.com/en/mission.html. Accessed Apr 2019

Whitbeck C (1998) Ethics in engineering practice and research. Cambridge University Press, Cambridge

Wittgenstein L (1969) On certainty. Basil Blackwell, Oxford, p 44 [first Harper Torchbook edition published 1972]

Wurms G (2016) Security and privacy in indoor positioning, infsoft blog. https://www.infsoft.com/blog/security-and-privacy-in-indoo r-positioning. Accessed Oct 2018

Yanmaz E, Yahyanejad S, Rinner B, Hellwagner H, Bettstetter C (2017) Drone networks: communications, coordination, and sensing. Ad Hoc Netw. https://doi.org/10.1016/j.adhoc.2017.09.001

Yoshikawa H (1979) Introduction to general design theory. J Jpn Soc Precis Eng 45(536):906-912. https://doi.org/10.2493/jjspe 1933.45.906 (in Japanese)

Yoshikawa H (1981) General theory of design process. J Jpn Soc Precis Eng 47(4):405-410 https://doi.org/10.2493/jjspe1933.47.405 (in Japanese)

Yoshikawa H (2012) Design methodology for research and development strategy: realizing a sustainable society. Center for Research and Development Strategy Japan Science and Technology Agency. https://www.jst.go.jp/crds/en/publications/CRDS-FY2010-XR25E.html

Publisher's Note Springer Nature remains neutral with regard to jurisdictional claims in published maps and institutional affiliations. 